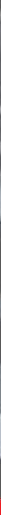

\author{
IntechOpen
}

\title{
Ontological Analyses in Science, Technology and Informatics
}

Edited by Andino Maseleno and Marini Othman

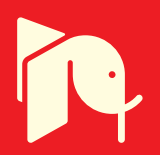





\section{Ontological Analyses in Science, Technology and Informatics}

Edited by Andino Maseleno and Marini Othman 

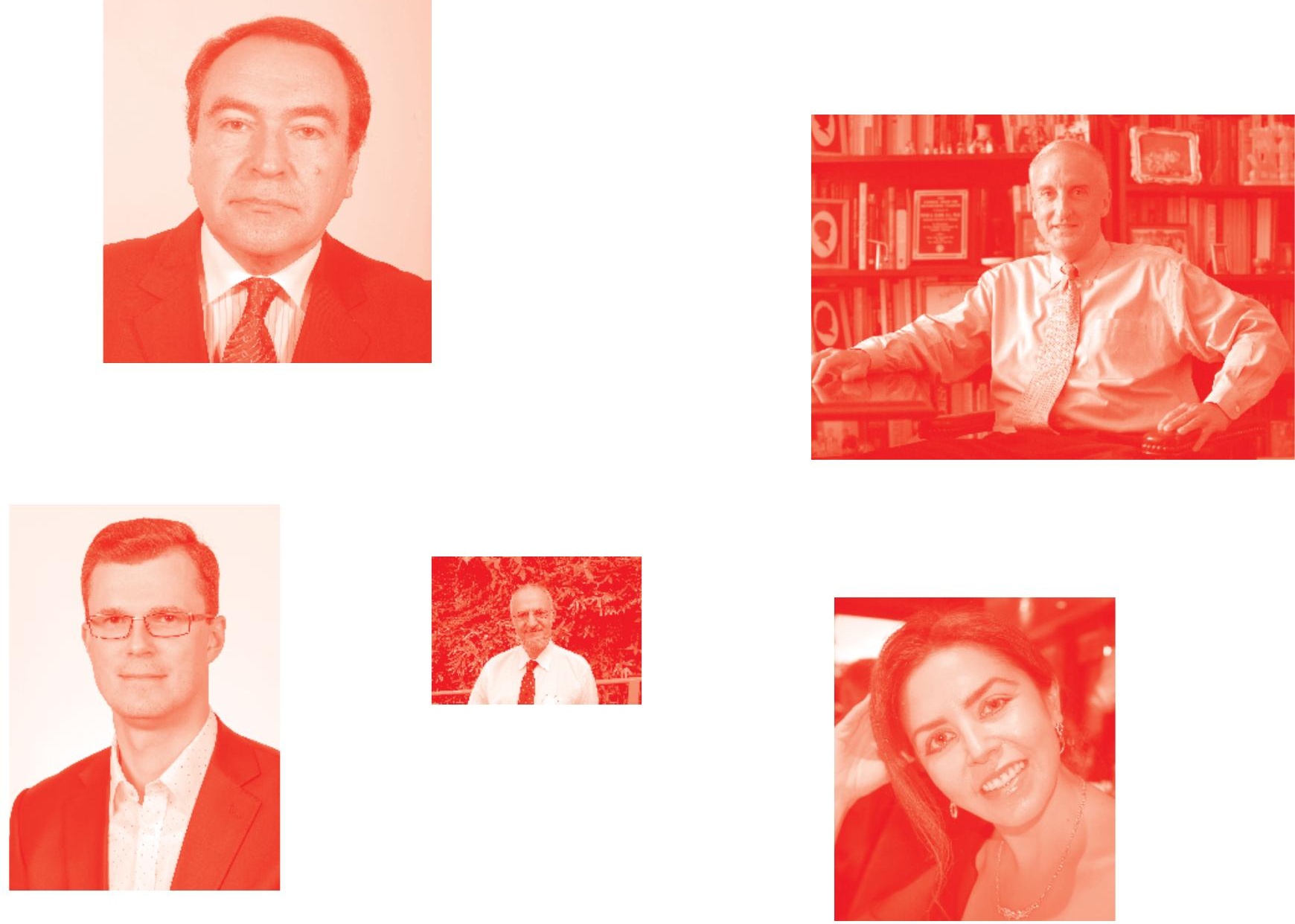

Supporting open minds since 2005
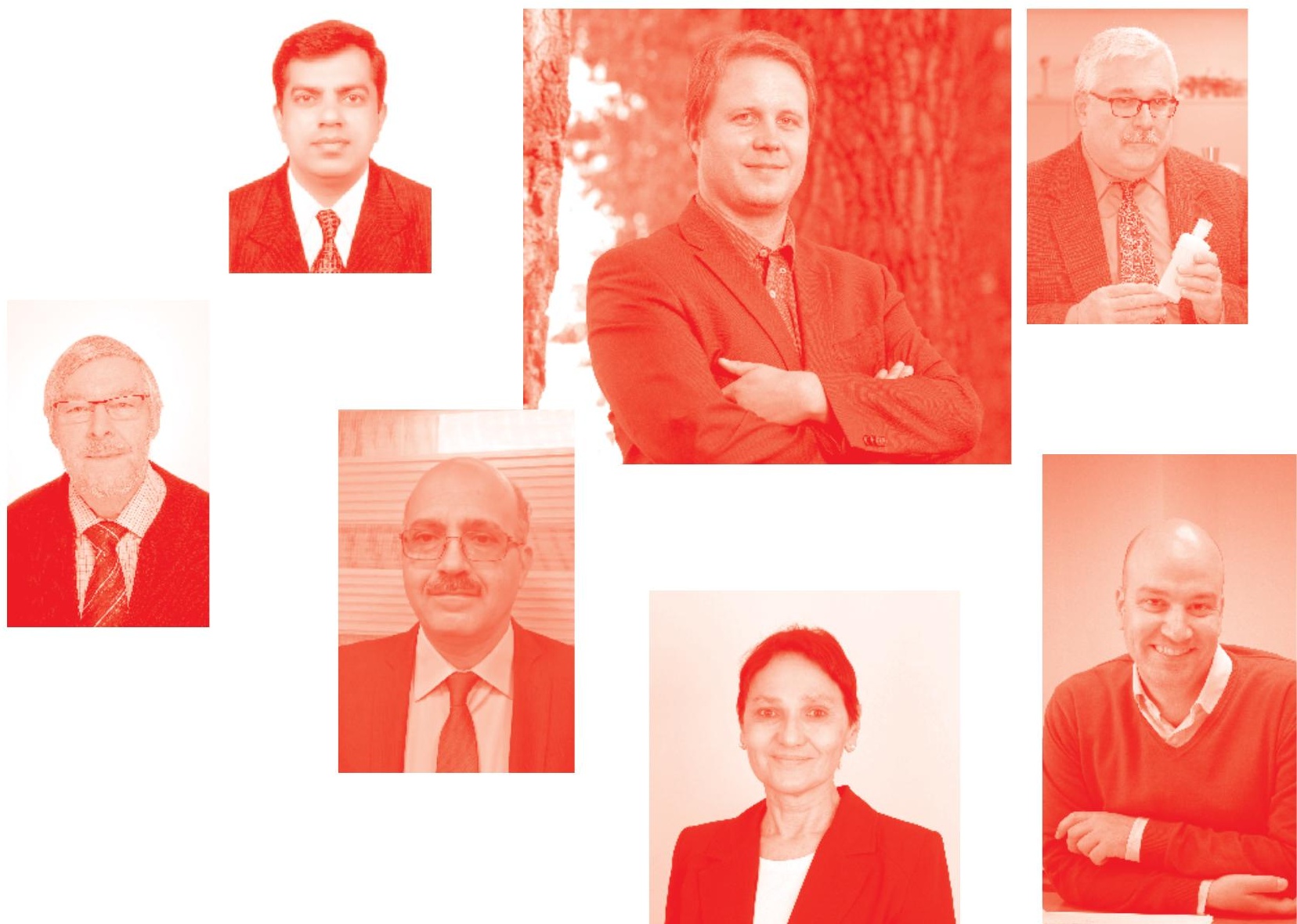
Ontological Analyses in Science, Technology and Informatics

http: //dx. doi.org/10.5772/intechopen . 77594

Edited by Andino Maseleno and Marini Othman

\section{Contributors}

Bohdan Hejna, Martin Žáček, Alena Lukasová, Marek Vajgl, Petr Raunigr, Sukumar Mandal, Friska Natalia, Dea Cheria, Santi Surya, JinTa Weng, Jing Qiu, Ying Gao

( ) The Editor(s) and the Author(s) 2020

The rights of the editor(s) and the author(s) have been asserted in accordance with the Copyright, Designs and Patents Act 1988. All rights to the book as a whole are reserved by INTECHOPEN LIMITED. The book as a whole (compilation) cannot be reproduced, distributed or used for commercial or non-commercial purposes without INTECHOPEN LIMITED's written permission. Enquiries concerning the use of the book should be directed to INTECHOPEN LIMITED rights and permissions department (permissions@intechopen.com).

Violations are liable to prosecution under the governing Copyright Law .

\section{(cc) BY}

Individual chapters of this publication are distributed under the terms of the Creative Commons Attribution 3.0 Unported License which permits commercial use, distribution and reproduction of the individual chapters, provided the original author(s) and source publication are appropriately acknowledged. If so indicated, certain images may not be included under the Creative Commons license. In such cases users will need to obtain permission from the license holder to reproduce the material. More details and guidelines concerning content reuse and adaptation can be found at http : //www . intechopen . com/copyright-policy . html .

\section{Notice}

Statements and opinions expressed in the chapters are these of the individual contributors and not necessarily those of the editors or publisher. No responsibility is accepted for the accuracy of information contained in the published chapters. The publisher assumes no responsibility for any damage or injury to persons or property arising out of the use of any materials, instructions, methods or ideas contained in the book.

First published in London, United Kingdom, 2020 by IntechOpen IntechOpen is the global imprint of INTECHOPEN LIMITED, registered in England and Wales, registration number: 11086078 , 7th floor, 10 Lower Thames Street, London,

EC3R 6AF, United Kingdom

Printed in Croatia

British Library Cataloguing-in-Publication Data

A catalogue record for this book is available from the British Library

Additional hard and PDF copies can be obtained from orders@intechopen.com

Ontological Analyses in Science, Technology and Informatics

Edited by Andino Maseleno and Marini Othman

p. $\mathrm{cm}$.

Print ISBN 978-1-78985-547-Ø

Online ISBN 978-1-78985-548-7

eBook (PDF) ISBN 978-1-83881-092-4 


\section{We are IntechOpen, \\ the world's leading publisher of Open Access books}

\section{Built by scientists, for scientists}

\section{$4,900+$}

Open access books available

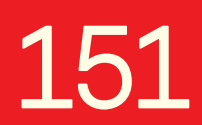

Countries delivered to

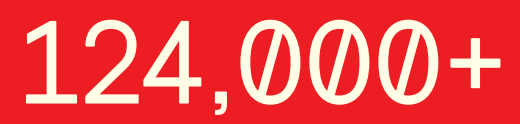

International authors and editors

Our authors are among the

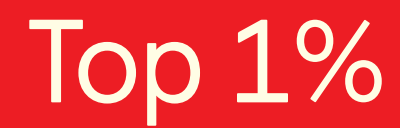

most cited scientists

Contributors from top 500 universities
$140 \mathrm{M}+$

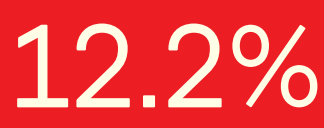

$12.2 \%$

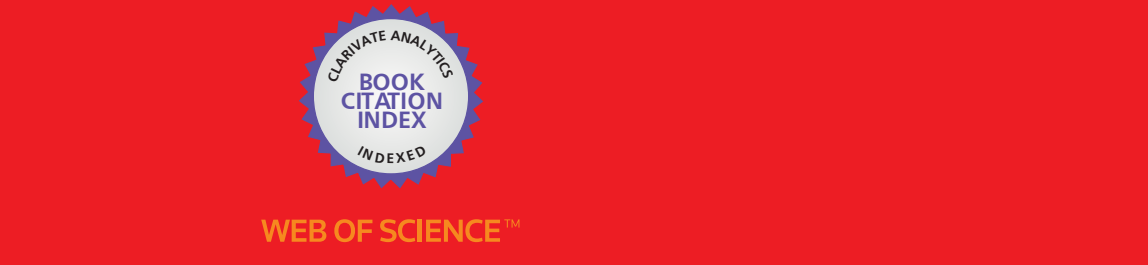

Selection of our books indexed in the Book Citation Index

in Web of Science ${ }^{\mathrm{TM}}$ Core Collection (BKCI)

\section{Interested in publishing with us? \\ Contact book.department@intechopen.com}

Numbers displayed above are based on latest data collected.

For more information visit www.intechopen.com 



\section{Meet the editors}

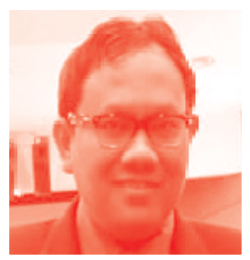

Dr Andino Maseleno is a lecturer at STMIK Pringsewu, Lampung, Indonesia. He was a postdoctoral researcher at the Institute of Informatics and Computing Energy, Universiti Tenaga Nasional, Malaysia, from 2018 to 2019. He was a visiting fellow at the Centre for Lifelong Learning, Universiti Brunei Darussalam, Brunei, Darussalam, from July 2016 to March 2017. He received his BSc degree in Informatics Engineering from UPN

Veteran Yogyakarta, Indonesia in 2005, his M.Eng. in Electrical Engineering from Gadjah Mada University, Indonesia in 2009, and his Ph.D. in Computer Science from Universiti Brunei Darussalam, Brunei Darussalam in 2015.

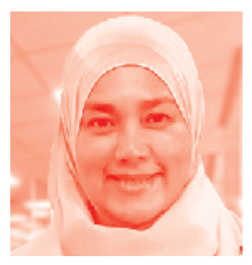

Professor Marini Othman is the director of the Institute of Informatics and Computing Energy, Universiti Tenaga Nasional, Malaysia. She received her BSc in Computer Science from Indiana State University, USA, in 1986, her MSc in Computer Science from Western Kentucky University, USA, in 1987, and her PhD in Industrial Computing from Universiti Kebangsaan Malaysia, Malaysia, in 2010. 



\section{Contents}

$\begin{array}{lll}\text { Preface } & \text { XIII }\end{array}$

Chapter $1 \quad 1$

Common Gnoseological Meaning of Gödel and Caratheodory Theorems by Bohdan Hejna

Chapter 2

Knowledge Patterns within the Conception of Semantic Web

by Martin Žáček, Alena Lukasová, Marek Vajgl and Petr Raunigr

Chapter 3

Ontology Language XOL Used for Cross-Application Communication by Jinta Weng, Jing Qiu and Ying Gao

Chapter 4

An Ontology-Based Approach to Diagnosis and Classification for an Expert System in Health and Food

by Friska Natalia, Dea Cheria and Santi Surya

Chapter 5

Taxonomy and Ontology Management Tools: A General Explanation by Sukumar Mandal 



\section{Preface}

Ontology in the philosophical sense is concerned with the nature of being as well as with respective basic concepts. Aristotle called ontology the first philosophy. Despite its metaphysical nature, this first philosophy or ontology is a significant attempt to introduce a systematic approach to the process of thinking about the world surrounding us, about our conceptual thinking and about us ourselves.

Today's advanced science inherited the original ontology's efforts to systemize and conceptualize. In this respect the concept of ontology nowadays is a non-speculative methodology for studying reality objects and used tools, both of which are important for our orientation in the physical, technical, mental, and social worlds. As such, we deal with the process of studying, as well as with the outcomes of such study, of the objects observed or created by man and their respective concepts, relations between them, and relations between their systems in different fields of science.

Understood in this modern and precise way, this book applies the meaning of ontology in a search for semantic meanings of objects and concepts. It examines the relations between general or abstract terms and their meanings using a variety of logics such as automated indexation of databases. Therefore, the meaning of "to be" and "how to be" is exercised in complex or abstracted cases, for example, in number theory. The objective is to model, categorize, and conceptualize the knowledge available, and to design relevant schemes such as function-related diagrams or tree structures. Then, pending on the field of study, it is possible to discuss, introduce, and compare a variety of ontologies as the formal models representing our knowledge of the field or the problem. As such, it is possible to discuss ontology as it relates to experimental organization, software and systems engineering, artificial intelligence, the Semantic Web, and informatics and information sciences.

Through these kinds of ontologies we understand the models with standard structures of entities, classes, qualities, and relations. Such models are explicit, created using suitable language and formalized descriptions of the given systems of the objects or their respective concepts and relations. Then we speak about a model or a data model of the problems. The language used for these purposes can be formal (e.g., languages of physical or mathematical theories), semiformal, or informal, especially in the initial stages of studying the problems.

However, ontologies are not only the final formal and declarative representations, models, or data models of given problems but they are also the methodology and, consequently, the method and the process of creating these declarations or models. Ontology as a process creates, uses, and provides, as its output, a model or descriptive ontology. Such a model is a glossary of definitions of concepts corresponding to the objects, a thesaurus of definitions of the relations among the concepts and the respective objects. Thus, the ontology in this descriptive sense is both vocabulary and grammar that are used to keep and pass over the knowledge of the problems studied. 
This book Ontological Analyses in Science, Technology and Informatics is the illustration of the modern and scientific application of ontology.

Chapter 1 deals with logic inference ontology used in the theory of proof based upon the language of physics; Chapter 2 examines knowledge-based pattern ontology in the Resource Description Framework (RDF) language in Semantic Web applications; Chapter 3 deals with implementation of the ontological XOL language in cross-application communication; Chapter 4 discusses ontological studies in the fields of diagnosis and expert systems in health and food; and Chapter 5 covers management ontology and taxonomy.

This book is designed for theorists as well as those persons dealing with designing, developing, managing, and decision-making in the field of ontology.

I would like to thank the publishing process managers and technical staff at IntechOpen for their commitment, friendly effort, and support throughout the production of this book. Finally, I express my thanks to all the contributing authors for their valuable chapters.

Ing. Bohdan Hejna, Ph.D., dr. h. c.

Department of Mathematics, University of Chemistry and Technology, Prague, Czech Republic 


\title{
Common Gnoseological Meaning of Gödel and Caratheodory Theorems
}

\author{
Bohdan Hejna
}

\begin{abstract}
We will demonstrate that the I. and the II. Caratheodory theorems and their common formulation as the II. Law of Thermodynamics are physically analogous with the real sense of the Gödel's wording of his I. and II. incompleteness theorems. By using physical terms of the adiabatic changes the Caratheodory theorems express the properties of the Peano Arithmetic inferential process (and even properties of any deductive and recursively axiomatic inference generally); as such, they set the physical and then logical limits of any real inference (of the sound, not paradoxical thinking), which can run only on a physical/thermodynamic basis having been compared with, or translated into the formulations of the Gödel's proof, they represent the first historical and clear statement of gnoseological limitations of the deductive and recursively axiomatic inference and sound thinking generally. We show that semantically understood and with the language of logic and metaarithmetics, the full meaning of the Gödel proof expresses the universal validity of the $I I$. law of thermodynamics and that the Peano arithmetics is not self-referential and is consistent. ${ }^{1}$
\end{abstract}

Keywords: arithmetic formula, thermodynamic state, adiabatic change, inference

\section{Introduction}

To show that the real/physical sense of the Gödel incompleteness theoremsthat the very real sense of them-is the meta-arithmetic-logical analog of the Caratheodory's claims about the adiabatic system (that they are the analog of the sense of the II. Law of Thermodynamics), we compare the states in the state space of an adiabatic thermodynamic system with arithmetic formulas and the Peano inference is compared with the adiabatic changes within this state space. The whole set of the states now not achievable adiabatically represents the existence of the states on an adiabatic path, but this fact is not expressible adiabatically. This property of which is the

\footnotetext{
1 The reader of the paper should be familiar with the Gödel proof's way and terminology; SMALL CAPITALS in the whole text mean the Gödel numbers and working with them. This chapter is based, mainly, on the [1-4]. This paper is the continuation of the lecture Gödel Proof, Information Transfer and Thermodynamics [4].
} 
analog of the sense of Gödel undecidable formula. Nevertheless, any of these states, now not achievable adiabatically in the given state space (of the given adiabatic system), is achievable adiabatically but in the redefined and wider adiabatic system with its state space divided between adiabatic and not adiabatic parts again. These states (which are achievable only when the previous subsystem is part of the new actual system, both are consistent/adiabatic) represent arithmetic but not the Peano arithmetic formulas and also are bearing the property of their whole set. Also they can be axioms of the higher/superior inference including the previous one-the general arithmetic inference is further ruled by the same and repeated principle of widening the axiomatics and with same thermodynamic analogy using the redefined and widened new adiabatic system and its settings and with the same limitation by the impossibility to proof both the consistency of the given inferential system and, in our analogy, the adiabacity of its given adiabatic analog, by means of themselves. The consistency of the inferential system and adiabacity of its analog (and their abilities generally) are defined and proved by outer construction, outer limitations, and outer settings only (compare this our claim with the Gödel's claim for the Peano arithmetic inference “... in the Peano arithmetic system exists ...").

Caratheodory common formulation of the II. P.T.:

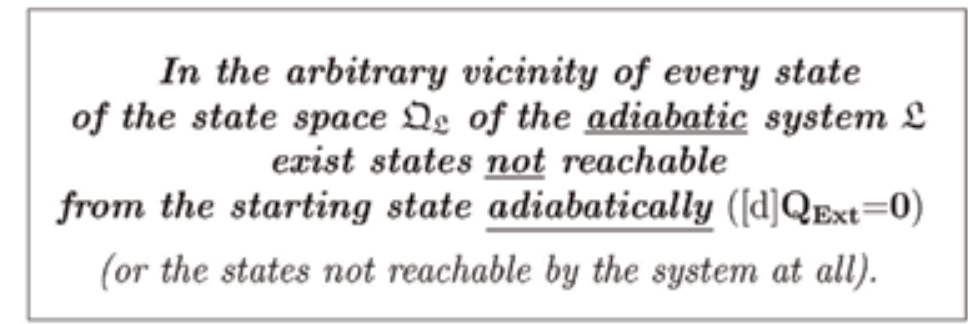

For the consistency of the Peano arithmetic theory $\mathcal{T}_{\mathcal{P} \mathcal{A}}$ the analog is expressed by:

Gödel incompletness theorems:

For the theory $\mathcal{T}_{\mathcal{P A}}$ exists the true ("1") CLAIM that either this CLAIM and its NEGATION is NOT PROVABLE within the system $\mathcal{P} / \mathcal{T}_{\mathcal{P A}}$.

- CLAIM about the $\mathcal{T}_{\mathcal{P} A}$ consistency especially -

$$
\begin{aligned}
& \text { The CLAIM saying that theory } \mathcal{T}_{\mathcal{P} A} \text { is consistent } \\
& \text { is not PROVABLE by its means }(\mathcal{P}) \text { - by itself. }
\end{aligned}
$$

In our considerations, we use the states of the adiabatic system as the thermodynamic representation of the Peano arithmetically inferred formulas and the transition between the stats is then the thermodynamic model of the Peano arithmetic inference step, the consistency of the Peano arithmetics is represented by the adiabacity of the modeling thermodynamic system. 
Peano Axioms/Inference Rules in the System $\mathcal{P} /$ Theory $\mathcal{T}_{\mathcal{P}_{\mathcal{A}}}$.

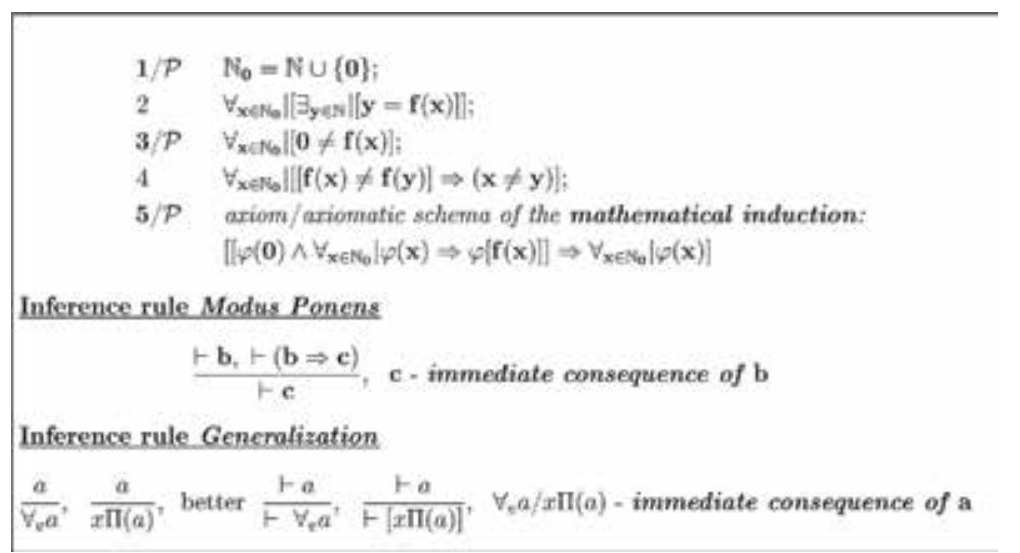

4 " 1 " - arithmeticity of the $\mathcal{P} \cong$ adiabacity of the $\mathfrak{L} / \mathfrak{O}_{\mathfrak{L}}$.

\& Consistent $\mathcal{T}_{\mathcal{P A}}$ inference within $\mathcal{P} \cong$ moving along trajectories $1_{\mathfrak{D} \mathfrak{L}}$ in $\mathfrak{D}_{\mathfrak{L}} / \mathfrak{L}$.

\& The states on the adiabatic trajectories, also irreversible, then model the consistently inferred/inferrable PA-FORMULAS.

Remark: Any inference within the system $\mathcal{P}^{2}$ sets the $\mathcal{T}_{\mathcal{P A}_{\mathcal{A}} \text {-theoretical relation }}{ }^{3}$ among its formulae $a_{[\cdot]}$. This relation is given by their gradually generated special sequence $\vec{a}\left[a_{1}, \ldots, a_{q}, \ldots, a_{p}, \ldots, a_{k}, a_{k+1}\right]$, which is the proof of the latest inferred formula $a_{k+1}$. By this, the unique arithmetic relation between their Gödel numbers, FORMULAE $x_{[\cdot]}, x_{[\cdot]}=\Phi\left(a_{[\cdot]}\right)$, is set up, too. The gradually arising SEQUENCE of FORMULAE $x=\Phi(\vec{a})$ is the PROOF of its latest FORMULA $x_{k+1}$.

Let us assume that the given sequence $\vec{a}=\left[a_{01}, a_{02}, \ldots, a_{o}, \ldots, a_{q}, \ldots, a_{p}, \ldots, a_{k}, a_{k+1}\right]$ is a special one, and that, except of axioms (axiomatic schemes) $a_{01}, \ldots, a_{o}$, it has been generated by the correct application of the rule Modus Ponens only. ${ }^{4}$

Within the process of the (Gödelian) arithmetic-syntactic analysis of the latest formula $a_{k+1}$ of the proof $\vec{a}$, we use, from the $\vec{a}$ selected, (special) subsequence $\overrightarrow{a_{q, p, k+1}}$ of the formulae $a_{q}, a_{p}, a_{k+1}$. The formulae $a_{q}, a_{p}$ have already been derived, or they are axioms. It is valid that $q, p<k+1$, and we assume that $q<p$,

$$
\begin{aligned}
\overrightarrow{a_{q, p, k+1}} & =\left[a_{q}, a_{p}, a_{k+1}\right], \quad a_{p} \cong a_{q} \supset a_{k+1}, \overrightarrow{a_{q, p, k+1}}=\left[a_{q}, a_{q} \supset a_{k+1}, a_{k+1}\right], \\
x & =\Phi(\vec{a})=\Phi\left(\left[\Phi\left(a_{1}\right), \Phi\left(a_{2}\right), \ldots, \Phi\left(a_{q}\right), \ldots, \Phi\left(a_{p}\right), \ldots, \Phi\left(a_{k}\right), \Phi\left(a_{k+1}\right)\right]\right) \\
& =\Phi(\vec{x})=\Phi\left(x_{1}\right) * \Phi\left(x_{2}\right) * \ldots * \Phi\left(x_{q}\right) * \ldots * \Phi\left(x_{q}\right) * \ldots * \Phi\left(x_{k}\right) * \Phi\left(x_{k+1}\right) \\
l(x) & =l[\Phi(\vec{x})]=l[\Phi(\vec{a})]=k+1, \\
x_{k+1} & =\Phi\left(a_{k+1}\right)=l[\Phi(\vec{a})] G l \Phi(\vec{a})=(k+1) G l x \\
x_{p} & =\Phi\left(a_{p}\right)=\Phi\left(a_{p} \supset a_{k+1}\right)=q G l \Phi(\vec{a}) * \Phi(\supset) * l[\Phi(\vec{a})] G l \Phi(\vec{a}) \\
& =q G l x \operatorname{Imp}[l(x)] G l x \\
x_{q} & =\Phi\left(a_{q}\right)=q G l \Phi(\vec{a})=q G l x
\end{aligned}
$$

\footnotetext{
${ }^{2}$ Formal arithmetic inferential system.

3 Peano Arithmetics Theory.

${ }^{4}$ For simplicity. The 'real' inference is applied to the formula $a_{i+1}$ for $i=o$.
} 


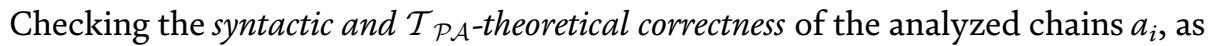
the formulae of the system $\mathcal{P}$ having been generated by inferring (Modus Ponens) within the system $\mathcal{P}$ (in the theory $\mathcal{T}_{\mathcal{P A}}$ ), and also the special sequence of the formulae $\vec{a}$ of the system (theory $\mathcal{T}_{\mathcal{P A}_{\mathcal{A}}}$ ), is realized by checking the arithmeticsyntactic correctness of the notation of their corresponding FORMULAE and SEQUENCE of FORMULAE, by means of the relations Form $(\cdot), F R(\cdot), O p(\cdot, \cdot, \cdot), F l$ $(\cdot, \cdot, \cdot)$ "called" from (the sequence of procedures) relations Bew $(\cdot),(\cdot \cdot) B(\cdot), B w(\cdot) ;^{5}$ the core of the whole (Gödelian) arithmetic-syntactic analysis is the (procedure) relation of Divisibility,

$$
\begin{aligned}
& \operatorname{Form}\left[\Phi\left(a_{i}\right)\right]=" 1 " / 0 ", \quad F R\left[\Phi\left(\overrightarrow{a_{1}^{i+1}}\right)\right]=" 1 " / " 0 ", \quad o \leq i \leq k \\
& O p\left[x_{k}, \operatorname{Neg}\left(x_{q}\right), x_{k+1}\right]=O p\left[\Phi\left(a_{p}\right), \Phi\left[\sim\left(a_{q}\right)\right], \Phi\left(a_{k+1}\right)\right]=" 1 " / 0 " \\
& F l[(k+1) G l x, p G l x, q G l x]=" 1 " / " 0 " \\
& x B x_{k+1}=" 1 " / " 0 ", \operatorname{Bew}\left(x_{k+1}\right)=\text { "1"/0"; } \\
& \Phi\left(a_{p}\right)\left\|23^{3 G l \Phi\left(\left(\overrightarrow{a_{q, p, k+1}}\right)\right.} \& \boldsymbol{\Phi}\left(a_{p}\right)\right\| 7^{1 G l \Phi(}\left(\overrightarrow{a_{q, p, k+1}}\right)=" 1 " / " 0 "
\end{aligned}
$$

\section{Gödel theorems}

Remark: The expression $S b\left(\begin{array}{ccc} & u_{1} & u_{2} \\ t & & \\ Z(x) & Z(y)\end{array}\right)$ or the expression

$S b\left(\begin{array}{ccc} & 17 & 19 \\ t & & \\ & Z(x) & Z(y)\end{array}\right)$ represents the result value of the Gödel number $t[Z(x), Z(y)]$,

which is coding the (constant) claim $T(x, y)$ z $P M$ has been generated by the substitution of $x$ a $y$ instead of the free variables $X$ and $Y$ in the function $T(X, Y)$ from $P M$ with its Gödelian code $t\left(u_{1}, u_{2}\right)$ in the (arithmetized) $\mathcal{P}$,

$$
S b\left(\begin{array}{ccc} 
& u_{1} & u_{2} \\
t & & \\
& Z(x) & Z(y)
\end{array}\right)=S b\left(\begin{array}{ccc} 
& 17 & 19 \\
t & & \\
& Z(x) & Z(y)
\end{array}\right)
$$

\& Into the VARIABLES, we substitute the SIGNS of the same type but the introduction of the term admissible substitution itself is not supposing it wordly.

- Then it is possible to work even with the expressions not grammatically correct and thus with such chains, which are not FORMULAE of the system $\mathcal{P}$ (and thus not belonging into the theory $\mathcal{T}_{\mathcal{P A}_{\mathcal{A}}}$ ).

Then the substitution function $S b\left(\begin{array}{l}. \\ . \\ \ldots\end{array}\right)$ is not possible, within the frame of the inference in the system $\mathcal{P}$, be used isolately as an arbitrarily performed number manipulation-in spite of the fact that it is such number manipulation really. It is used only and just within the frame of the language $\mathcal{L}_{\mathcal{P}}$ and, above all, within the frame of the conditions specified by the právě a jenom

\footnotetext{
${ }^{5}$ Formula, Reihe von Formeln, Operation, Folge, Glied, Beweis, Beweis, see Definition 1-46 in [5-7] and by means of all other, by them 'called', relations and functions (by their procedures).
} 
INFERENCE of the elements of the language $\mathcal{L}_{\mathcal{T}_{\mathcal{P A}}}$ only (and thus in the more limited way).

Others than/semantically (or by the type) homogenous application of the substitution function is not within the right inference/INFERENCE within the system $\mathcal{P}$ possible. ${ }^{6}$

\subsection{The Gödel UNDECIDABLE CLAIM's construction}

Let the Gödel numbers $x$ and $y$ be given. The number $x$ is the SEQUENCE OF FORMULAE valid and $y$ is a FORMULA of $\mathcal{P}$. We define the valid constant relation $Q(x, y)$ from the $Q(X, Y)$ for given values $x$ and $y, X:=x, Y:=y ; 17=\Phi(X)$, $19=\Phi(Y){ }^{7,8}$

$$
\begin{aligned}
& Q(x, y) \equiv x B_{\kappa}\left[S b\left(\begin{array}{c}
19 \\
y \\
Z(p)
\end{array}\right)\right] \equiv B e w_{\kappa}\left[S b\left(\begin{array}{ccc} 
& 17 & 19 \\
q & & \\
& Z(x) & Z(y)
\end{array}\right)\right] \\
& q[Z(x), Z(y)]=\Phi[Q(x, y)], \overline{x B_{\kappa} y^{\prime}} \equiv \operatorname{Bew}_{\kappa}\left(y^{\prime}\right)=\operatorname{Bew}_{\kappa}[y[Z(y)]]=\operatorname{Bew}_{\kappa}[q[Z(x), Z(y)]]
\end{aligned}
$$

Now we put $p=17$ Gen $q, q=q(17,19)[q(17,19) \triangleq Q(X, Y)]$ and then,

$$
p=17 \operatorname{Gen} q(17,19)=\Phi\left[\forall_{x \in \mathbb{X}} \mid Q(x, Y)\right] \triangleq Q(\mathbb{X}, Y) \triangleq Q\left(\mathbb{N}_{0}, Y\right)
$$

The meta-language symbol $Q(\mathbb{X}, Y)$ or $Q\left(\mathbb{N}_{0}, Y\right)$ is to be read: No $x \in \mathbb{X}\left(\mathbb{N}_{0}\right)$ is in the $\kappa$-INFERENCE relation to the variable $Y$ (to its space of values $\mathbb{Y}$ ).

$\checkmark$ Further, with the Gödel substitution function, we put $q[17, Z(p)]=r(17)=r$,

$$
r:=S b\left(\begin{array}{c}
19 \\
q \\
Z(p)
\end{array}\right) \text { and then } r=S b\left(\begin{array}{cc}
q(17,19) & \\
& Z(p)
\end{array}\right)=r(17)=\Phi[Q(X, p)]
$$

The Gödel number $r$ is, by the substitution of the NUMERAL $Z(p)$, supposedly only (by [5-7]) the CLASS SIGN with the FREE VARIABLE $17(X)$; with the values $p$, the $r$ contains the feature of autoreference,

$$
\begin{aligned}
r & =r(17)=q[17, Z[p(19)]]=q[17, Z[17 \operatorname{Gen} q(17,19)]] \triangleq Q(X, p) \\
& =\Phi\left[Q\left[X, \quad \Phi\left[\forall_{x \in X} \mid Q(x, Y)\right]\right]\right]_{Y:=p} \triangleq Q[X, \quad \Phi[Q(\mathbb{X}, Y)]] \triangleq Q\left[X, \quad \Phi\left[Q\left(\mathbb{N}_{0}, Y\right)\right]\right]
\end{aligned}
$$

- Within the Gödel number/code $q, q=q[17,19]$, we perform the substitution $Y:=p$ and then $X:=x$ and write

${ }^{6}$ Substitution function $S b\left(\begin{array}{l}. \\ \cdots \\ \ldots\end{array}\right)$ is, in this way, similar to the computer machine instruction which itself, is always able to realize its operation with its operands on the arbitrary storage place, but practically it is always applicated within the limited address space and within the given operation regime/mode of the computer's activity only (e.g. regime/mode Supervisor or User).

$7 \Phi$ and $Z$ represents the Gödel numbering and $S b$ the Substitution, B, Bew the PA-arithmetic Proof.

${ }^{8}$ Following the Gödel Proposition $V$ (the first part) [5-7]. 


$$
\begin{aligned}
r[Z(x)] & =S b\left(\begin{array}{rrr} 
& 17 & 19 \\
q(17,19) & & \\
& Z(x) & Z(p)
\end{array}\right)=S b\left(\begin{array}{ll}
17, Z(p)] & 17 \\
Z(x)
\end{array}\right) \\
& =\Phi[Q[x, \Phi[Q(\mathbb{X}, \quad Y)]]]=\Phi\left[Q\left[x, \quad \Phi\left[Q\left(\mathbb{N}_{0}, \quad Y\right)\right]\right]\right]=\Phi[Q(x, p)]
\end{aligned}
$$

With the great quantification of $r[Z(x)]$ by $Z(x)$ by the VARIABLE $X$ (17), we have (similarly as in $[4,8]$ ),

$$
\begin{gathered}
Z(x) \operatorname{Genr}[Z(x)]=17 \operatorname{Gen} q[17, Z[17 \operatorname{Genq}(17,19)]]=17 \operatorname{Gen} r(17)=17 \operatorname{Genr} \\
\triangleq \Phi\left[\forall_{x \in X} \mid \Phi\left[Q\left[x, \Phi\left[\forall_{x \in X} \mid Q(x, Y)\right]\right]\right]\right]=\Delta Q[\mathrm{X}, \Phi[Q(\mathrm{X}, Y)]]=Q\left[\mathbb{N}_{0}, \Phi\left[Q\left(\mathbb{N}_{0}, Y\right)\right]\right]
\end{gathered}
$$

\subsection{Gödel theorems}

I. Gödel theorem (corrected semantically by $[3,9,10]$ ) claims that

\& for every recursive and consistent CLASS OF FORMULAE $\kappa$ and outside this set there is such true (" 1 ") CLAIM $r$ with free VARIABLE $v[r \triangleq r(v)]$ that neither PROPOSITION $v$ Gen $r$ nor PROPOSITION Neg $(v$ Gen $r)$ belongs to the set $F \lg (\kappa)$,

$$
[v \operatorname{Gen} r \notin F l g(\kappa)] \&[\operatorname{Neg}(v \operatorname{Gen} r) \notin F \lg (\kappa)]
$$

FORMULA $v G e n r$ and $N e g(v G e n r)$ are not $\kappa$-PROVABLE-FORMULA $v$ Gen $r$ is not $\kappa-D E C I D A B L E$. They both are elements of inconsistent (meta)system $\mathcal{P}^{*}$.

II. Gödel theorem (corrected semantically according to $[3,9,10]$ ) claims that

\& if $\kappa$ is an arbitrary recursive and consistent CLASS OF FORMULAE, then any CLAIM saying that CLASS $\kappa$ is consistent must be constructed outside this set, and for this fact it is not $\kappa-P R O V A B L E$.

- Outside ${ }^{9}$ the consistent system $\mathcal{P}_{\kappa}$, there is a true (" 1 ") formula, ${ }^{10}$ the ARITHMETIZATION of which is $\kappa$-UNPROVABLE FORMULA 17Gen $r^{11}$

$\checkmark$ The fact that the recursive CLASS OF FORMULAE $\kappa$ (now PA-Peano Arithmetic especially) is consistent, is tested by unary relation Wid $(\kappa)$, (die Widerspruchsfreiheit, Consistency) [5-7],

$$
\operatorname{Wid}(\kappa) \sim(E x)\left[\operatorname{Form}(x) \& \overline{\operatorname{Bew}_{\kappa}(x)}\right]
$$

- a class of FORMULAE $\kappa$ is consistent $\stackrel{\text { Def }}{\Leftrightarrow}$ there exists at least one FORMULA $x$ [PROPOSITION $x(x=17$ Gen $r)]$, which is $\kappa$-UNPROVABLE.

\section{Caratheodory theorems}

I. Caratheodory's theorem $(\Rightarrow)$ says that: $\diamond$ If the Pfaff form has an integration factor, then there are, in the arbitrary vicinity of any arbitrarily chosen and fixed point

\footnotetext{
9 Far from (!) “In ...." in [5-7]

10 Far from “... [PA-] arithmetic and sentencial/SENTENCIAL” in [5-7].

11 Any attempt to prove/TO PROVE it (to infer/to TO INFER it) in the system $\mathcal{P}_{\kappa}$ assumes or leads to the requirement for inconsistency of the consistent (!) system $\mathcal{P}_{\kappa}$ (in fact we are entering into the inconsistent metasystem $\mathcal{P}^{*}$ - see the real sense [4, 9] of the Proposition $V$ in [5-7]).
} 
P of the hyperplane $\mathcal{R}\left[P \in \mathcal{R}\left[\left(x_{i}\right)_{i=1}^{n}\right]=\right.$ const. $]$, such points which, from this point $P$, are inaccessible along the path satisfying the equation $\mathrm{d} Q=0$.

II. Caratheodory theorem $(\Leftarrow)$ says that: $\diamond$ If the Pfaff form $\delta Q=\sum_{i=1}^{n} X_{i} \mathrm{~d} x_{i}$, where $X_{i}$ are continuously differentiable functions of $n$ variables (over a simply continuous area), has such a property that in the arbitrary vicinity of any arbitrarily chosen and fixed point $P$ of the hyperplane $\mathcal{R}\left[P \in \mathcal{R}\left[\left(x_{i}\right)_{i=1}^{n}\right]=\right.$ const. $]$, there exists such points which, from $P$, cannot be accessible along the path satisfying the equation $\mathrm{d} Q=0$, then this form is holonomous; it has or it is possible to find an integration factor for it.

Caratheodory formulation of the II. Law of Thermodynamics $(\Leftrightarrow)$ claims that:

$\checkmark$ In the arbitrary vicinity of every state of the state space of the adiabatic system, there are such states that, from the given starting point, cannot be reached along an adiabatic path (reversibly and irreversibly), or such states which the system cannot reach at all, see the Figure 1.

Remark: Now the symbol $Q$ denotes that heat given to the state space of the thermodynamic system from its outside and directly; $Q \triangleq Q_{E x t}$; along paths $l_{2 b}, l_{2 b}$, $l_{2 d}, l_{2 e}, l_{3}$ is $Q_{E x t}=0, \Delta Q_{E x t}=0, \mathrm{~d} Q_{E x t}=0$.

\& The states' $\theta_{[\cdot]}^{\mathfrak{L}}$ changes in the adiabatic system $\mathfrak{L} / \mathfrak{O}_{\mathfrak{L}}$, along the trajectories $1_{\mathfrak{O}_{\mathfrak{L}}}$ are expressible regularly:

$l_{2 b}$ isothermic irreversible, $\quad \boldsymbol{\theta}_{1}^{\mathfrak{L}} \rightarrow \Delta A_{1,2 e} \boldsymbol{\theta}_{2 e}^{\mathfrak{L}}$

$l_{2 b^{\prime}}$ adiabatic irreversible, $\quad \boldsymbol{\theta}_{1}^{\mathfrak{L}} \rightarrow \Delta A_{1,3} \boldsymbol{\theta}_{3}^{\mathfrak{L}}$

$l_{2 d}$ izobaric irreversible, $\quad \boldsymbol{\theta}_{1}^{\mathfrak{L}} \rightarrow \Delta A_{1,2 b} \boldsymbol{\theta}_{2 b}^{\mathfrak{Q}}$

$l_{2 c}$ izentropic reversible, $\quad \boldsymbol{\theta}_{1}^{\mathcal{L}} \rightarrow \Delta A_{1,2 b^{\prime}} \boldsymbol{\theta}_{2 b^{\prime}}^{\mathcal{L}}$

$l_{3}$ izochoric irreversible, $\quad \boldsymbol{\theta}_{1}^{\mathfrak{L}} \rightarrow \Delta A_{1,2 d} \boldsymbol{\theta}_{2 d}^{\mathfrak{L}}$

$\boldsymbol{\theta}_{1}^{\mathfrak{L}} \rightarrow \lambda \boldsymbol{\theta}_{1}^{\mathfrak{L}}$

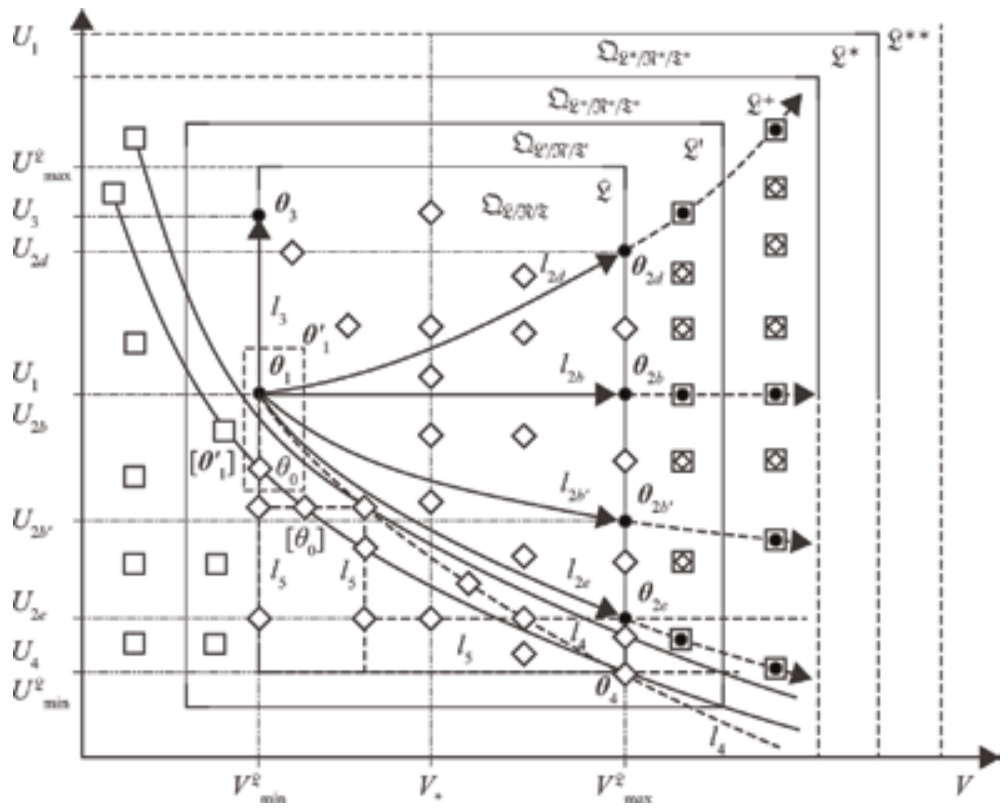

Figure 1.

Adiabatic changes of the state of the system $\mathfrak{L}$, illustration. 
Through the state space of FORMULAE of the system $\mathcal{P}$, we "travel" similarly by the inference rules, Modus Ponens especially [performed by a Turing Machine TM, the inference of which is considerable as realized by the information transfer process within a Shannon Transfer Chain $(\mathbf{X}, \mathcal{K}, \mathbf{Y})$ described thermodynamically by a Carnot Machine $C M]$.

The thermodynamic model for the consistent $\mathcal{P} / \mathcal{T}_{\mathcal{P A}}$ inference, from its axioms or formulas having been inferred so far, is created by the Carnot Machine's activity, which models the inference. This whole Carnot Machine $C M$ runs in the wider adiabatic system $\mathfrak{L} / \mathfrak{D}_{\mathfrak{L}}$ and, in fact, is, in this way, creating these states, [the $T M$ 's, $(\mathbf{X}, \mathcal{K}, \mathbf{Y})^{\text {'s }}$, configurations are then modeled by the states $\boldsymbol{\theta}_{i}^{\mathfrak{L}} \in \mathfrak{O}_{\mathfrak{L}}$ of the adiabatic $\mathfrak{L} / \mathfrak{D}_{\mathfrak{L}}$ with this modeling $\boldsymbol{C M}$ inside], see the Figure 2.

The $\mathfrak{L}^{\prime}$ 's initial imbalance starts the $\theta_{[\cdot]}^{\mathfrak{L}}$ s states' sequence on a trajectory $l_{\mathfrak{O}}$ and is given by the modeled

$$
\begin{aligned}
& \text { temperature difference } \mathrm{T}_{\mathrm{w}}-\mathrm{T}_{0}>0 \text { on } C M, \\
& \text { existence of the input message on } \mathcal{K}, \\
& \text { input chain's existence on the } T M \text { 's input-output tape }
\end{aligned}
$$

These adiabatic trajectories $1_{\mathfrak{O}_{\mathfrak{L}}}$ now represent the norm of the consistency

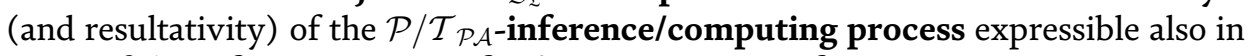
terms of the information transfer/heat energy transformation.

\& The adiabatic property of the thermodynamic system $\mathfrak{L}$ is always created over the given scales of its state quantities-over their scale for a certain "creating" original (and not adiabatic) system $\mathfrak{T}$, and by its outerly specification or the design/ construction by means of heat/adiabatic isolation of the space $V_{\max }$ of the original system $\mathfrak{T}$ that the system $(\mathfrak{L} / \mathfrak{T})$ can occupy, and after the system $\mathfrak{L}$ has been (as the adiabatic isolated original system $\mathfrak{T}$ ) designed and set in the starting state $\theta_{1}$, see Figure 1 . The state $\theta_{4}$ is a state $\diamond$ of the set of states $\{\diamond\}$. These states are those ones in the Figure 1, which, although they are in the given scale of state quantities $U$ and $V$ of the state space $\mathfrak{O}_{\mathfrak{L}}$ of the system $\mathfrak{L}$ considered, $U \in\left\langle U_{\min }, U_{\max }\right\rangle$ and $V \in\left\langle V_{\min }, V_{\max }\right\rangle$, are within it [in (the state space $\mathfrak{O}_{\mathfrak{L}}$ of) $\mathfrak{L}$ ]

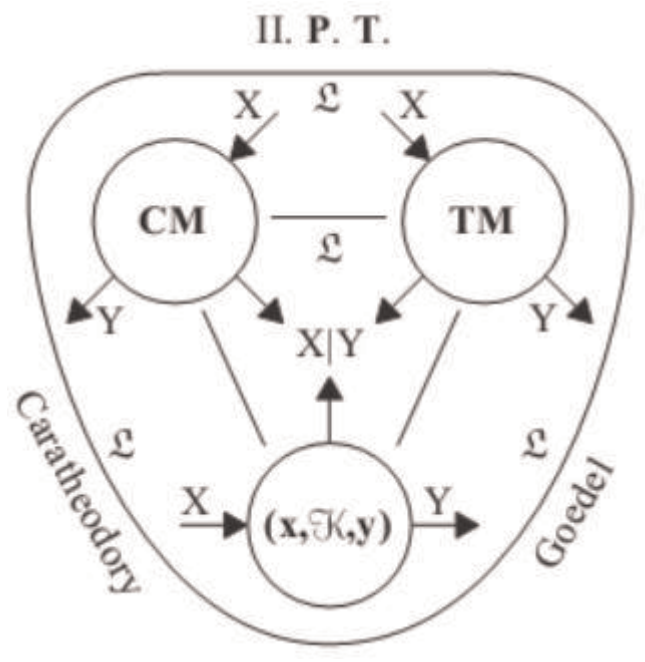

Figure 2.

The mutual describability of the $C M,(X, \mathcal{K}, Y)$ and $T M$. 
by permitted (adiabatic, $\mathrm{d} Q_{E x t}=0$ ) changes $l_{2 b}, l_{2 b^{\prime}}, l_{2 d}, l_{2 e}$ and $l_{3}$, inaccessible. And certainly, thermodynamic states $\square$ beyond these scales, within the hierarchically higher systems, are not accessible from the inside of the system $\mathfrak{L} / \mathfrak{T}$ itself, without its (not adiabatical) widening, either, see the Figure 1.

. Without violation of the adiabacity of the system $\mathfrak{L}$, it is not possible to reach the state $\boldsymbol{\theta}_{4}$ from the state $\boldsymbol{\theta}_{1}$ along any simple path $l_{2 b}, l_{2 b^{\prime}}, l_{2 d}, l_{2 e}$ in the state space $\mathfrak{O}_{\mathfrak{L}}$,

\& However, outside the adiabacity of the system $\mathfrak{L}$ expressed by the relation $\mathrm{d} Q_{E x t}=0$, which means under the opposite requirement $\mathrm{d} Q_{E x t} \neq 0$, it is possible to design or to construct a (nonadiabatic) path linking a certain point/state of the state space $\mathfrak{O}_{\mathfrak{L}}$ located, e.g., on $l_{2 e}$ with the point/state $\theta_{4}$; for example, it is the path $l_{4}$ from $\boldsymbol{\theta}_{\mathbf{1}}$ to $\boldsymbol{\theta}_{\mathbf{4}}$, now in a certain nonadiabatic system $\mathfrak{N}$, $\mathfrak{N} \subseteq \mathfrak{T}$ where, from the view of possibilities of changes of the state, see Figure 1, is valid that

$$
\begin{aligned}
& \square \notin \mathfrak{O}_{\mathfrak{L} / \mathfrak{T}},\{\square\} \nsubseteq \mathfrak{O}_{\mathfrak{L} / \mathfrak{T}} ; \quad \diamond \notin\left\{l_{2 b}, l_{2 b^{\prime}}, l_{2 d}, l_{2 e}, l_{3}\right\}, \quad\{\diamond\} \varsubsetneqq \mathfrak{O}_{\mathfrak{L} / \mathfrak{T}} \\
& \mathfrak{O}_{\mathfrak{L}}=\mathfrak{O}_{\mathfrak{N}}=\mathfrak{O}_{\mathfrak{T}} \triangleq \mathfrak{O}_{\mathfrak{L} / \mathfrak{T}} \mathfrak{T} \supseteqq \mathfrak{N} \supsetneqq \mathfrak{L}
\end{aligned}
$$

. Further, it is possible to create for this nonadiabatic system $\mathfrak{N}$ an alternative adiabatic system $\mathfrak{L}^{\prime}\left(\mathfrak{D}_{\mathfrak{L}^{\prime}} \supseteqq \mathfrak{D}_{\mathfrak{L}}\right)$ enabling adiabatic-isochoric changes, e.g., $\boldsymbol{\theta}_{2 e} \rightarrow \boldsymbol{\theta}_{4}$.

.. Both the new adiabatic system $\mathfrak{L}^{\prime}$ and its nonadiabatic "model” $\mathfrak{N}$ can be a subsystem of another but also adiabatic and imminently superior system $\mathfrak{L}^{+}$having another/wider range of the state quantities than it was for the original systems $\mathfrak{L}$ and $\mathfrak{N},\left(\mathfrak{O}_{\mathfrak{L}} \subseteq \mathfrak{O}_{\mathfrak{L}^{\prime} / \mathfrak{N}} \subseteq \mathfrak{O}_{\mathfrak{L}^{+}}\right)$. Then the path $l_{4}$ in the state space $\mathfrak{O}_{\mathfrak{L}^{\prime} / \mathfrak{N}}$ of the system $\mathfrak{L}^{\prime} / \mathfrak{N}$ will be, from the point of $\mathfrak{L}^{\prime}$ of the imminently superior adiabatic system $\mathfrak{L}^{+}$, the adiabatic one-the system $\mathfrak{L}^{\prime}$ is already isolated in $\mathfrak{L}^{+}$and the system $\mathfrak{L}^{+}$itself is already created in a certain system $\mathfrak{L}^{*}$ imminently superior to it, as an isolated/ adiabatic substitute for the system $\mathfrak{N}^{\prime}\left(\mathfrak{O}_{\mathfrak{L}^{\prime} / \mathfrak{N}} \varsubsetneqq \mathfrak{O}_{\mathfrak{L}^{+}}, \mathfrak{O}_{\mathfrak{L}^{+} / \mathfrak{N}^{\prime}} \varsubsetneqq \mathfrak{O}_{\mathfrak{L} *} \ldots\right)$.

.. From the view of the possibilities to change the state, or from the view of the energetic relations $(\mathscr{E})$, it is possible, see the Figure 1, to write,

$$
\mathfrak{L} \varsubsetneqq \mathfrak{L}^{\prime} \varsubsetneqq \mathfrak{L}^{+} \varsubsetneqq \mathfrak{L}^{*} \varsubsetneqq \mathfrak{L}^{* *} \ldots, \quad \mathfrak{N} \varsubsetneqq \mathfrak{N}^{\prime} \varsubsetneqq \mathfrak{N}^{+} \varsubsetneqq \mathfrak{N}^{*} \varsubsetneqq \mathfrak{N}^{* *} \ldots
$$

$\left\{\mathfrak{N}^{\prime} \varsubsetneqq \mathcal{L}^{+}\right\}_{\mathcal{E}}, \mathfrak{N}$ is implemented in $\mathfrak{L}^{\prime},\left\{\mathfrak{N}^{+} \varsubsetneqq \mathfrak{L}^{*}\right\}_{\mathcal{E}}, \mathfrak{N}^{\prime}$ is implemented in $\mathfrak{L}^{+}$ $\left\{\mathfrak{N}^{+} \varsubsetneqq \mathfrak{L}^{*}\right\}_{\mathcal{E}}, \mathfrak{N}^{+}$is implemented in $\mathrm{v} \mathfrak{L}^{*}, \ldots$

We introduce a symbol $l_{\mathfrak{O}_{\mathfrak{L}}[\cdot]}$ for adiabatic paths in the state spaces $\mathfrak{O}_{\mathfrak{L}[\cdot]}$,

$l_{\mathfrak{Q}_{\mathfrak{E}}} \triangleq\left\{l_{2 b}, l_{2 b^{\prime}}, l_{2 d}, l_{2 e}, l_{3}\right\}, \quad l_{\mathfrak{Q}_{\mathfrak{I}^{\prime}}} \triangleq\left\{l_{2 b}, l_{2 b^{\prime}}, l_{2 d}, l_{2 e}, l_{3}, l_{2 e}-l_{\theta_{2 e}, \theta_{4}}, l_{5}\right\}, \quad l_{\mathfrak{Q}_{\mathfrak{E}}+}, l_{\mathfrak{Q}_{\mathfrak{R}^{*}}}, \ldots$

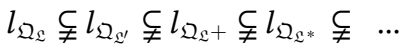

\& The states from the sets $\left\{\mathfrak{O}_{\mathfrak{L}}-l_{\mathfrak{Q}_{L}}\right\},\left\{\mathfrak{O}_{\mathfrak{L}^{\prime}}-l_{\mathfrak{Q}_{\mathfrak{L}}}\right\},\left\{\mathfrak{Q}_{\mathfrak{L}+}-l_{\mathfrak{Q}_{\mathfrak{L}}}\right\}$, $\left\{\mathfrak{O}_{\mathfrak{L}^{*}}-l_{\mathfrak{Q}_{\mathfrak{L}}}\right\}, \ldots$ in the view of adiabacity and specification of the system $\mathfrak{L}$ are forming, within the hierarchy of the systems $\mathfrak{L}, \mathfrak{L}^{\prime}, \mathfrak{L}^{+}, \mathfrak{L}^{*}, \ldots$, a certain set $\mathfrak{O}_{\mathfrak{L}}^{*}=\{\{\diamond\} \cup\{\square\}\}$, which is in the framework of the system $\mathfrak{L}$ inaccessible/ unachievable as a whole and also in any of its subset and member. However, the $\mathfrak{L}$-inaccessibility (adiabatic inaccessibility, especially of $\{\diamond\}$ in the state space $\mathfrak{O}_{\mathfrak{L} / \mathfrak{T}}$ ) also means existence of the paths $l_{\mathfrak{Q}}$ of the adiabatic system $\mathfrak{L}$. In the sense of the domain of solution of its (the $\mathfrak{L}$ 's) state equations, they cannot be part of the functionality of $\mathfrak{L}$ (but mark it). 


\section{Analogy between adiabacity and $P A$-inference}

\& Now the states on the adiabatic paths $l_{\mathfrak{Q}_{\mathfrak{E}}}$ (of changes of the state of the adiabatic system $\mathfrak{L}$ ) are considered to be the analogues of $P A$-arithmetic claims/ claims of the Peano Arithmetic theory $\mathcal{T}_{\mathcal{P A}}$ (formulated/inferred/proved in $\mathcal{P}$ ),

- adiabacity of the system $\mathfrak{L}$ is the analog of consistency of the system $\mathcal{P}_{[\kappa]}$ and

. the set $l_{\mathfrak{Q}_{\mathfrak{L}}}$ of adiabatic paths in $\mathfrak{O}_{\mathfrak{L} / \mathfrak{T}}$ is an analog of $P A$-theory $\mathcal{T}_{\mathcal{P} \mathcal{A}}$; then, adiabatic analogy of the higher consistent inferential system $\mathcal{P}^{\prime}$ is by $\mathfrak{L}^{\prime}, \mathcal{P}^{\prime} \supsetneqq \mathcal{P}, \ldots$.

.. Then the given specific adiabatic path $l_{2 b}, l_{2 b^{\prime}}, l_{2 d}, l_{2 e}, l_{3}$ is an analog of certain deducible thread $\vec{x} B x_{k}$ of the claim $x_{k}$ of the theory $\mathcal{T}_{\mathcal{P A}_{\mathcal{A}}}$, where

$$
\begin{gathered}
\vec{x} B x_{k}=\left(x_{1}, x_{2}, \ldots, x_{k-1}, x_{k}\right) B x_{k}=" 1 " \\
x_{1} \in\{A X I O M S\}^{\mathcal{P}} \text { and } x_{1} \cong \boldsymbol{\theta}_{1} \\
x_{1}, x_{2}, \ldots, x_{k-1}, x_{k} \in \mathcal{T}_{\mathcal{P} \mathcal{A}} \text { and } x_{k} \cong \boldsymbol{\theta} \in\left\{\boldsymbol{\theta}_{2 b}, \boldsymbol{\theta}_{2 b^{\prime}}, \boldsymbol{\theta}_{2 d}, \boldsymbol{\theta}_{2 e}, \boldsymbol{\theta}_{3}\right\} \\
x_{2}, \ldots, x_{k-1} \cong \boldsymbol{\theta} \in\left\{\left\{\left\{l_{2 b}-\boldsymbol{\theta}_{2 b}\right\},\left\{l_{2 b^{\prime}}-\boldsymbol{\theta}_{2 b^{\prime}}\right\}, \quad\left\{l_{2 d}-\boldsymbol{\theta}_{2 d}\right\},\right.\right. \\
\left.\left.\left\{l_{2 e}-\boldsymbol{\theta}_{2 e}\right\},\left\{l_{3}-\boldsymbol{\theta}_{3}\right\}\right\}-\boldsymbol{\theta}_{1}\right\}
\end{gathered}
$$

\& The states from the space $\mathfrak{Q}_{\mathfrak{L} / \mathfrak{T}}$ of the system $\mathfrak{L} / \mathfrak{T}$ satisfying the range of values of the state quantities $p \in\left\langle p_{\min }, p_{\max }\right\rangle, V \in\left\langle V_{\min }, V_{\max }\right\rangle, T \in\left\langle T_{\min }, T_{\max }\right\rangle /$ $\left.U \in\left\langle U_{\min }, U_{\max }\right\rangle\right)$, which are inaccessible along any of the adiabatic paths from $l_{\mathfrak{Q}_{\mathrm{L}}}$, that means they are the states $\diamond$ from the difference $\left\{\mathfrak{O}_{\mathfrak{L} / \mathfrak{T}}-l_{\mathfrak{Q}_{\mathfrak{L}}}\right\}$, shortly said from $\{\mathfrak{T}-\mathfrak{L}\}$, are considered to be analogues of not $P A$-claims such as, e.g., the Fermat's Last Theorem. ${ }^{12}$ So, they are analogues of all-the-time true (" 1 ") arithmetic but not- $P A$-arithmetic claims. From the point of adiabacity of the system $\mathfrak{L}$, they $(\diamond)$ are only some thermodynamic states of its "creating" system $\mathfrak{T}$, and they are from the common range of values of the state quantities for $\mathfrak{T}$ and $\mathfrak{L}$. From the point of expressing possibilities it as always true

$$
\mathfrak{L}^{[\cdot]} \varsubsetneqq \mathfrak{N}^{[\cdot]} \subseteq \mathfrak{T}^{[\cdot]} \varsubsetneqq \mathfrak{T}^{\star} \varsubsetneqq\left\{\mathfrak{Q}_{\mathfrak{L}^{[\cdot]} / \mathfrak{T}^{[\cdot]}}\right\}^{\star}
$$

[Symbol $\mathfrak{T}^{\star}$ denotes thermodynamic theory as a whole and symbol $\left\{\mathfrak{Q}_{\mathfrak{L}^{[\cdot]} / \mathfrak{T}^{[\cdot]}}\right\}^{\star}$ is a mark for a transitive and reflexive closure of the set of (any) claims about systems $\left.\mathfrak{L}^{[\cdot]} / / \mathfrak{T}^{[\cdot]}.\right]$.

\& The whole set $\mathfrak{O}_{\mathfrak{L}}^{*}$ of states inaccessible in a given scale of state quantities of the system $\mathfrak{L} / \mathfrak{T}$ along the arbitrary adiabatic path from $l_{\mathfrak{O}_{\mathfrak{L}}}$ in the system $\mathfrak{L}$ (states $\diamond$ ), as well as the set of $\mathfrak{L}$-inaccessible states $\square$ outside this scale, see Figure 1, are considered now to be the thermodynamic bearer of analogy of the semantics of the Gödel's UNDECIDABLE PROPOSITION 17Gen r,

$$
\begin{aligned}
& 17 \text { Gen } r \cong \mathfrak{Q}_{\mathfrak{L}}^{*} {[=\{\{\diamond\} \cup\{\square\}\}] } \\
& \mathfrak{O}_{\mathfrak{L}}^{*}=\left\{\mathfrak{Q}_{\left.\mathfrak{L}^{*}-l_{\mathfrak{Q}_{\mathfrak{L}}}\right\},} \quad \mathfrak{Q}_{\mathfrak{L}}^{*} \varsubsetneqq \mathfrak{Q}_{\mathfrak{L}^{*}} \varsubsetneqq\left\{\mathfrak{Q}_{\mathfrak{L} * / \mathfrak{T}^{*}}\right\}^{\star}\right.
\end{aligned}
$$

- The states from $\mathfrak{O}_{\mathfrak{L}}^{*}$ (from $\left\{\mathfrak{Q}_{\mathfrak{L}^{*}-} l_{\mathfrak{Q}_{\mathrm{L}}}\right\}, \quad\left\{\mathfrak{Q}_{\mathfrak{L}^{*}-} l_{\mathfrak{Q}_{\mathfrak{L}}}\right\}, \quad\left\{\mathfrak{Q}_{\mathfrak{L}^{*}}-l_{\mathfrak{Q}_{\mathfrak{I}^{\prime}}}\right\}, \ldots$, $\left\{\mathfrak{Q}_{\mathfrak{L}^{* *}} l_{\mathfrak{Q}_{\mathfrak{L}}}\right\}, \ldots,\left\{\mathfrak{Q}_{\mathfrak{L}^{* *}} l_{\mathfrak{Q}_{\mathfrak{R}^{*}}}\right\}, \ldots$ ) inaccessible by permitted changes in currently used systems $\mathfrak{L}, \mathfrak{L}^{\prime}, \mathfrak{L}^{+}, \mathfrak{L}^{*}, \ldots$ (within the scale of values of their state quantities and also out of this scale) confirm both existence and properties of

${ }^{12}$ Alternatively Goldbach's conjecture. 
these systems $\mathfrak{L}, \mathfrak{L}^{\prime}, \mathfrak{L}^{+}, \mathfrak{L}^{*}, \ldots$; they confirm adiabacity of changes $l_{\mathfrak{Q}_{\mathfrak{L}}}, l_{\mathfrak{Q}_{\mathfrak{L}^{\prime}}}, l_{\mathfrak{Q}_{\mathfrak{L}}+}, l_{\mathfrak{Q}_{\mathfrak{L}^{*}}}, \ldots$ running in them.

For (to illustrate our analogy) a supposedly countable set of states along the paths $l_{\mathfrak{Q}_{\mathfrak{E}}}$ of changes of the state of the system $\mathfrak{L}$ (for simplicity we can consider the isentrop $l_{2 e}$ only), the PROPOSITION $17 \mathrm{Gen} r$ is a claim of countability set nature, the analog $\mathfrak{O}_{\mathfrak{L}}^{*}$ of which is formulated in the set $\left\{\mathfrak{Q}_{\mathfrak{L} / \mathfrak{T}}\right\}^{\star}$; it as valid that

$$
\begin{aligned}
& \left\{\mathfrak{Q}_{\mathfrak{L} / \mathfrak{T}}\right\}^{\star} \supsetneqq \mathfrak{Q}_{\mathfrak{L}} \quad \text { and } \quad\left\{\mathfrak{Q}_{\mathfrak{L}}-l_{\mathfrak{Q}_{\mathfrak{L}}}\right\} \varsubsetneqq \mathfrak{Q}_{\mathfrak{L}}^{*} \varsubsetneqq\left\{\mathfrak{Q}_{\mathfrak{L} / \mathfrak{T}}\right\}^{\star} \\
& \left\{\mathfrak{Q}_{\mathfrak{L}}-l_{\mathfrak{Q}_{\mathfrak{L}}}\right\} \nsupseteq \mathfrak{Q}_{\mathfrak{L}} \text { and }\left\{\mathfrak{Q}_{\mathfrak{L}}-l_{\mathfrak{Q}_{\mathfrak{L}}}\right\} \nsupseteq l_{\mathfrak{Q}_{\mathfrak{L}}}
\end{aligned}
$$

\subsection{Analogy between Caratheodory and Gödel theorems}

We claim that, II. Caratheodory theorem,

$\checkmark$ if an arbitrary $\boldsymbol{P}$ faff form $\delta Q_{E x t}=\sum_{i=1}^{n} X_{i} \mathrm{~d} x_{i}$, where $X_{i}$ are functions of $n$ variables, continuously differentiable (over a simply continuous domain) has such a quality that in the arbitrary vicinity of arbitrarily chosen fixed point $P$ of the hyperplane $\mathcal{R}\left[P \in \mathcal{R}, \mathcal{R}\left[\left(x_{i}\right)_{i=1}^{n}\right]=C=\right.$ const. $]$ there exists a set of points inaccessible from the point $P$ along the path satisfying the equation $\mathrm{d} Q_{E x t}=0$, then it is possible to find an integration factor for it and then this form is holonomous. In a physical sense and, by means of the Thermodynamics language,

$$
\left(\exists \mid l_{\mathfrak{Q}_{\mathfrak{L}}}\right) \Rightarrow\left(\exists \mid \mathfrak{Q}_{\mathfrak{L}}\right) \Rightarrow\left(\exists \mid \mathfrak{Q}_{\mathfrak{L}}^{*}\right) \Rightarrow\left(\exists \mid \mathfrak{Q}_{\mathfrak{L}^{*}}\right) \Rightarrow\left(\exists \mid\left\{\mathfrak{Q}_{\mathfrak{L} / \mathfrak{T}}\right\}^{\star}\right)
$$

it says what, in its consequence $[w 17 G e n r,(8)]$ and in a meta-arithmeticlogical way, the II. Gödel theorem (corrected semantically by $[3,9,10]$ ) claims;

\& if $\kappa$ is an arbitrary recursive and consistent CLASS OF FORMULAE, then any CLAIM (written as the SENTENCIAL and as such, representing a countable set of claims, which are its implementations) saying that CLASS $\kappa$ is consistent must be constructed outside this set and for this fact it is not $\kappa-P R O V A B L E /$ is $\kappa$ $U N P R O V A B L E$ or cannot be $\kappa-P R O V A B L E$. In fact, it is a part of the inconsistent metasystem $\mathcal{P}^{*}$.

- Outside the consistent system $\mathcal{P}_{\kappa}$, there is a true ("1") formula whose ARITHMETIZATION is $\kappa$-UNPROVABLE FORMULA/PROPOSITION/CLAIM or code $17 \mathrm{Gen} r "{ }^{13}$

. In a physical sense and by the Thermodynamics language,

$$
\left\{\mathfrak{Q}_{\mathfrak{L} / \mathfrak{T}}\right\}^{\star} \nsubseteq \mathfrak{Q}_{\mathfrak{L}^{*}} \nsubseteq \mathfrak{Q}_{\mathfrak{L}}^{*} \nsubseteq \mathfrak{Q}_{\mathfrak{L}} \nsubseteq l_{\mathfrak{Q}_{\mathfrak{L}}}
$$

\& It is possible to claim that, $I$. Caratheodory theorem,

$\checkmark$ if an arbitrary $P$ faff form $\delta Q_{E x t}=\sum_{i=1}^{n} X_{i} \mathrm{~d} x_{i}$ has a integration factor, then there are in the arbitrary vicinity of an arbitrarily chosen fixed point $P$ of the hyperplane $\mathcal{R}$ some points inaccessible from this point $P\left[P \in \mathcal{R}\left[\left(x_{i}\right)_{i=1}^{n}\right]=\right.$ const. $]$ along the path satisfying the equation $\mathrm{d} Q_{E x t}=0$. In a physical sense and by means of the Thermodynamics language,

$$
\left[\mathfrak{Q}_{\mathfrak{L}}^{*} \nsubseteq l_{\mathfrak{Q}_{\mathfrak{L}}}\right] \wedge\left[\left(\left\{\mathfrak{Q}_{\mathfrak{L} / \mathfrak{T}}\right\}^{\star}-\mathfrak{Q}_{\mathfrak{L}}^{*}\right) \nsubseteq l_{\mathfrak{Q}_{\mathrm{L}}}\right]
$$

\footnotetext{
13 Any attempt to prove/TO PROVE it (to infer/TO INFER it) within the system $\mathcal{P}_{k}$ assumes or leads to the Circulus Viciosus.
} 
it says what, in a meta-arithmetic-logical way, the $I$. Gödel theorem (corrected semantically by $[3,9,10])$ claims;

\& for every recursive and consistent CLASS OF FORMULAE $\kappa$ and outside this set, there is such a true ("1") CLAIM $r$ with free VARIABLE $v[r \triangleq r(v)]$ that neither PROPOSITION $v$ Gen $r$ nor PROPOSITION Neg $(v$ Gen $r)$ belongs to the set Flg $(\kappa)$,

$$
[v \operatorname{Gen} r \notin F l g(\kappa)] \&[\operatorname{Neg}(v \operatorname{Gen} r) \notin F l g(\kappa)]
$$

FORMULA $v$ Gen $r$ and Neg $(v G e n r)$ are not $\kappa$-PROVABLE_FORMULA $v$ Gen $r$ is not $\kappa$-DECIDABLE. They are elements of inconsistent (meta)system $\mathcal{P}^{*}$.

\& For us, as an isolated system $\mathfrak{L}$, to achieve such a "state," it is necessary to consider the states with values of state quantities which are not a part of the domain of solution of the state equation for $\mathfrak{L}$. The system $\mathfrak{L}$ has not been designed for them (so, we are facing inconsistency). For example, the required volume $V$ and temperature $T$ should be greater than their maxima $V_{\max }$ and $T_{\max }$ achievable by the system $\mathfrak{L}$. In order "to achieve" them, the system $\mathfrak{L}$ itself would have to "get out of itself," and in order to obtain values $V$ and $T$ greater than $V_{\max }$ and $T_{\max }$, it would have to "redesign"/reconstruct itself. However, it is us, being in a position of the hierarchically higher object, who has to do so, from the outside the state space $\mathfrak{Q}_{\mathfrak{L} / \mathfrak{T}}$ (from the outside the volume $V_{\max }$ ), which the system may occupy now. ${ }^{14}$

- This "procedure" corresponds to the CLAIM/PROPOSITION/FORMULA 17Gen $r$ construction by means of (Cantor's) diagonal argument and Caratheodory proof.

\& The states unachievable within the state spaces of the systems $\mathfrak{L}, \mathfrak{L}^{\prime}, \mathfrak{L}^{+}, \mathfrak{L}^{*}, \ldots$ or inaccessible from them are creating, as a whole, a certain

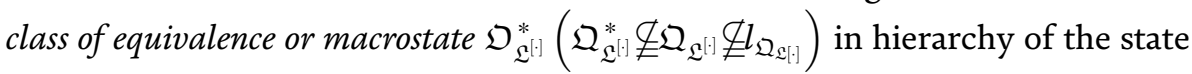
spaces, from the point of their possible development, of always superior systems $-\left\{\mathfrak{L}^{\prime} \cup \mathfrak{L}^{+} \cup \mathfrak{L}^{*}\right\}$ for $\mathfrak{L},\left\{\mathfrak{L}^{+} \cup \mathfrak{L}^{*}\right\}$ for $\mathfrak{L}^{\prime}, \mathfrak{L}^{*}$ for $\mathfrak{L}^{+}, \ldots$. The existence of the macrostate $\mathfrak{O}_{\mathfrak{L}[\cdot]}^{*}$, already beginning from the original system $\mathfrak{L}$ (macrostate $\mathfrak{O}_{\mathfrak{L}}^{*}$ ), confirms the existence of the currently considered (adiabatic) system $\mathfrak{L}^{[\cdot]}$ and its properties, especially its adiabacity. And by this, in our analogy, it also con rms the consistency of its arithmetic/mathematical analog $\mathcal{P}, \mathcal{P}^{\prime}, \mathcal{P}^{+}, \ldots$ (a complement of the set cannot exist without this set) and, on the contrary,

$$
\begin{aligned}
& \left(\exists \mid\left\{\mathfrak{Q}_{\mathfrak{L} / \mathfrak{T}}\right\}^{\star}\right) \Rightarrow\left(\exists \mid \mathfrak{Q}_{\mathfrak{L}^{\mid}}^{*}\right), \quad\left(\exists \mid\left\{\mathfrak{Q}_{\mathfrak{L} / \mathfrak{T}}\right\}^{\star}\right) \Leftarrow\left(\exists \mid \mathfrak{Q}_{\mathfrak{L}^{\mid !}}^{*}\right) ; \\
& {\left[\left[\left(\exists \mid \mathfrak{Q}_{\mathfrak{L}^{[!]}}^{*}\right) \Rightarrow\left(\exists \mid \mathfrak{L}^{[\cdot]}\right)\right] \&\left[\left(\exists \mid \mathfrak{L}^{[\cdot]}\right) \Rightarrow\left(\exists \mid \mathfrak{Q}_{\mathfrak{L}^{[]]}}^{*}\right)\right]\right]} \\
& {\left[\left(\exists \mid \mathfrak{Q}_{\mathfrak{L}^{[]}}^{*]}\right) \Leftrightarrow\left(\exists \mid \mathfrak{L}^{[\cdot]}\right)\right]}
\end{aligned}
$$

- Based just upon this point of view, we assign the set/macrostate or equivalence class $\mathfrak{O}_{\mathcal{L}^{[]]}}^{*}$ the meaning of the bearer of the sense of the Gödel's UNDECIDABLE PROPOSITION $17 \mathrm{Gen} r$ for $\mathcal{P}$,

\footnotetext{
14 This also involves introduction of the representative $\theta_{0}$ of Fermat's Last Theorem provided we are speaking about $\mathfrak{L}$ with $l_{\mathfrak{O}_{\mathfrak{L}}}$ and provided we require enlargement $\mathfrak{L}^{\prime}$ in order to get $\mathfrak{L}^{\prime} \cong \mathcal{P}^{\prime}$.

The specific states accessible in the state space $\mathfrak{O}_{\mathfrak{L}}=\left\{p \in\left\langle p_{\text {min }}, p_{\text {max }}\right\rangle, V \in\left\langle V_{\text {min }}, V_{\text {max }}\right\rangle\right.$,

$T \in\left\langle T_{\min }, T_{\max }\right\rangle / U \in\left\langle U_{\min }, U_{\max }\right\rangle, \ldots$ of the isolated system $\mathfrak{L}$ through reversible or irreversible changes other than adiabatic are thermodynamic analogy (interpretation) of the enlargement of the axiomatics of the original system $\mathcal{P}_{[\kappa]}$ to the new system $\mathcal{P}^{\prime}, \mathcal{P}^{+}, \ldots$, similar/relative to the $\mathcal{P}_{[\kappa]}$. Such an enlargement of the system $\mathcal{P}$ to a certain system $\mathcal{P}^{[\cdot]}$ enabled Andrew Wiles to prove the Fermat's Last Theorem. Through its representative $\theta_{0}$ we enlarge $\mathfrak{L}$ to $\mathfrak{L}^{\prime}, \mathfrak{L}^{\prime} \cong \mathcal{P}^{\prime}$.
} 
- the $\mathfrak{L}$-unachievability of the set $\mathfrak{O}_{\mathcal{L}^{[j]}}^{*}$ is in the position of the analog for this, in fact, methodological axiom which has been formulated in a certain hierarchically higher inferential (meta)system $\mathcal{P}^{*}, \mathcal{P}^{*} \cong\left\{\mathfrak{O}_{\mathfrak{L}}\right\}^{\star}$. In accordance with the above and with Figure 1, we write for $\mathfrak{L} / \mathcal{P}$

$$
\begin{aligned}
\boldsymbol{\theta}_{4} \in\left\{\mathfrak{Q}_{\mathfrak{L}^{*}} l_{\mathfrak{Q}_{\mathfrak{L}}}\right\}=\{\{\diamond\} \cup\{\square\}\}=\mathfrak{Q}_{\mathfrak{L}}^{*} \varsubsetneqq\left\{\left\{\mathfrak{Q}_{\mathfrak{L}^{*}}\right\}^{\star}-l_{\mathfrak{Q}_{\mathfrak{L}}}\right\} \varsubsetneqq\left\{\mathfrak{Q}_{\mathfrak{L}}\right\}^{\star} \\
{\left[l_{\mathfrak{Q}_{\mathfrak{L}}} \nvdash \boldsymbol{\theta}_{4}\right] \Rightarrow\left[l_{\mathfrak{Q}_{\mathfrak{L}}} \nvdash\left\{\mathfrak{Q}_{\mathfrak{L} *}-l_{\mathfrak{Q}_{\mathfrak{L}}}\right\}\right] \quad\left[\in\left\{\left\{\mathfrak{Q}_{\mathfrak{L} *}\right\}^{\star}-l_{\mathfrak{Q}_{\mathfrak{L}}}\right\}\right] } \\
{\left[l_{\mathfrak{Q}_{\mathfrak{L}}} \nvdash\left\{\mathfrak{Q}_{\mathfrak{L} *}-l_{\mathfrak{Q}_{\mathfrak{L}}}\right\}\right] \cong\left[l_{\mathfrak{Q}_{\mathfrak{L}}} \nsubseteq\left\{\mathfrak{Q}_{\mathfrak{L} *}-l_{\mathfrak{Q}_{\mathfrak{L}}}\right\}\right] } \\
{\left[l_{\mathfrak{Q}_{\mathfrak{L}}} \nvdash\left[l_{\mathfrak{Q}_{\mathfrak{L}}} \nvdash\left\{\mathfrak{Q}_{\mathfrak{L} *}-l_{\mathfrak{Q}_{\mathfrak{L}}}\right\}\right]\right] \quad\left[\in\left\{\left\{\mathfrak{Q}_{\mathfrak{L} *}\right\}^{\star}-l_{\mathfrak{Q}_{\mathfrak{L}}}\right\}\right] } \\
{\left[l_{\mathfrak{Q}_{\mathfrak{L}}} \nvdash\left[l_{\mathfrak{Q}_{\mathfrak{L}}} \nvdash\left[l_{\mathfrak{Q}_{\mathfrak{L}}} \nvdash\left\{\mathfrak{Q}_{\mathfrak{L} *}-l_{\mathfrak{Q}_{\mathfrak{L}}}\right\}\right]\right]\right] \quad\left[\in\left\{\left\{\mathfrak{Q}_{\mathfrak{L} *}\right\}^{\star}-l_{\mathfrak{Q}_{\mathfrak{L}}}\right\}\right], \ldots }
\end{aligned}
$$

and further, for the theory $l_{\mathfrak{O}_{\mathfrak{L}}} / \mathcal{T}_{\mathcal{P A}}$, following (1)-(6) and [4], we write

$$
\begin{aligned}
& l_{\mathfrak{Q}_{\mathfrak{L}}} \cong \mathcal{T}_{P \mathcal{A}}, \quad \text { card } l_{\mathfrak{Q}_{\mathfrak{L}}}=\operatorname{card} \mathcal{T}_{\mathcal{P}_{\mathcal{A}}}=\aleph_{0} \\
& \operatorname{card}\left\{\mathfrak{Q}_{\mathfrak{L} *}\right\}^{\star}=\aleph_{1}, \quad \operatorname{card}\left\{\left\{\mathfrak{Q}_{\mathfrak{L} *}\right\}^{\star}-l_{\mathfrak{Q}_{\mathfrak{L}}}\right\}=1 \\
& l_{\mathfrak{Q}_{\mathfrak{L}}} \cong 17, \quad\{\{\diamond\} \cup\{\square\}\} \cong 19\left[19 \in\left\{\left\{\mathfrak{Q}_{\mathfrak{L}^{*}}\right\}^{\star}-l_{\mathfrak{Q}_{\mathfrak{L}}}\right\}\right] \\
& y=q[17,19] \cong\left[\boldsymbol{\theta}_{[\cdot]} \nvdash\{\{\diamond\} \cup\{\square\}\}\right], \quad p=17 \text { Gen } q[17,19] \\
& y[Z(y)]=q[17, \quad y] \cong\left[\boldsymbol{\theta}_{[\cdot]} \nvdash\left[\boldsymbol{\theta}_{[\cdot]} \nvdash\{\{\diamond\} \cup\{\square\}\}\right]\right] \\
& {\left[\left[\boldsymbol{\theta}_{[\cdot]} \nvdash(\boldsymbol{\theta} \cup \square)\right] \in\{\{\boldsymbol{\theta}\} \cup\{\square\}\}^{\star}\right]} \\
& {\left[\boldsymbol{\theta}_{[\cdot]} \nvdash\left[\boldsymbol{\theta}_{[\cdot]} \nvdash\{\{\diamond\} \cup\{\square\}\}\right]\right] \quad\left[\in\{\{\diamond\} \cup\{\square\}\}^{\star}\right]} \\
& {\left[\boldsymbol{\theta}_{[\cdot]} \nvdash\left[\boldsymbol{\theta}_{[\cdot]} \nvdash\left[\boldsymbol{\theta}_{[\cdot]} \nvdash\{\{\diamond\} \cup\{\square\}\}\right]\right]\right], \ldots}
\end{aligned}
$$

For 19: $=Z(p)$ is $p[Z(p)]=r(17)$ and $r(17) \cong\{\{\diamond\} \cup\{\square\}\}^{\star}$ and so we can write neatly

$$
\begin{aligned}
& {\left[\forall_{\boldsymbol{\theta}_{[]} \in l_{\mathfrak{Q}_{\mathfrak{L}}}}\right] \cong 17 G e n, \quad\left[\forall_{\boldsymbol{\theta}_{[\}]} \in l_{\mathfrak{Q}_{\mathfrak{L}}}} \mid\left[\boldsymbol{\theta}_{[\cdot]} \nvdash\{\{\diamond\} \cup\{\square\}\}\right]\right] \cong[17 \operatorname{Gen}[q(17,19)]]}
\end{aligned}
$$

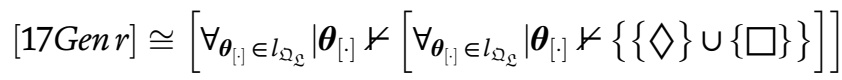

$$
\begin{aligned}
& {[17 G e n r] \cong\left[l_{\mathfrak{Q}_{\mathfrak{L}}} \nvdash \quad\left[l_{\mathfrak{Q}_{\mathfrak{L}}} \nvdash\left\{\mathfrak{O}_{\mathfrak{L}^{*}}-l_{\mathfrak{Q}_{\mathfrak{L}}}\right\}\right]\right], \ldots}
\end{aligned}
$$

which is the same as (21).

- It is obvious from our thermodynamic analogy that CLAIM/PROPOSITION 17 Gen $r$ for has to be true and in connection with Gödel's II. theorem, and in accordance with Caratheodory we claim that

$$
\mathfrak{Q}_{\mathfrak{L}[\cdot]}^{*} \cong 17 G e n r \text { for } \mathfrak{L} / \mathcal{P} \quad\left[v G e n r \text { for } \mathfrak{L}^{\prime} / \mathcal{P}^{\prime}, \mathfrak{L}^{+}, \ldots\right]
$$

\& The notation $17 G e n r$ itself expresses the property of the system $\mathcal{P}$ and also the theory $\mathcal{T}_{\mathcal{P A}_{\mathcal{A}}}$, just as an subject which itself is not and cannot be the object of its own, and thus its notation is not and cannot be one of the objects of the system $\mathcal{P}$ [similarly, as (17) is valid, $\mathfrak{O}_{\mathfrak{L}}^{*} \nsubseteq \mathfrak{Q}_{\mathfrak{L}} \nsubseteq l_{\mathfrak{Q}_{\mathfrak{l}}}$ ]. 
Demonstration: Following (8) $[\operatorname{Wid}(\mathcal{P}) \Rightarrow 17$ Gen $r]$, we claim for the systems $\mathfrak{L} / \mathcal{P}$ that

$$
\begin{aligned}
& {\left[\mathrm{d} Q_{\text {Ext }}^{\mathfrak{L}}=0\right] \cong w, \quad\left[\mathrm{~d} Q_{E x t}^{\mathfrak{L}}=0\right] \equiv \mathfrak{L} ; \quad w \cong\left[\mathrm{d} Q_{\text {Ext }}^{\mathfrak{L}}=0\right], \quad \mathfrak{L} \equiv\left[\mathrm{d} Q_{\text {Ext }}^{\mathfrak{L}}=0\right]} \\
& (\exists \mid \mathfrak{L}) \Rightarrow\left(\exists \mid \mathfrak{O}_{\mathfrak{L}}^{*}\right),\left(\exists \mid \mathfrak{Q}_{\mathfrak{L}}^{*}\right) \cong 17 G e n r ; \quad\left(\exists \mid \mathfrak{O}_{\mathfrak{L}}^{*}\right) \Rightarrow(\exists \mid \mathfrak{L}),(\exists \mid \mathfrak{L}) \cong 17 G e n r \\
& {\left[(\exists \mid \mathfrak{L}) \Rightarrow\left(\exists \mid \mathfrak{O}_{\mathfrak{L}}^{*}\right)\right] \cong[w \Rightarrow(17 G e n r)] ; \quad\left[\left(\exists \mid \mathfrak{O}_{\mathfrak{L}}^{*}\right) \Rightarrow(\exists \mid \mathfrak{L})\right] \cong[(17 G e n r) \Rightarrow w]} \\
& \text { so, that } \quad\left[\left[\left(\exists \mid \mathfrak{O}_{\mathfrak{L}}^{*}\right) \Rightarrow(\exists \mid \mathfrak{L})\right] \cong[(17 G e n r) \Rightarrow w]\right] \\
& \& \\
& {\left[\left[(\exists \mid \mathfrak{L}) \Rightarrow\left(\exists \mid \mathfrak{O}_{\mathfrak{L}}^{*}\right)\right] \cong[w \Rightarrow(17 \text { Gen } r)]\right] \text { and then }} \\
& \left(\exists \mid \mathfrak{O}_{\mathfrak{L}}^{*}\right) \equiv 17 \text { Genr }
\end{aligned}
$$

I. Gödel theorem (corrected semantically by $[3,9,10]$ ):

For every recursive and consistent CLASS OF FORMULAE $\kappa$, and outside this set, there exists the true (" 1 ") CLAIM $\mathrm{r}$ with a free VARIABLE $\mathrm{v}$ that neither the $C L A I M$ vGen r nor the CLAIM Neg(vGen r) belongs to the set $\mathrm{Flg}(\kappa)$

$$
[v \operatorname{Gen} r \notin \mathrm{Flg}(\kappa)] \&[\operatorname{Neg}(v \operatorname{Gen} r) \notin / F \lg (\kappa)],
$$

$C L A I M S$ vGen $\mathrm{r}$ and Neg(vGen $\mathrm{r}$ ) are not $\kappa-P R O V A B L E$, the CLAIM vGen $\mathrm{r}$ is not $\kappa$-DECIDABLE.

[They are elements of the formulating/syntactic metasystem $\kappa^{\star}$, inconsistent against $\kappa$ ].

II. Gödel theorem (corrected semantically by $[3,9,10])$ :

If $\kappa$ is an arbitrary recursive and consistent CLASS OF FORMULAE, then any CLAIM saying that CLASS $\kappa$ is consistent must be constructed outside this set and for this fact, it is not $\kappa-P R O V A B L E$.

The consistency of the CLASS OF FORMULAE $\kappa$ is tested by the relation Wid $(\kappa)$.

$$
\operatorname{Wid}(\kappa) \sim(\operatorname{Ex})\left[C L A I M(\mathbf{x}) \& \overline{\operatorname{Proof}_{\kappa}(\mathbf{x})}\right]
$$

The FORMULAE class $\kappa$ is consistent.

$\Leftrightarrow$

at least one $\kappa$-UNPROVABLE CLAIM x exists.

Now $\mathbf{x}=17$ Gen $\mathbf{r} \notin \mathcal{P} / \mathcal{T}_{\mathcal{P A}}, \kappa=\mathcal{T}_{\mathcal{P A}}, \mathcal{T}_{\mathcal{P A}} \subset \mathcal{P} \subset \mathcal{P}^{\star}$

Then, semantically understood and with the language of logic and metaarithmetics, the full meaning of the Gödel proof expresses the universal validity of the II. Law of Thermodynamics. ${ }^{15}$

$$
\left[I I . P . T .\left[\text { Proof }_{\mathcal{P}}(17 \text { Gen } \mathbf{r})=" 0 "\right]=" 1 "\right]=\operatorname{Wid}\left(\mathcal{T}_{\mathcal{P}, A}\right)
$$

\footnotetext{
15 Our consideration is based on the similarity between the Cantor diagonal argument used in construction of the Gödel Undecidable Formula and the proof way of the Caratheodory theorems; adiabacity/consistency is prooved by leaving them and sustaining their validity - paradox.
} 


\section{Conclusion}

Peano Arithmetic theory is generated by its inferential rules (rules of the inferential system in which it is formulated). It consists of parts bound mutually just by these rules, but none of them is not identical with it nor with the system in their totality.

By information-thermodynamic and computing analysis of Peano arithmetic proving, we have showed why the Gödel formula and its negation are not provable and decidable within it. They are constructed, not inferred, by the diagonal argument, which is not from the set of the inferential rules of the system. The attempt to prove them leads to awaiting of the end of the infinite cycle being generated by the application of the substitution function just by the diagonal argument. For this case, the substitution function is not countable and for this it is not recursive (although in the Gödel original definition is claimed that it is). We redefine it to be total by the zero value for this case. This new substitution function generates the Gödel numbers of chains, which are not only satisfying the recursive grammar of formulae but it itself is recursive. The option of the zero value follows also from the vision of the inferential process as it would be the information transfer. The attempt to prove the Gödel Undecidable Formula is the attempt of the transfer of that information, which is equal to the information expressing the inner structure of the information transfer channel. In the thermodynamic point of view, we achieve the equilibrium status, which is an equivalent to the inconsistent theory. So, we can see that the Gödel Undecidable Formula is not a formula of the Peano Arithmetics and, also, that it is not an arithmetical claim at all. From the thermodynamic consideration follows that even we need a certain effort or energy to construct it, within the frame of the theory this is irrelevant. It is the error in the inference and cannot be part of the theory and also it is not the system. Its information value in it (as in the system of the information transfer) is zero. But it is the true claim about inferential properties of the theory (in fact, of the properties of the information transfer).

Any description of real objects, no matter how precise, is only a model of them, of their properties and relations, making them available in a specified and somewhat limited (compared with the reality) point of view determined by the description/ model designer. This determination is expressed in definitions and axiomatics of this description/model/theory_-both with definitions and by axioms and their number. Hence, realistically/empirically or rationally, it will also be true about (objects of) reality what such a model, called recursive and able-of-axiomatization, does not include. With regard of reality any such a model is axiomatically incomplete, even if the system of axioms is complete. In addition, and more importantly, this description/model of objects, of their properties and possible relations (the theory about reality) cannot include a description of itself just as the object of reality defined by itself (any such theory/object is not a subject of a direct description of itself). The description/model or the theory about reality is a grammar construction with substitutes and axiomatization and, as such, it is incomplete in the Gödelian way-the grammar itself does not prevent a semantical mixing; but any observed real object cannot be the subject of observation of itself and this is valid for the considered theory, just as for the object of reality, too. No description of reality arranged from its inside or created within the theory of this reality can capture the reality completely in wholeness of its all own properties. It is impossible for the models/theories considered, independently on their axiomatization. They are limited in principle [in the real sense of the Gödel theorems (in the Gödelian way)].

Now, with our better comprehension, we can claim that the consistency of the recursive and axiomatizable system can never be proved in it itself, 
even if the system is consistent really. The reason is that a claim of the consistency of such a system is designable only if the system is the object of outer observation/measuring/studies, which is not possible within the system itself. Ignoring this approach is also the reason for the formulation of the Gibbs paradox and Halting Problem. Also, our awareness of this fact results in our full understanding of the meaning and proof of the Gödel theorems, very often explained and described incomprehensibly, even inconsistently or paradoxically, and which is parallel with the way of the Caratheodory proof of the II. Thermodynamic Principle.

Vienna Circle, 1931-1935, logical positivists Rudolph Carnap
and Otto von Neurath:
"Any scientifically meaningful statement is expressible in physical terms,
about a movement in the observable space and time or, if the statement is not
expressible this way it is meaningful scientifically when it is convertible to a
statement about a language, otherwise it is of no scientific meaning."

\section{Acknowledgements}

Supported by the grant of Ministry of Education of the Czech Republic MSM 6046137307.

Many thanks are to be expressed to my brother Ing. Petr Hejna for his help with English language and formulations of both this and all the previous texts.

\section{A. Appendix}

\section{A.1 Summarizing comparison}

\& Under the adiabacity, $[\mathrm{d}] \mathrm{Q}_{\mathrm{Ext}}=0$, of the system $\mathfrak{L}$, it is not possible to derive such a CLAIM that is stating this adiabatic supposition. This CLAIM is constructible not adiabatically, outside the adiabatic $\mathfrak{L}$ only.

\& Under the consistency of the system $\mathcal{P}$, it is not possible to derive such a CLAIM that is stating this consistency supposition. This CLAIM is constructible purely syntactically, outside the consistent $\mathcal{P}$ only (in $\mathcal{P}^{*}-\mathcal{P}$ ) (Figure A1).

\& Without $\mathcal{P}^{*}$ we could not know that $\mathbf{P}$ is not self-referencing and is consistent.

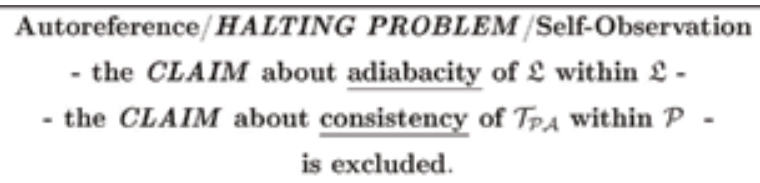

This is the nature law expressed by the Caratheodory form of the II. P.T. and by the Gödel theorems' sense.

\section{The eye can not look at and into itself.}

Any mixing of the various observation/expressing/approach levels leads to the paradoxes and is to be excluded from the cognitive thinking. 


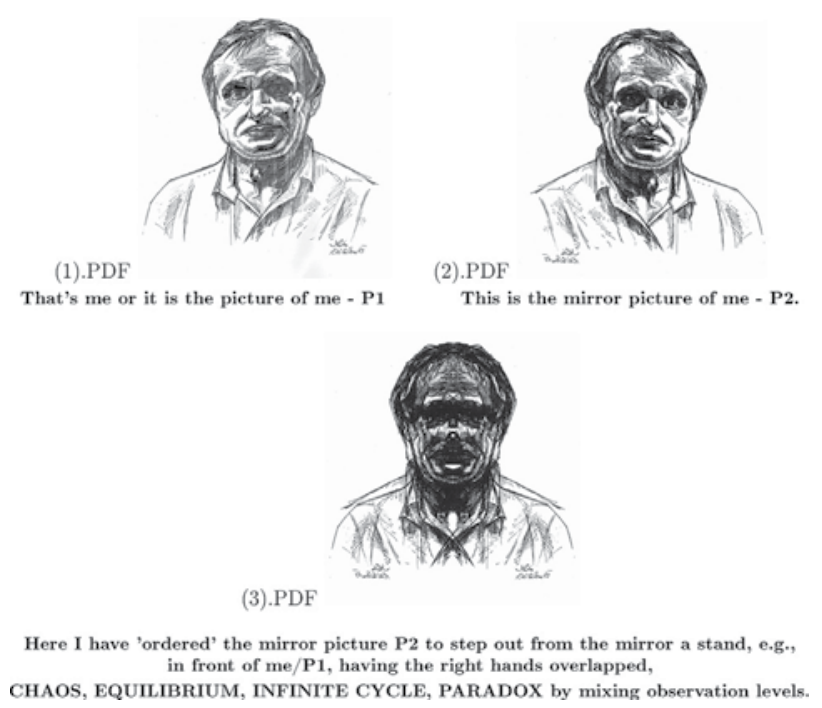

Figure A1.

Example of not distinguishing the reality and its image.

\section{A.2 The proof way of Caratheodory theorems}

$I$. Let the form $\delta Q=\sum_{i=1}^{n} X_{i} x_{i}$ has the integration factor $v$ and let $d \mathcal{R}=\sum_{i=1}^{n} \frac{1}{v} X_{i} \mathrm{~d} x_{i}$. Then the Pfaff equation $\delta Q=\sum_{i=1}^{n} X_{i} \mathrm{~d} x_{i}=0$ has the solution in the form $\mathcal{R}\left(x_{1}, \ldots, x_{k}\right)=$ const. and this solution represents a family of hyperplanes in $n$-dimensional space, not intersecting each other. Let us pick now the point $P\left(x_{1}^{0}, \ldots, x_{n}^{0}\right)$ determined by our choice of const. $=C$. Only the points lying in the hyperplane $\mathcal{R}\left(x_{1}^{0}, \ldots, x_{n}^{0}\right)$ are accessible from the point $P$ along the path satisfying the condition $\mathrm{d} Q=0$. All the points not lying in this hyperplane are inaccessible from the point $P$ along the path satisfying the condition $\mathrm{d} Q=0$ (Figure A2).

$I I$. Let us pick the point $V$, e.g., from $\mathbb{R}^{3}$, lying in a vicinity of the point $P$, which is not accessible from $P$ following the path $\mathrm{d} Q=0$. Let $g$ be a line going through the point $P$ and let $g$ be oriented $(\vec{g})$ in such way that it does not satisfy the condition $\mathrm{d} Q=0$. The point $V$ and the line $g$ determine a plane $X_{i}=X_{i}(u, v), i=1,2$, 3. Let us
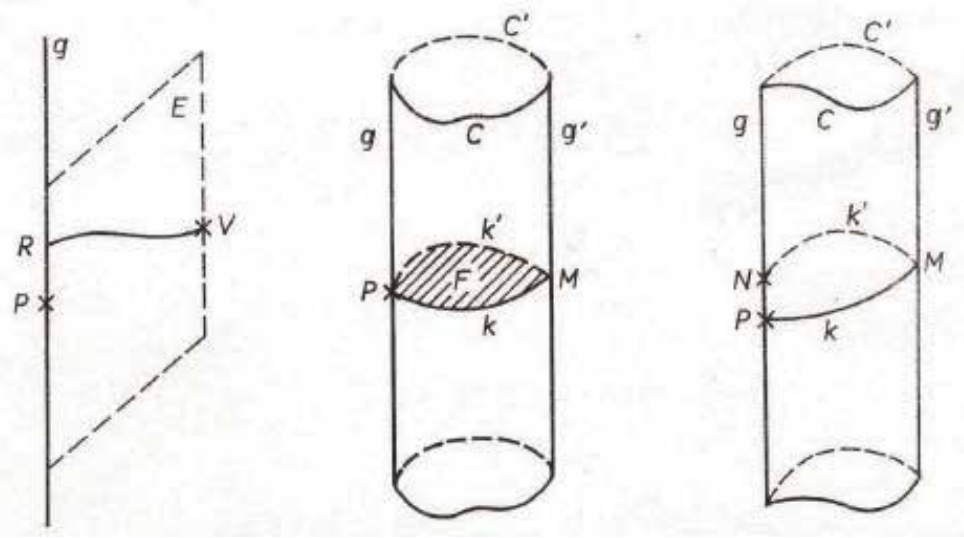

Figure A2.

The proof way of the Caratheodory theorems. 
consider a curve $k$ in this plane, going through the point $V\left(u_{0}, v_{0}\right)$ in that way $(\vec{g})$ that $\mathrm{d} Q=0$ is supposedly valid along this curve. There is only one curve $k$ for the point $V\left(u_{0}, v_{0}\right)$. It lies in our plane, the plane $X_{i}=X_{i}(u, v)$, and then it is valid for it $\mathrm{d} X_{i}=\frac{\partial X_{i}}{\partial u} \mathrm{~d} u+\frac{\partial X_{i}}{\partial v} \mathrm{~d} v$ and, considering $\mathrm{d} Q=0$ along $k$, we get $\sum_{i=1}^{3} X_{i} \frac{\partial X_{i}}{\partial u} \mathrm{~d} u+$ $\sum_{i=1}^{3} X_{i} \frac{\partial X_{i}}{\partial v} \mathrm{~d} v=0$.

The curve $k$, however, intersects the line $g$ in the point $R$, which is inaccessible from the point $P$ along the path with $\mathrm{d} Q=0$ (for $\left.\mathrm{d} Q_{R_{\vec{g}}} \neq 0\right)$. Otherwise, the point $V$ would also be accessible from the point $P$ through $R$ and $k\left(\mathrm{~d} Q_{R_{k}}=0\right)$, which is a conflict with the original assumption. By a suitable selection of $V$, it is possible to have the point $R$ arbitrarily close to the point $P$; in the arbitrary vicinity of the point $P$, there are points inaccessible from the point $P$ along the path with $\mathrm{d} Q=0$. Now, let us pick a line $g^{\prime}$ parallel to the line $g$, and a cylinder $C$ going through these two lines. We consider that the curve $k$ satisfying the relation $\mathrm{d} Q=0$ is on this cylinder $C^{\prime}$ goes through the point $P$ and intersects the line $g^{\prime}$ in the point $M$.

Now, let us consider another cylinder $C^{\prime}$ as the continuation of $C$ with $g^{\prime}$ and $g$. Let us use the symbol $k^{\prime}$ for the continuation of the curve $k$ in $C^{\prime}$. Then the curve $k^{\prime}$ must intersect the line $g$ in the point $P$. Otherwise, it would be possible to deform the plane $C^{\prime}$ as much as to get $C$, thus continually merging the intersecting point $N$ into the point $P$ and at the moments of discrepancy of the points $P$ and $N$, it would be possible to reach the point $P$ from the point $N$ along the line $g$ (supposedly with $\mathrm{d} Q=0)$. However, the condition $\mathrm{d} Q=0$ is not valid there $\left(\mathrm{d} Q_{R_{\vec{g}}} \neq 0\right)$. By deforming $C^{\prime}$ into $C$, the $k$ and $k^{\prime}$ would close a plane $F$ where $\mathrm{d} Q=0$. If the equation of this plane has the form $\mathcal{R}\left(x_{i}\right)_{i=1}^{3}=$ const., then the equation $\mathrm{d} Q=0$ has a solution-an integration factor for the Pfaff form $\delta Q=\sum_{i=1}^{3} X_{i} \mathrm{~d} x_{i}$ exists [11].

\section{A.3 Information thermodynamic concept removing autoreference}

The concept for ceasing the autoreference, based on the two Carnot Cycles disconnected as for their heaters and described informationally, shows the following Figure A3. (also see $[1,2,4]$ ):

For $\Delta A$, it is valid in the cycle $\mathcal{O}^{\prime \prime}$ that

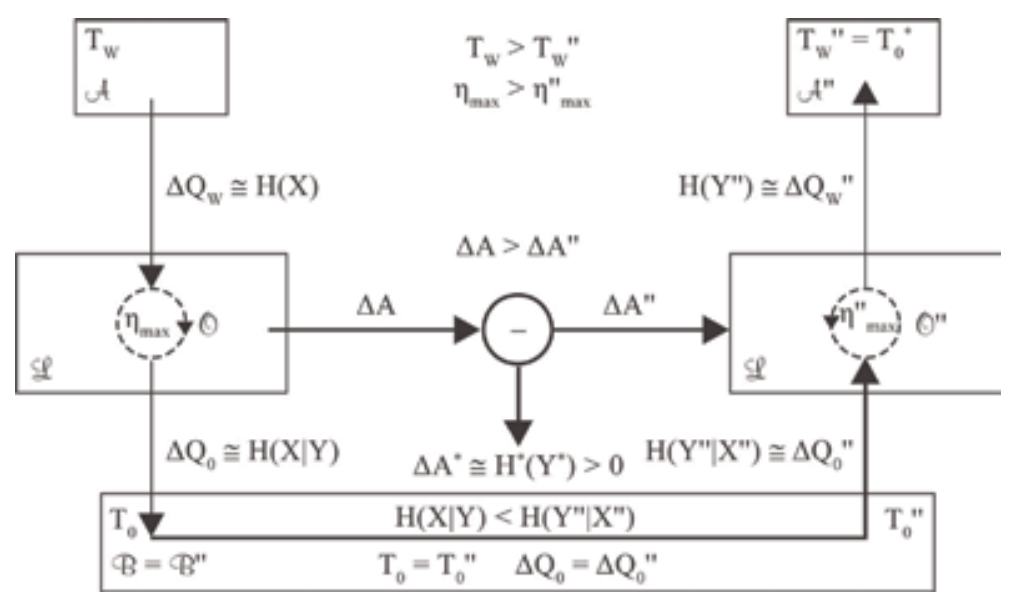

Figure A3.

The concept for ceasing the autoreference. 


$$
\begin{aligned}
\Delta A^{\prime \prime} & =\Delta Q_{W}^{\prime \prime} \cdot\left(1-\frac{T_{0}}{T_{W}^{\prime \prime}}\right)=\Delta Q_{W} \cdot \frac{T_{W}^{\prime \prime}}{T_{W}} \cdot\left(1-\frac{T_{0}}{T_{W}^{\prime \prime}}\right)= \\
& =\Delta Q_{W} \cdot\left(\frac{T_{W}^{\prime \prime}}{T_{W}}-\frac{T_{0}}{T_{W}}\right)=\mathrm{k} \cdot H(X) \cdot\left(T_{W}^{\prime \prime}-T_{0}\right) \\
& =\mathrm{k} \cdot H(X) \cdot T_{W}^{\prime \prime}\left(1-\frac{T_{0}}{T_{W}^{\prime}}\right)=\mathrm{k} \cdot H(X) \cdot T_{W}^{\prime \prime}\left(1-\beta^{\prime \prime}\right)=\mathrm{k} \cdot T^{\prime \prime}{ }_{W} \cdot H\left(Y^{\prime \prime}\right)
\end{aligned}
$$

and, further, for $\Delta A$ in the cycle $\mathcal{O}$, we have

$$
\Delta A=\mathrm{k} \cdot H(X) \cdot T_{W}(1-\beta)=\mathrm{k} \cdot H(X) \cdot T_{W}\left(1-\frac{T_{0}}{T_{W}}\right)
$$

and thus, for the cycles $\mathcal{O}^{\prime \prime}$ and $\mathcal{O}$, it is valid that

$$
\begin{gathered}
\frac{\Delta A^{\prime \prime}}{\mathrm{k} T_{W}^{\prime \prime}}=H(X) \cdot\left(1-\frac{T_{0}}{T_{W}^{\prime \prime}}\right)=H(X) \cdot\left(1-\beta^{\prime \prime}\right)=H(X) \cdot \eta_{\max }^{\prime \prime} \\
\frac{\Delta A}{\mathrm{k} T_{W}}=H(X) \cdot\left(1-\frac{T_{0}}{T_{W}}\right)=H(X) \cdot(1-\beta)=H(X) \cdot \eta_{\max }
\end{gathered}
$$

For the whole work $\Delta A^{*}$ of the combined cycle $\mathcal{O O}^{\prime \prime}$, we have

$$
\Delta A^{*}=\Delta A-\Delta A^{\prime \prime}=\left[\mathrm{k} T_{W} \cdot H(X) \cdot(1-\beta)-k{T^{\prime \prime}}_{W} \cdot H(X) \cdot\left(1-\beta^{\prime \prime}\right)\right]>0
$$

Then, for the whole change of the thermodynamic entropy within the combined cycle $\mathcal{O O}^{\prime \prime}$ (measured in information units Hartley, nat, bit) and thus for the change of the whole information entropy $H^{*}\left(Y^{*}\right)$, it is valid that

$$
\begin{aligned}
H^{*}\left(Y^{*}\right) & =\frac{\Delta A^{*}}{\mathrm{k} T_{W}}=H(X) \cdot\left[(1-\beta)-\frac{T_{W}^{\prime \prime}}{T_{W}} \cdot\left(1-\beta^{\prime}\right)\right] \\
& =H(X) \cdot\left(1-\frac{T_{0}}{T_{W}}-\frac{T_{W}^{\prime \prime}}{T_{W}}+\frac{T_{0}}{T_{W}}\right)=H(X) \cdot\left(1-\frac{T_{W}^{\prime \prime}}{T_{W}}\right)
\end{aligned}
$$

It is valid, for $\Delta A^{*}$ is a residuum work after the work $\Delta A$ has been performed at the temperature $T_{W}$. Evidently, the sense of the symbol $T_{W}^{\prime \prime}$ (within the double cycle $\mathcal{O O}^{\prime \prime}$ and when $\Delta Q_{0}=\Delta Q_{0}$ ) is expressible by the symbol $T^{*}{ }_{0}$, which is possible, for the working temperatures of the whole cycle $\mathcal{O O}^{\prime \prime}$ are $T_{W}$ and $T_{W}=T^{*}{ }_{0}$. The relation (30) expresses that fact that the double cycle $\mathcal{O O}^{\prime \prime}$ is the direct Carnot Cycle just with its working temperatures $T_{W}>T_{W}=T^{*}$. In the double cycle $\mathcal{O O}^{\prime \prime}$, it is valid that

$$
\begin{gathered}
\beta^{\prime \prime}=\frac{\Delta Q_{0}^{\prime \prime}}{\Delta Q_{W}^{\prime \prime}}=\frac{\frac{\Delta Q_{0}^{\prime \prime}}{T_{W}^{\prime \prime}}}{\frac{\Delta Q_{W}^{\prime \prime}}{T_{W}^{\prime \prime}}}=\frac{H\left(Y^{\prime \prime} \mid X^{\prime \prime}\right)}{H\left(Y^{\prime \prime}\right)}=\frac{T_{0}}{T_{W}^{\prime \prime}}, T_{W}^{\prime \prime}=T^{*}, \text { cyklus } \mathcal{O}^{\prime \prime} \\
\beta=\frac{\Delta Q_{0}}{\Delta Q_{W}}=\frac{\frac{\Delta Q_{0}}{T_{W}}}{\frac{\Delta Q_{W}}{T_{W}}}=\frac{H(X \mid Y)}{H(X)}=\frac{T_{0}}{T_{W}}, \text { cyklus } \mathcal{O} \\
\frac{\beta}{\beta^{\prime \prime}}=\frac{T_{W}^{\prime \prime}}{T_{W}}=\frac{T_{0}^{*}}{T_{W}} \triangleq \beta^{*}
\end{gathered}
$$


and then, by (30) and (31) is writable that

$$
\frac{\Delta A^{*}}{\mathrm{k} T_{W}}=H(X) \cdot\left(1-\beta^{*}\right)=H(X) \cdot\left[1-\frac{H(X \mid Y) \cdot H\left(Y^{\prime \prime}\right)}{H\left(Y^{\prime \prime} \mid X^{\prime \prime}\right) \cdot H(X)}\right]>0
$$

It is ensured by the propositions $T_{W}>T_{W}, T_{0}=T_{0}$ and also by that fact that the loss entropy $H(X \mid Y)$ is described and given by the heat $\Delta Q_{0}=\Delta Q^{\prime \prime}{ }_{0}$. But in our combined cycle $\mathcal{O O}^{\prime \prime}$, it is valid too that

$$
H(X)=\frac{\Delta Q_{W}}{\mathrm{kT}_{\mathrm{W}}}=\frac{\Delta Q_{W}^{\prime \prime}}{\mathrm{kT}_{\mathrm{W}}^{\prime \prime}}=H\left(Y^{\prime \prime}\right) \quad\left[=\frac{\Delta Q_{W}^{\prime \prime}}{\mathrm{kT}_{0}^{*}}\right]
$$

and we have

$$
\frac{H(X \mid Y)}{H\left(Y^{\prime \prime} \mid X^{\prime \prime}\right)}=\beta^{*}<1
$$

For the whole information entropy $\frac{\Delta A^{*}}{\mathrm{k} T_{W}}$ (the whole thermodynamic entropy $\mathcal{S}_{\mathcal{C}}$ in information units) and by following the previous relations also it is valid that

$$
\begin{aligned}
\frac{\Delta A^{*}}{\mathrm{k} T_{W}} & =H\left(Y^{\prime \prime}\right)-H\left(Y^{\prime \prime}\right) \cdot \beta^{*}=H\left(Y^{\prime \prime}\right) \cdot\left(1-\frac{T_{0}^{*}}{T_{W}}\right) \\
& =H\left(Y^{\prime \prime}\right) \cdot\left[1-\frac{H(X \mid Y)}{H\left(X^{\prime \prime} \mid Y^{\prime \prime}\right)}\right]
\end{aligned}
$$

And thus, the structure of the information transfer channel $\mathcal{K}$ [expressed by the quantity $H(X \mid Y)]$ is measurable by the value $H^{*}\left(Y^{*}\right)$ from (32) and (35). Symbolically, we can write, using a certain growing function $f$,

$$
H^{*}\left(Y^{*}\right)=\frac{\Delta A^{*}}{\mathrm{k} T_{W}} \cong f[H(X \mid Y)]>0
$$

The cycles $\mathcal{O}, \mathcal{O}^{\prime \prime}$, and $\mathcal{O O}^{\prime \prime}$ are the Carnot Cycles, and thus from their definition and construction, they are imaginatively ${ }^{16}$ in principle, the infinite cycles; in each of them the following criterion of an infinite cycle (see [12]) it is valid inevitably,

$$
T\left(X^{[\cdot]} ; Y^{[\cdot]}\right)=H\left(X^{[\cdot]}\right)-H\left(X^{[\cdot]} \mid Y^{[\cdot]}\right)=H\left(Y^{[\cdot]}\right)>0 \text { and } \Delta \mathcal{S}_{\mathcal{L}}^{[\cdot]}=0
$$

The construction of the cycle $\mathcal{O} \mathcal{O}^{\prime \prime}$ enables us to recognize that the infinite cycle $\mathcal{O}$ is running. In our case, it is the infinite cycle from (5), (6) and also from $[4,8,10]$,

$$
\begin{array}{llll}
Q(\mathbb{X}, Y), & Q[\mathbb{X}, \Phi[Q(\mathbb{X}, Y)]], & Q[\mathbb{X}, \Phi[Q(\mathbb{X}, \Phi[Q(\mathbb{X}, Y)])]], \ldots \\
Q\left(\mathbb{N}_{0}, Y\right), & Q\left[\mathbb{N}_{0}, \Phi\left[Q\left(\mathbb{N}_{0}, Y\right)\right]\right], & Q\left[\mathbb{N}_{0}, \Phi\left[Q\left(\mathbb{N}_{0}, \quad \Phi\left[Q\left(\mathbb{N}_{0}, Y\right)\right]\right)\right]\right], & \ldots
\end{array}
$$

${ }^{16}$ When an infinite reserve of energy would exist. 


\section{Author details}

\section{Bohdan Hejna}

Department of Mathematics, University of Chemistry and Technology, Prague, Czech Republic

*Address all correspondence to: hejnab@vscht.cz

\section{IntechOpen}

(C) 2019 The Author(s). Licensee IntechOpen. This chapter is distributed under the terms of the Creative Commons Attribution License (http://creativecommons.org/licenses/ by/3.0), which permits unrestricted use, distribution, and reproduction in any medium, provided the original work is properly cited. (c) BY 


\section{References}

[1] Hejna B. Recognizing the infinite cycle: A way of looking at the halting problem. In: Dubois DM, editor. Proceedings of the Tenth International Conference CASYS'11 on Computing Anticipatory Systems, Lecture on CASYS'11 Conference, 8-13 August 2011. CHAOS; 2012. ISSN: 1373-5411

[2] Hejna B. Informační termodynamika III.: Automaty, termodynamika, přenos informace, výpočet a problém, zastavení. Praha, VŠCHT Praha; 2013. ISBN: 978-80-7080-851-1

[3] Hejna B. Information thermodynamics and halting problem. In: Bandpy MG, editor. Recent Advances in Thermo and Fluid Dynamics. Croatia, Rijeka: InTech; 2015. pp. 127-172. ISBN: 978-953-51-2239-5. Available from:http://www.intechopen. com/books/recent-advances-in-thermoand-fluid-dynamics

[4] Hejna B. Gödel proof, information transfer and thermodynamics. In: Lecture on IIAS Conference; 3-8 August 2015; Baden-Baden, Germany; Journal IIAS-Transactions on Systems Research and Cybernetics. The Inernational Institute for Advanced Studies in System Research and Cybernetics; 2015; 15(2). IBSN: 978-897546-13-0

[5] Gödel K. Über formal unentscheidebare Satze der Principia Mathematica und verwandter Systeme I. von Kurt Godel in Wien; Monatshefte fur Mathematik und Physik 1931;38:173-198

[6] Gödel K. On Formally Undecidable Proposition of Principia Mathematica and Related Systems. Vienna; 1931 (translated by B. Metzer)

[7] Včelař F, Frýdek J, Zelinka I. Godel 1931. Praha: Nakladetelství BEN; 2009
[8] Hejna B. Information transfer and thermodynamics point of view on Gödel proof. In: Thomas C, editor. Ontology in Information Science; College of Engineering Trivandrum, India. InTech; 2017/18. ISBN: 978-953-51-5354-2, ISBN: 978-953-513888-4. Print ISBN: 978-95351-3888-4. Available from: http://www. intechopen.com/books/ontology-in-inf ormation-seience; OAI link: http:// www.intechopen.com/oai/?verb= ListIdentifiersmetadataPrefix $=$ oai $_{d}$ cset $=$ 978-953-51-3887-7; Scientometrics on: https://www.intechopen.com/books/ statistics/ontology-in-informationscience/information-transfer-andthermodynamic-point-of-view-ongödel-proof; Indexing: WorldCat, Base, A to Z, IET Inspect, Scirus, Google Scholar

[9] Hejna B. Informační termodynamika IV.: Gödelovy věty, přenos informace, termodynamika a Caratheodoryho věty. Praha: VŠCHT Praha; 2017. ISBN: 978-80-7080-985-3

[10] Hejna B. Gödel and Caratheodory theorems. In: Lecture on IIAS Conference; August 2017; Baden-Baden, Germany. Journal IIAS-Transactions on Systems Research and Cybernetics.

2017;1. ISSN:1609-8625. ISBN:

978-1897456-42-0

[11] Hála E. Úvod do chemické termodynamiky. Praha: Academia; 1975

[12] Hejna B. Information thermodynamics. In: MorenoPiraján JC, editor. ThermodynamicsPhysical Chemistry of Aqueous Systems. Croatia, Rijeka: InTech; 2011. pp. 73-104. ISBN: 978-953-307-979-0. Available from: http://www.intechopen. com/articles/show/title/information-the rmodynamics 


\title{
Knowledge Patterns within the Conception of Semantic Web
}

\author{
Martin Žáček, Alena Lukasová, Marek Vajgl and Petr Raunigr
}

\begin{abstract}
The article tries to contribute to answer a question if the general concept of knowledge pattern with its sub-concepts covers a great majority of the approaches used under this term in computer science literature. At one case, specialized software design patterns in the frame of object-oriented methodology become a very well used tool for software praxis; at a different case, there exists a large packet of tools for creating ontologies of various areas. As a third case, also RDF-based networks of linked data could be seen as knowledge patterns characterizing at least structures or defined activities of some social, working, or other organizations. We propose here to see the problem of knowledge pattern from knowledge representation especially at directions where the goal of using knowledge pattern meets the general goal of the semantic web. The motivation of this article is to apply knowledge patterns in the semantic web because knowledge at a higher professional level can and should usually be given in such a way that their specialized formal expertise incorporates the key to understanding their meaning.
\end{abstract}

Keywords: pattern, knowledge, RDF, CFL, semantic web

\section{Introduction}

Generally, the concept of knowledge pattern [1] modeling appears in knowledge engineering, apparently due to the corresponding concept of a design pattern in software development in the frame of object-oriented methodology. But the difference is mainly in the area of the two points of view. While the design pattern is focused toward general principles of software creation in terms of practices, structure, or behavior properties, the corresponding specifications of knowledge patterns need to take into focus minimally the concept of knowledge, its properties, and cases. While in the case of declarative knowledge it simply involves acquiring new knowledge or its new application from a given knowledge base, all within the first-order logics formalism, for procedural knowledge a generally acceptable formal language and approach until now has not been found. But knowledge pattern for procedural knowledge case gives us a possibility to use similar rules of design patterns as well as in the case of software development. If moreover knowledge pattern has been embedded into the semantic web concept [2] environment with a seeing the world throw the RDF principle [3], it represents a new quality in the sense that content and form become easy-to-use for computers and comprehensible to users without deeper penetration into the principles of knowledge engineering. 


\section{How to take the topic of knowledge into the AI?}

At the Cambridge English Dictionary, we can read a definition of the meaning of the concept of knowledge as follows:

An understanding of or information about a subject that you get by experience or study, either known by one person or by people generally.

The question in the title of the paragraph with a corresponding Cambridge explanation seems to be the basic one. But our goal must be a bit more different from a topic at philosophy (gnoseology); we only try to generalize a bit an orientation about the concept of knowledge pattern within a formal representation language used in AI.

\subsection{What is knowledge in praxis about?}

In the epistemological area of artificial intelligence, we encounter formal manipulation of knowledge. Knowledge is based on information, and this information is based on data [4].

To be able to work with knowledge in a form suitable for computer implementation, it is necessary to introduce some formalism-a representative language. This language must be able to reflect the relationship between knowledge of the world, stored in human minds, and knowledge written in formal means.

Knowledge is information that is usable and divisible, respectively, in relation to other information [4].

In other words:

- In one case, it is about what entities a specified (reference) "world" consists of and what are their properties and relationships. The description of the status of participating entities has a declarative character in this case.

- In the latter case, it is about the starting state of the "world" and using rules for reaching a target state. The description here is of a procedural form, capturing the crucial interstates through which the process passes.

\subsection{Knowledge elements and knowledge components}

Knowledge elements are bounded to a certain knowledge base written usually in a special formal approach (or language syntax). The elements are atoms that cannot be further divided. An important feature of atoms is their independence from external contexts. This is especially important for their applications and reuse. Knowledge must be lasting about the knowledge base in which knowledge can be manipulated; it must have a permanent meaning.

Knowing elements linked to a given knowledge base can be composed into knowledge components, whose syntax and meaning is created through the grammatical rules and (logical) composition of participating atoms [5].

Knowledge components are conceptually dependent on the knowledge model used and on its required properties. The factors that make up the overall character of the knowledge component in the composition should be the following:

- The functionality is reflecting and sharing a specified relationship between the start state and target state. 
- Reliability, which means that the component is mature.

- Applicability and portability are that the component is understandable and appropriately applicable to allow wider use.

- Modifiability, i.e., the ease of partial changes in the stability of the basic properties.

The meaning of the elementary or compound knowledge is secured at all points above if the RDF model has been chosen to represent a conceptual reality.

\subsection{What is a knowledge pattern?}

The term "knowledge pattern" was first used in $[1,6]$. While building ontologies or knowledge bases, one can see that some structures of modeled knowledge are the same. These same structures of knowledge can be captured as knowledge patterns. Knowledge patterns are general structures (patterns) of knowledge, which are not a part of the target knowledge base. They can be included into a target knowledge base by renaming their nonlogical symbols. This renaming is called morphism. The morphism is an important part of using knowledge patterns $[6,7]$.

Presently, there is no direction for capturing knowledge patterns. We propose to model knowledge patterns in RDF graph models $[8,9]$ of the semantic web.

Going through the topics of knowledge pattern at the web, we have to meet the following more or less similar approaches:

- Seeking general knowledge pattern as (1) a small ontology [10] within a well-specified part of the (reference) world.

- Seeking general knowledge pattern as (2) a frame structure of linked data of some oft appearing kernels of together-linked facts about everyday life, as is the case of a firm leading structure and tasks of participants.

- Seeking general knowledge as (3) the helping means on how to construct a special product or software of expected properties.

The first and second cases represent more or less declarative approaches to reach the main result from them. They are of hierarchical strictures of classes with their subclasses. The third one represents a procedural knowledge, but it is difficult to find a common principle of building results as a formal description. But in this case, there is a rich database of very useful prescribes for a big scale in praxis.

A specification of the concept in the title within the formal representation approach can be something like the following:

A knowledge pattern [6] concerns "holding as true of a set of sentences or rules about a specified piece of the world-either known by one person or by people generally, all expressed by formal means."

\subsection{Knowledge pattern (KP) within a knowledge representation}

In general, in the field of types of knowledge, two basic cases corresponding to the kinds of knowledge at Section 2.1 of the access in formalization should be considered: 


\section{Declarative knowledge representation}

\section{Procedural knowledge representation}

In summary, a common feature in both types of pattern specification is their generic validity within a given environment, verification by historical development, and long-term experience in their applications. If moreover knowledge design patterns are embedded in the semantic web concept environment, they represent a new quality in the sense that their content and form become comprehensible to users without deeper penetration into the principles of knowledge engineering.

In terms of global formal representation, the question is how to create:

3. A unifying approach is generally applicable to the representation of knowledge (sometimes also appearing under the working name "framework approach") based on both of the above approaches, enriched by those means of representation that are missing in the first or second access.

We would like to present here a proposal to use instead of the ontology design patterns [11] for the approach to the topic a simple knowledge pattern bounded especially to specialized kind of knowledge.

\subsection{Knowledge pattern (KP) versus special design patterns}

A majority of authors use the term ontology design pattern (OP) because the OP is, in fact, a modeling solution of solving a recurrent ontology design problem. We would like to show here the fact that the attribute "ontology" is not a necessity in the case of using RDF modeling principle that carries this property as an implicit one.

Definition of the OP according to the authors of the article [11]:

Ontology design pattern (or only OP) is a modeling solution to solve a recurrent ontology design problem.

Authors [12] have identified within web documents several types of OPs and have grouped them into six families:

- Structural OPs

- Correspondence OPs

- Content OPs (CPs)

- Reasoning OPs

\section{- Presentation OPs}

- Lexico-syntactic OPs

Knowledge patterns [13] of declarative or procedural knowledge cases give a possibility how to use rules of similar design patterns as well as in the case of software development. In both cases a natural language plays an important role, and definition in a special language is not necessary.

While examples of software design pattern applications can be found to a large extent, knowledge patterns tend to be related only in a few types of problem areas and their language representations. 


\section{Knowledge in formal representation}

\subsection{Representation of declarative knowledge}

At the beginning, the principle of seeing a simplified world was considered an abstraction of the real world to be modeled. Usually, the E-R principal is to be chosen.

The process of conceptualization has stabilized during the development of the means of modeling reality onto the well-established world view as a set of entities with certain characteristics and mutual relationships.

Now at first a generally recognized and application-proven way of using a formal language is given for the sake of building a formal description of concepts and their properties based on the basic (conceptual) level of the E-R model world abstraction [14]. Conceptualization is partially subordinated to the expected formal language syntax. The semantics of the language of knowledge represented in this way of seeing the world ought to be derived from declarative descriptions of the properties and relationships of the entities of the given reference world. They are then formally represented according to the rules applicable in the reference world. From anchoring corresponding concepts on the web or the semantic web, the current state of development has to choose the use of the principle of RDF modeling [15]. Using the RDF [15] data model representation gives a possibility of graphic representation of RDF triples [16] as vectors expressing corresponding knowledge elements (see Figure 1): < subject $><$ property $><$ object $>$.

RDF describes the resource (as a subject), which has some property with a corresponding value (object). The RDF model is based on associative (semantic) networks $[17,18]$.

In the following figure (Figure 1) we can see the conversion of the sentence "Marek teaches the subject of pgm languages." Clearly here we see that the subject is "Marek," the object is "pgm language," and the property is "teaches."

\subsection{Representation of procedural knowledge}

The most successfully applied approach to the representation of procedural properties of the modeled world is the output of the process of algorithmic representation of the modeled reality, which builds a formal description of the reference world based on the graphic expression of the formalization-a flowchart of the basic elements of human activity in it.

Just as a formalization of declarative knowledge is guided by a conceptual flowchart, procedural knowledge [19] is the guiding factor of the problem of its algorithm, i.e., the way of seeing the process described based on elementary programming language components with special language syntax. The

RDF tripple

<subject> <property> <object>

marek teaches pgm languages
RDF vector

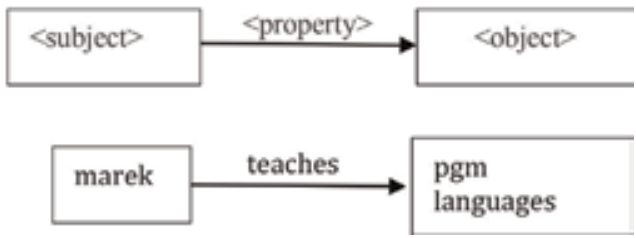

Figure 1.

Representation of the fact "Marek teaches the subject of pgm languages" using a RDF triple and with the help of $R D F$ graph vectors. 
representative language used to simulate the performance of the modeled activity usually has a specific form of a programming language reflecting the characteristics of the modeled activity and the practical application needs.

Algorithms typically ignore entities with their properties and relationships, with the interconnection of modeling activities in the process providing "data," usually without any closer anchoring in entity representations. The problem solves the following frame approach of modeling.

\subsection{The framework character of knowledge}

Algorithmic representations of the modeled world with its procedural properties, as well as in the case of point 1 , must take into a focus relation to elementary entities and their properties and relationships of a modeled world. It should, therefore, use a representation method based on the RDF model corresponding to point 1 , with the terminology relating to entities with their properties and relationships being anchored in the chosen dictionary (ontology) to which access created by the RDF model [20] has been bound.

E.g. the language UML (as a means of describing RDF-modeled reality) would allow UML diagrams to data retrieve into the represented process and their belonging to home entities within the chosen ontology.

\section{Knowledge pattern in a semantic web context}

\subsection{RDF modeling principle and knowledge patterns}

Creating data for the semantic web means conceptualizing world using E-R model with a participation of a key ontology because of sharing and reusing formalized knowledge representation. Each data item can take its meaning from a standardized description of web resources within the proper ontology using its URI identifier.

\subsection{Semantic web patterns and anti-patterns}

The RDF as a general framework for describing, replacing, and reusing metadata represents the technological foundation of the semantic web. From anchoring corresponding concepts on the web (or the semantic web), the current state of development is the use of the RDF $[4,21]$ modeling principle.

RDF describes the resource, which has some properties, and these properties have corresponding values (Figure 1). While the subject defines the source, the property determines its nature and at the same time expresses the relationship between the subject and the object.

The semantic web idea is based on the RDF technology [22], which integrates the web language syntax and the naming of its elements by URIs. So a content presented on the semantic web has a well-defined meaning and allows a better understanding of both people and software agents.

The semantic web provides a common framework that allows data to be shared and reused.

It also emphasizes the ease of understanding and applicability of documents on the web, especially easy usability of knowledge model as well as knowledge pattern approach. 


\subsubsection{Definition}

The knowledge pattern is a general type of component knowledge of proven success, often with a design concept of good practice, a process of structuring, to create the architecture of component knowledge. It may be declarative, procedural, or frame-like.

An anti-pattern is a common often accompanying process phenomenon that is not involved in solving the problem (wrong solutions or "worst-case" solutions). Unlike the model, the anti-model generally describes individual non-model cases and highlights a general solution to recurring problems.

In its formal representation, the knowledge pattern should:

- Be anchored within a specific knowledge base with a given semantics.

- If possible be used for the formal representation of knowledge through a language with easy-to-understand interpretations (preferably graphical).

- Have individual atomic components, of which the pattern/anti-pattern is composed, clearly defined, and described.

\subsection{An example of a knowledge pattern for the case of declarative knowledge}

As an example of the knowledge pattern, we shall show in this paragraph the case of a declarative knowledge at its basic logical form.

In the field of formal logic [23], the declarative knowledge design pattern extends deep into the history of formal systems. At a time when philosophers and mathematicians changed their orientation from specific individual descriptions to general principles of reasoning, they came as a result of general principles of deduction in formal logics [23]. Procedures as a rule modus ponens or the resolution inference rule in propositional or predicate logic represent in terms of the ongoing development of artificial intelligence typically general guidance on how to derive from the assumption's logical consequences, respectively how to arrive at a logical deduction on the arguments that confirm or reject given assertions. This is nothing more than a guideline on the application of a knowledge pattern.

\section{Rules of deduction in formal logics in the role of declarative knowledge patterns}

The publication on formal logic and semantic web [4] lists several examples of the application of a resolution deduction rule as a knowledge model allowing the derivation of a logical consequence from given assumptions.

The following example illustrates the use of RDF CFL resolution derivation rule that obtained a logical consequent from a knowledge base [4].

An example of immigration rules for Europe is given as a knowledge base in the language of the first-order predicate logic, which, as well known, is not one of the easy-to-understand and usable languages of formal logic. However, there is a way of transferring (according to well-known rules) to the special clause of CFL [24], which has been before based on conceptualization according to the RDF principle. To express its concepts and their properties and relationships, this language, on behalf of RDF CFL [25], uses exclusively the binary predicates. Consequently, a corresponding graphical representation has been used, playing an important role in the semantic web. 


\subsection{Example}

Immigration rules for citizen as a knowledge base in the FOPL

1. $\forall \mathrm{x} \forall \mathrm{y}(\operatorname{stateEU}(\mathrm{y}) \& \operatorname{citizen}(\mathrm{x}, \mathrm{y}) \rightarrow \operatorname{enter}(\mathrm{x}))$

2. $\forall \mathrm{x} \forall \mathrm{y}(\neg \operatorname{stateEU}(\mathrm{y}) \& \operatorname{citizen}(\mathrm{x}, \mathrm{y}) \&$ has_visa $(\mathrm{x}) \rightarrow$ enter $(\mathrm{x}))$

citizen(anne, aus), citizen(achim,tur), has-visa(achim), stateEU(aus), ᄀstateEU(tur).

\subsubsection{Resolution rule in a generalized form}

Creating a CFL clause for using the resolution rule of the RDF CLF language according to the scheme:

$$
\frac{<\text { CFL clause } 1>\quad<\text { CFL clause } 2>}{<\text { the logical consequent of } 1 \text { and } 2>}
$$

As the set of the RDF CFL clausal form contains only positive atoms, we prepare for the resolution rule those basic atoms that express positive statements about the participating persons and states in our example (Table 1):

1. stateEU(aus), citizen(anne, aus) $\rightarrow$ enter(anne).

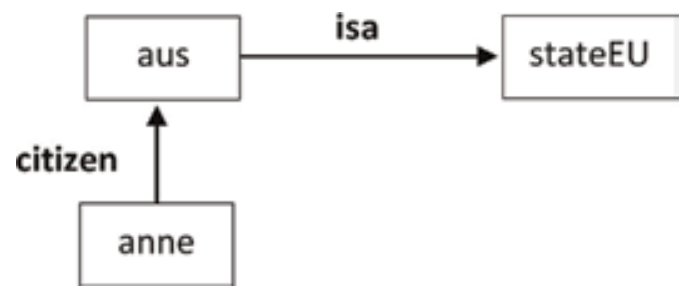

Verbal expression: "Anne is a citizen of Austria, which is a state of EU."

2. RDF CFL instance of the (1.)

$$
\rightarrow \text { stateEU(aus). }
$$

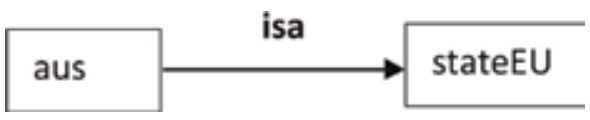

Verbal expression: "Austria is a state of EU." citizen(anne, aus) $\rightarrow$ enter(anne).

Logical consequent after a using of the resolution rule.

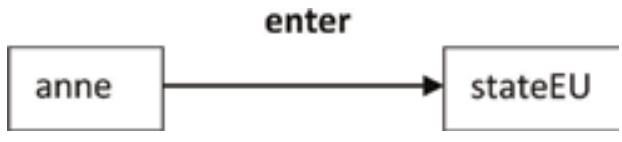

Verbal expression: "Anne can enter to any state of EU." Similar examples can be seen in the publication [26]. 


\begin{tabular}{ll}
\hline Entity & IRI (URI) \\
\hline state & http://dbpedia.org/page/State_(polity) \\
\hline citizen & http://dbpedia.org/page/Citizenship \\
\hline aus & https://www.wikidata.org/wiki/Q40 \\
\hline anne & http://dbpedia.org/page/Anne \\
\hline visa & http://dbpedia.org/page/Travel_visa \\
\hline enter & https://www.wikidata.org/wiki/Q161935 \\
\hline isa & is a \\
\hline
\end{tabular}

Table 1.

IRI of the example (RDF).

\section{A proposed structure of records in the knowledge pattern archive}

Design Name

Objective

Also known as

Context

Motivation $^{\star}$

Usage

Structure

Participants

Consequences

Known uses

* contradictory forces.
A unique name that adequately describes the knowledge pattern and reference it helps identify.

A description of the goal for which the knowledge pattern originated.

Other names for the same knowledge pattern.

A situation where a description of the knowledge pattern is useful.

Description of the problem to be solved by the given knowledge model.

Situations in which the pattern can be used. This is the context of a knowledge model.

Graphic representation of the knowledge pattern.

List of classes and objects that use this knowledge pattern and their role in the design.

A description of the results, side effects, and problems that the pattern makes use of.

Examples of practical use of the pattern.

\section{The RDF CFL graph resolution rule in the proposed pattern archive}

Design Name

Objective

Also known as
Context

Motivation ${ }^{\star}$

Usage

Structure
RDF CFL graph resolution rule.

The pattern in the form of a rule serves a possibility of obtaining further knowledge like consequents of a knowledge base.

Some of the consequents hidden before become visible and could make the whole content more understandable.

Better level of usability of a knowledge base. as an example: solving the consequent from two clauses in the graph representation.

Symbolic expression of a deduction.

$<$ graph of clause $1>$ < graph of clause 2>

$<$ graph of the logical consequent $>$ 
Participants

Consequences

Known uses

${ }^{\star}$ contradictory forces.
Graph records of two clauses with at least one identical atom.

If an empty clause has been obtained, it means an unsolvability of the input clauses without any consequent.

An example of practical use of the pattern seen above at example 1.

\section{Conclusion}

The idea of a semantic web can be a handy hand given to those who still feel at least a mistrust, if not resistance, to take knowledge from websites. Knowledge from areas at a high level of science can be discouraged by a layman, among other things, due to ignorance of the special language of the field in question. Thus, any known common patterns, which can be called "knowledge patterns," are not recognized in the new context, so they are generally not considered to be reusable. It is, therefore, necessary to look at approaches that can convey wider usability. The semantic web has this approach in the very description of its definition. Knowledge at a higher professional level can and should usually be given in such a way that their specialized formal expertise incorporates the key to understanding their meaning. Our goal is not to create a universal tutorial, but we must integrate the semantics of knowledge presented in the formal language direct into its syntax. The above graphic example is illustrative.

\section{Author details}

Martin Žáček*, Alena Lukasová, Marek Vajgl and Petr Raunigr

Faculty of Science, University of Ostrava, Ostrava, Czech Republic

*Address all correspondence to: martin.zacek@osu.cz

\section{IntechOpen}

(C) 2019 The Author(s). Licensee IntechOpen. This chapter is distributed under the terms of the Creative Commons Attribution License (http://creativecommons.org/licenses/ by/3.0), which permits unrestricted use, distribution, and reproduction in any medium, provided the original work is properly cited. (c) BY 


\section{References}

[1] Clark P, Thompson J, Porter B. Knowledge patterns. In: Staab S, Studer R, editors. Handbook on Ontologies. Berlin: Springer-Verlag; 2004. pp. 191-207. ISBN: 3-540-40834-7

[2] Berners-Lee T, Hendler J, Lassila O. The semantic web. Scientific American. 2001;284(5):28-37

[3] Swartz A. The Semantic Web in Breadth [Internet]. 2002. Available from: http://logicerror.com/semantic Web-long

[4] Lukasová A, Žáček M, Vajgl M, Telnarová Z. Formal Logics and Semantic Web (Formální Logika a Sémantický Web). Plzeň: ZČU Plzeň; 2015. ISBN: 978-80-261-0408-7

[5] Schwitter R. Controlled natural languages for knowledge representation. In: Proceedings of the 23rd International Conference on Computational Linguistics; 2010. pp. 1113-1121

[6] Miarka R, Žáček M. Knowledge patterns for conversion of sentences in natural language into RDF graph language. In: Proceedings of the Federated Conference on Computer Science and Information Systems. USA: IEEE Computer Society Press; 2011. pp. 63-68. ISBN: 978-1-4577-0041-5

[7] Miarka R, Žáček M. Knowledge patterns in RDF graph language for English sentences. In: Proceedings of the Federated Conference on Computer Science and Information Systems. USA: IEEE; 2012. pp. 109-115. ISBN: 978-8360810-48-4

[8] W3C. Resource Description Framework (RDF): Concepts and Abstract Syntax [Internet]. Available from: http://www.w3.org/TR/ 2004/REC-rdf-concepts-20040210/
[9] W3C. RDF Primer [Internet]. Available from: http://www.w3.org/TR/ 2004/REC-rdf-primer-20040210/

[10] Žáček M. Ontology or formal ontology. In: International Conference of Numerical Analysis and Applied Mathematics 2016 (ICNAAM 2016): AIP Conference Proceedings. American Institute of Physics Inc.; 2017. ISBN: 978-073541538-6

[11] Gangemi A. Ontology design patterns for semantic web content. In: Gil Y, et al. editors. ISWC 2005; 2005. pp. 262-276

[12] Rech J, Feldmann R, Fraunhofe E. Knowledge Patterns [Internet]. Available from: http://joerg-rech.com/ Paper/RechFeldmannRas_EncycKM_ KnowledgePatterns.pdf

[13] Rech J, Decker B, Jedlitschka A. The quality of knowledge: Knowledge patterns and knowledge refactorings source. International Journal of Knowledge Management (IJKM). 2007. pp. 578-586

[14] Žáček M, Lukasová A, Vajgl M. Ontology languages for semantic web from a bit higher level of generality. In: 10th International Scientific Conferences on Research and Applications in the Field of Intelligent Information and Database Systems; ACIIDS 2018: Lecture Notes in Computer Science 2018-03-19, Dong Hoi City, Vietnam. Switzerland: Springer Verlag; 2018. pp. 275-284. ISBN: 978-331975419-2

[15] W3C. Resource Description Framework (RDF) Model and Syntax Specification [Internet]. 2008. Available from: http://www.w3.org/TR/ 1999/REC-rdf-syntax-19990222

[16] Lukasová A, Žáček M, Vajgl M. Reasoning in formal systems of 
extended RDF networks. In: 9th Asian Conference on Intelligent Information and Database Systems (ACIIDS): Intelligent Information and Database Systems, Kanazawa, Japan. Switzerland: Springer Verlag; 2017. pp. 371-381. ISBN: 978-3-319-54430-4

[17] Findler NV, editor. Associative Networks: Representation and Use of Knowledge by Computers. New York: Academic Press; 2004

[18] Lukasová A, Vajgl M. Žáček M. Knowledge represented using RDF semantic network in the concept of semantic web. In: Proceedings of the International Conference on Numerical Analysis and Applied Mathematics 2015 (ICNAAM-2015). USA: American Institute of Physics Inc.; 2016. ISBN: 978-0-7354-1392-4

[19] Vajgl M, Lukasová A. Žáček M. Knowledge bases built on web languages from the point of view of predicate logics. In: Proceedings of the International Conference on Applied Mathematics and Computer Science, ICAMCS 2017: AIP Conference.

American Institute of Physics Inc.; 2017. ISBN: 978-073541506-5

[20] Lukasová A, Žáček M. Creating words by inflexion and derivation in RDFCFL graphs. WSEAS Transactions on Computer Research. 2016;4(23): 208-212. ISSN: 1991-8755

[21] Staab S, Erdmann M, Maedche A, Decker S. An extensible approach for modeling ontologies in RDF (S). In: Knowledge Media in Healthcare: Opportunities and Challenges. IGI Global; 2002. pp. 234-253. https://www. igi-global.com/chapter/knowledge-med ia-healthcare/25416

[22] Gangemi A. Ontology design patterns for semantic web content. In: International Semantic Web Conference. Berlin, Heidelberg: Springer; 2005. pp. 262-276
[23] Baader F, Horrocks I, Sattler U.

Description logics as ontology languages for the semantic web. In: Mechanizing Mathematical Reasoning. Berlin, Heidelberg: Springer; 2005. pp. 228-248

[24] Richards T. Clausal Form Logic. An Introduction to the Logic of Computer Reasoning. Boston, MA, USA: AddisonWesley Longman Publishing Co., Inc.; 1989

[25] Lukasová A, Žáček M, Vajgl M. Carstairs-McCarthy's morphological rules of english language in RDFCFL graphs. In: International Conference on Applied Physics, System Science and Computers (APSAC). Springer Lnee; 2016. pp. 169-174. DOI: $10.1007 / 978-3-$ 319-53934-8_20

[26] Žáček M. Ferdiánová V. Solving logic problems with associative networks in the course of knowledge representation. In: International Conference on Industrial Technology and Management Science: Advances in Computer Science Research 2014 China. France: Atlantis Press; 2015. pp. 121-124. ISBN: 978-94-6252-123-0 


\title{
Ontology Language XOL Used for Cross-Application Communication
}

Jinta Weng, Jing Qiu and Ying Gao

\begin{abstract}
The 2000s may be the flourishing time of the topic of ontology. Specialists and scholars concentrated to define ontology effectively and formulated uniform ontology protocol. Ontology language can be classified into SHOE, OML, XOL, OIL, OWL, and RDFs by different protocols and syntaxes. As for effective exchange of the different ontology messages in different applications, US bioinformatic community and researcher develop a XML-based ontology language. With the simplified OKBC-Lite protocol and flexible XML syntax, XOL offers the ways to define an ontology with the human-readable XML, simplified protocol, and compatible interface. In this chapter, we will introduce its motivation from history, orientation in development, semantic usage, and interpreted example in detail.
\end{abstract}

Keywords: ontology exchange language, Ontolingua, XOL, open knowledge base, Semantic Web

\section{Introduction}

Internet had maintained a rapid development between the 1990s and 2000s, which not only gives birth to various applications, abundant network facilities, and diverse websites but also accelerates the next generation of Semantic Web. After Berners-Lee put forward the imaginary structure of Semantic Web in 1998, W3C with many semantic work teams is dedicated to develop the technical standard of Resource Description Framework [1]. As ontology is the essence and basic of a resource, technical combinations of paradigm and languages are used to define it.

\subsection{Background}

Knowledge engineering has become an essential part of expert system in artificial intelligence. It is important to define the specific knowledge, also known as domain database or knowledge base, for multiple applications. However, traditional knowledge base just reveals the key and value of the data, thus paying less attention on ontology.

Ontology is the description and formulization of thing. By full-semantic and expressive ontology, more information and relationship are able to excavate. In order to build more humanistic and intelligent system, scholars had developed different ontology languages. Although many ontology languages give methods to solve the ontology definition. However, a new language or ontology protocol should also be formulated to deal with the cross-application problem. 


\subsection{Motivation}

Accompanying with the development of Internet, more infrastructure, application, and knowledge base are generated. In normal knowledge supported systems, domain expert will first considerate the software environment and self-knowledge background and then choose the suitable knowledge scheme and ontology for the system. However, when it comes to the cross applications or large knowledgeassisted system, ontologies in system need to be reused. First, knowledge scheme in different systems may exist difference from the expert's personal cognize.

Second, it offers several ontology languages for each system; thus, different ontology schemes can show in different formats, which make it hard to communicate in different applications. Third, the increasing demand of openness and the sharing lead of ontology could be exchange. Therefore, an ontology exchangeable protocol or new ontology language supporting to exchange should be redefined.

To realize the need of an evaluation on ontology in bioinformatics, several researchers on the US evaluation team developed a new specific ontology language-XOL [2]. By flexible XML expression and simplified protocol, XOL (xml-based ontology exchange language) is able to express and exchange different ontology information across incompatible applications.

\subsection{Definition}

$\mathrm{XOL}$ is an ontology language developing for exchange ontology in cross applications. It takes inspiration from OKBC (a protocol used for open knowledge base, see in Ref. [3]) and Ontolingua (another ontology used for reusing and editing ontology, see in Ref. [4]). Its syntax is based on human-readable and high compatible XML document. XOL can also respect as one effective intermedia language in ontologies' use, exchange, negotiation, and cocreation.

\subsection{A simple example}

Note the following XOL definitions:

$<$ class $>$

$<$ name $>$ [class-name] $</$ name $>$

$</$ class $>$

$<$ slot $>$

$<$ [slot-attribute] $></[$ slot-attribute $]>$

$<$ slot $>$

$<$ individual $>$

$<$ name $><$ /name $>$

$<$ type $><$ /type $>$

$<\ldots></ \ldots>$

$<$ individual $>$

All of above XOL elements are pertained to all ontologies. Between the pair of $<$ class $><$ /class $>$ defines the basic information of this ontology, like the name of the class during the tag pair of $<$ name $><$ /name $>$.

Pair of $<$ slot $></$ slot $>$ will depict the attribute and restriction of the class, like value's type of the attribute and the data restriction.

The last tag <individual> </individual > will give an instance of self-class or multiclass. It is not allowed to use the subclass as the individual element.

With the human-readable and self-defined XML syntax, XOL can express the ontology in a concise way. However, it may also lead to the ontology inconformity 
while using XML syntax merely or personally. A more restrictive and stationary tag OKBC-Lite was chosen soon.

\section{Why is XOL based on XML?}

Generally speaking, each ontology language makes up for using syntax and language protocol. To realize the essence of XOL, we will show the different classifications of ontology languages based on the syntax and semantic rules.

According to the use of syntax, we can classify the ontology languages into three types as follows:

HTML format: Hypertext Markup Language (HTML) is the basic document mark of the current web. To extend the semantic character of the HTML, ontology language like SHOE offers an effective way to support semantic annotation by more extended webpage label.

XML format: Extensive Markup Language (XML) is a more human-readable and concise document for storing and defining different data. Ontology document made by XML format can easily locate by its hierarchical structure and semantic DTD tag.

RDF format: Resource Description Framework (RDF) is a new way to define ontology after XOL. It is a resource model always accompanied by a specific URI and extended specific XML-like label to depict the relation and knowledge model between the resources. It not only specifically and strictly expresses the data but also makes the alternation, merging, and inference possible.

According to language protocol of these languages, ontology language can divide into first-order predicate logic language, frame-based language, and concept-role restriction language.

First-order predicate logic language is the most accurate and original language in knowledge representation. The predicate formula is the formula formed by joining some predicates together with the predicate join symbol, like the largest formalized language Cyclo [5] and KIF [6].

Frame-based language is a language that includes the aforehand defining framework and simplified first-order logic language. Owing to excessive strict first-order predict logic and unreadable syntax, Ontolingua and frame logic are developed to remedy this defect.

Concept-role restriction language is an effort that most language currently adopts. This type of language offers a hierarchy way to represent the hyponymy by concept and the individual's signal. It reveals the relationship and value restrictions between different ontologies by role mark, like OML [7].

To note the difference between ontology languages in multiple syntax formats, we will give a detailed introduction for some ontology language with the technical developing route.

\subsection{SHOE (HTML format)}

HTML had covered with a long history before the World Wide Web (WWW) appeared and is one of document standards of Standard Generalized Markup Language (SGML). SGML offers a high standard and complicated description about the document resource. As SGML is hard to learn, use, and realize, researcher put forward the HTML in 1989 after considering the computer's ability. HTML is the mere application of SGML in the WWW times. After few years of great development, HTML is widely known in the web document district. Semantic Web, as the next generation of WWW, is also the use of the HTML syntax in the ontology language. We called this simple HTML ontology language as SHOE. 
SHOE is a specification that describes an extension to HTML, which provides a way to semantically describe important information about HTML or other web documents [1]. It offers a hierarchical classification mechanism for HTML documents and non-HTML documents or subsections of HTML documents. The intent of this specification is to make it possible for user agents, robots, and so on, to gather truly meaningful information about web pages and documents, enabling significantly better search mechanisms and knowledge gathering. Let us take the SHOE as an example; it can divide into two steps as follows:

\section{Define an ontology}

$<$ Ontology "ontology-unique-name" version = "1.0" backward-compatiblewith = "version list">

\section{Use an existing XOL ontology}

$<$ Ontology-extends "ontology-unique-name" version = "Version" backwardcompatible-with = "version list" URL = "location">

This is a simple way to define ontologies containing rules. Ontology simply means an ISA hierarchy of categories and a set of relations between these categories in this SHOE specification. Categories will also inherit relations defined in parent categories. However, this specification does not as yet define any other forms of relationships (transitive closures, inverses, negations, etc.) and use the complicated and human-unreadable Hypertext Markup Language as the basic syntax.

\section{$2.2 \mathrm{KIF}$}

At the same time, first knowledge interchange format was proposed by American National Standard (dpANS). Though many ontology languages are still developed, researcher in Standard University begins to design a language for the intercommunication. Interchange of knowledge or ontology thought out disparate computer systems (different programmers, different languages, and other discrepancy in interknowledge sharing). KIF language is logically comprehensive with declarative semantics.

In addition to these essential features, KIF is designed to maximize the implementability and readability. KIF provides a declarative language for describing knowledge. As a pure specification language, KIF does not include commands for knowledge base query or manipulation.

\subsection{GFP}

GFP is first motivated by the hierarchic framework design of frame-based knowledge representation systems (FRSs) used at the Stanford Knowledge Systems Laboratory for accessing Cyc, KEE, and Epikit [8]. FRSs can contain all of the database systems and knowledge systems or other frame-like projects. It is complementally developed to support knowledge sharing. It specifies a new protocol, Generic Frame Protocol (GFP), for connecting knowledge bases (KBs) in FRSs. In more detail, it provides numbers of operations to formulate a general interface for all of the FRSs. Also, complementary tools were also produced to keep independent and general operation generation. GFP shows well compatibility between different languages, including Java, C (client implementation only), and Common Lisp. Thus, some format conversations of languages are also needed.

\section{$2.4 \mathrm{OKBC}$}

After GFP coming out, OKBC, a new protocol called Open Knowledge Base Connectivity, has taken it up in more implicit knowledge model and knowledge 
operation. It first uses some open ontology systems, such as EcoCyc, GKB Editor, and Ontolingua projects. With 2 years of development, OKBC quickly used in several ontology sharing projects.

OKBC handles the knowledge in more implicit representation formalism, which we called OKBC Knowledge Model in later years. This model not only supports an object-oriented representation of knowledge but also can found the commonly knowledge structure from different KRSs. Therefore, it can serve as an implicit knowledge interlingua by its powerful character in knowledge for all of the systems using OKBC.

\subsection{Ontolingua}

Ontolingua, which accompanies with different ontology languages breaking out, can serve as a basic framework to support open or domain knowledge sharing system. The syntax of Ontolingua definition is based on GFP. It is motivated by the need of Summer Ontology Project, a pilot study in which researchers from several groups and institutions met weekly to design ontology of terms used in modeling electromechanical devices. Figure 1 depicts the structure of Ontolingua.

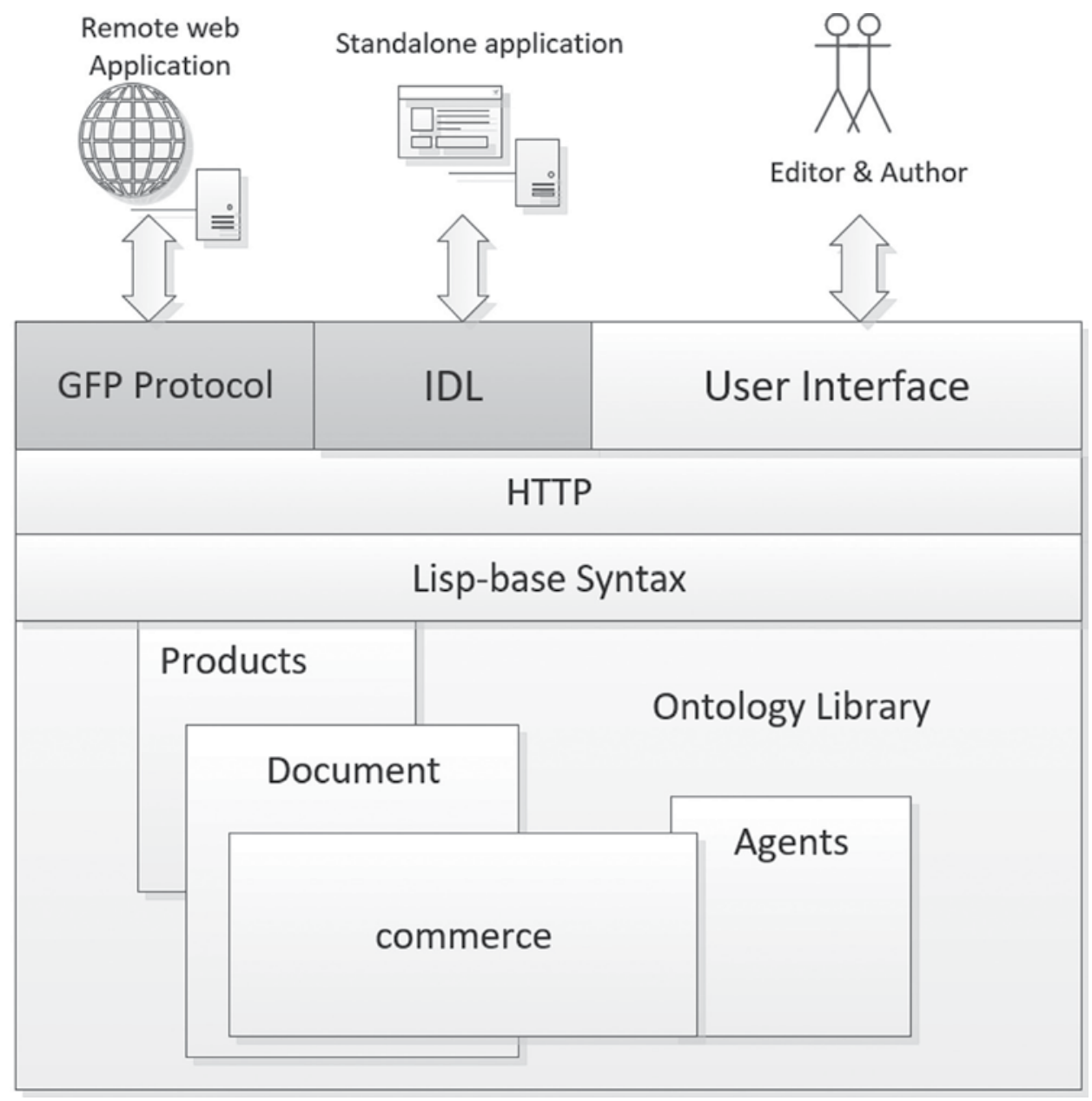

Figure 1.

The structure of Ontolingua. 


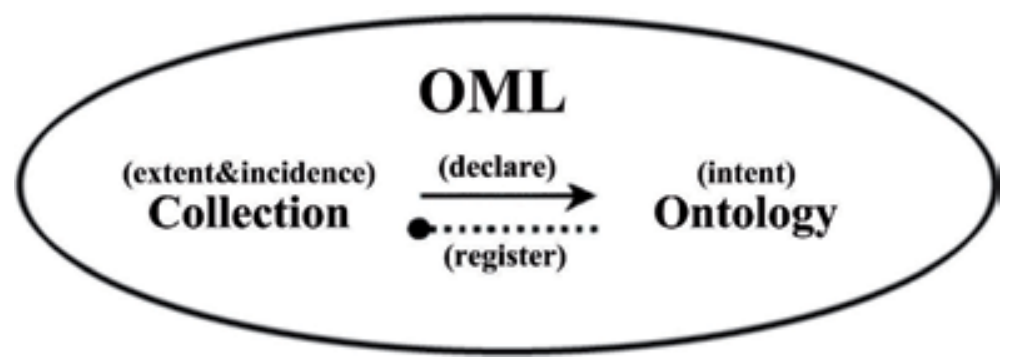

Figure 2.

The structure of $O M L$.

At first, authors and editors will publish their ontology or maintain an ontology with a HTTP protocol connection. Users may access the different defined ontologies in Ontology Library, for example, ontologies, product ontologies, document ontologies, commerce ontologies, and agent knowledge. Beside the ontology editors or editors, some remote application may also need to duly connect this ontology library in GFP protocol to exchange or manipulate ontology. When it comes to standalone application, interface definition language with a specific and functional method will retrieve an ontology batch from ontology library. Ontolingua keeps a balance in generalization expressed GRP protocol and selectivity in some standalone application by own interface definition language (IDL).

Ontolingua can thereby be shared by multiple user and research groups using their own favorite representation systems and can be easily ported from system to system. The syntax of Ontolingua definition is simplified with some class name, argument, and documentation string.

\subsection{OML}

$\mathrm{XOL}$ is more similar to Ontolingua. However, Ontolingua using $\mathrm{OKBC}$ is frame-based design and less semantic expression. At the same time, a separate set of researchers is pursuing a concept-role restriction language-OML. Ontology markup language $(\mathrm{OML})$ is an adaptive change language based on SHOE. Earlier versions of OML were basically an Extensive Markup Language (XML) translation of the SHOE language with suitable changes and improvements. Common elements existing in OML can be described by paraphrasing the SHOE documentation to some degree. Now OML is highly RDF Schemas compatible, although it has own solution within the namespace problem. More importantly, OML has incorporated own elements and expressiveness of conceptual graphs. As shown in Figure 2, by declaring and registering operation, OML can be seen as a bridging connection between Ontology and Collection, which reflects more extent and incidence of ontology.

\subsection{An ontology exchange language in XML format: XOL}

With the development of the relative language, protocol and definition, and the need of an Evaluation of Ontology Exchange Languages for bioinformatics, several researchers on the evaluation team are currently developing a specification of XML expression of Ontolingua using OKBC, while a separate set of researchers is pursuing a frame-based version of OML. However, Ontolingua first uses a Lisp-based syntax (rather than HTML-based or XML-based), which leads to become hard to develop and maintain, though the semantics of OKBC-Lite are extremely similar to the semantics of Ontolingua. At this background, XOL was first published in 1999. 


\section{The usages of XOL}

The usages of XOL are based on frame-based approach. In this part, we will introduce the constituent part and its usage mode.

\subsection{Basic data type}

- Integer

- Floating point numbers

- Double-precision floating point numbers

- Strings

- Boolean

- Name of class

\subsection{Classes}

Classes are composed of entities. Entity that is not the class but an instance of a class or multiple classes is said to be Individual. Classes and Individual distinguish by whether entity is a class of another entity or not. Class entity male or class entity female is the subclass of another class entity human, a man called Joe is an Individual entity of the class entity. The following Class is the basic Class description defined in the OKBC-Lite (Table 1).

\subsection{Slots on slots}

Slot is common property of each class or instance. The attribute "Documentation" of class has an introduction to this class. When it comes to the specific $\mathrm{KB}$, slot may divide into "own slot" in this KB or "template slot" inheriting from class.

Slots on slots, as shown in Table 2, are the several restrictions or declarations defined to this slot. Although it may be inherited from other KB or class, restriction or declaration on slots in this XOL file is exclusive.

\begin{tabular}{llll}
\hline Name & Description & Name & Description \\
\hline THING & $\begin{array}{l}\text { The root of the class } \\
\text { hierarchy } \\
\text { The superclass of every class }\end{array}$ & SYMBOL & $\begin{array}{l}\text { The class of all symbols } \\
\text { A subclass of THING }\end{array}$ \\
\hline CLASS & The class of all classes & INDIVIDUAL & $\begin{array}{l}\text { The class of all entities that are not } \\
\text { classes }\end{array}$ \\
\hline NUMBER & $\begin{array}{l}\text { The class of all numbers } \\
\text { A subclass of INDIVIDUAL }\end{array}$ & INTEGER & A subclass of NUMBER \\
\hline STRING & $\begin{array}{l}\text { The class of all text strings } \\
\text { A subclass of INDIVIDUAL }\end{array}$ & LIST & $\begin{array}{l}\text { The class of all lists } \\
\text { A subclass of INDIVIDUAL }\end{array}$ \\
\hline
\end{tabular}

Table 1.

The classes of XOL [2]. 


\begin{tabular}{|c|c|}
\hline Name & Function \\
\hline DOMAIN & $\begin{array}{l}\text { Specifies the domain of the binary relation represented by } \\
\text { a slot frame }\end{array}$ \\
\hline SLOT-VALUE-TYPE & $\begin{array}{l}\text { Specifies the classes of which values of a slot must be an } \\
\text { instance }\end{array}$ \\
\hline SLOT-INVERSE & Specifies the inverse relation for a slot \\
\hline SLOT-CARDINALITY & $\begin{array}{l}\text { Specifies the exact number of values that may be asserted } \\
\text { for a slot for entities in the slot's domain }\end{array}$ \\
\hline SLOT-MAXIMUM-CARDINALITY & $\begin{array}{l}\text { Specifies the maximum number of values that may be } \\
\text { asserted for a slot for entities in the slot's domain }\end{array}$ \\
\hline SLOT-MINIMUM-CARDINALITYNUMERIC & $\begin{array}{l}\text { Specifies the minimum number of values for a slot for } \\
\text { entities in the slot's domain }\end{array}$ \\
\hline SLOT-NUMERIC-MAXIMUM & $\begin{array}{l}\text { Specifies a lower bound on the values of a slot for entities } \\
\text { in the slot's domain }\end{array}$ \\
\hline SLOT-NUMERIC-MINIMUM & $\begin{array}{l}\text { Specifies an upper bound on the values of a slot for entities } \\
\text { in the slot's domain }\end{array}$ \\
\hline SLOT-COLLECTION-TYPE & $\begin{array}{l}\text { Specifies whether multiple values of a slot are to be treated } \\
\text { as a set, list, or bag }\end{array}$ \\
\hline
\end{tabular}

Table 2.

The slots on slots of $X O L$.

\begin{tabular}{|c|c|c|c|}
\hline Name & Description & Name & Description \\
\hline VALUE-TYPE & $\begin{array}{l}\text { Value can be class or } \\
\text { multiclass or set of } \\
\text { value }\end{array}$ & $\begin{array}{l}\text { MINIMUM- } \\
\text { CARDINALITY }\end{array}$ & $\begin{array}{l}\text { The class of all symbols } \\
\text { A subclass of THING }\end{array}$ \\
\hline INVERSE & $\begin{array}{l}\text { Describe the slot } \\
\text { relation is reverse } \\
\text { and value is reverse } \\
\text { slot }\end{array}$ & NUMERIC-MINIMUM & $\begin{array}{l}\text { Specifies lower bound on } \\
\text { the number-type values } \\
\text { of slot }\end{array}$ \\
\hline CARDINALITY & $\begin{array}{l}\text { Specifies the exact } \\
\text { number of values } \\
\text { asserted for a slot }\end{array}$ & $\begin{array}{l}\text { NUMERIC- } \\
\text { MAXIMUM }\end{array}$ & $\begin{array}{l}\text { Specifies upper bound on } \\
\text { the number-type values } \\
\text { of a slot }\end{array}$ \\
\hline $\begin{array}{l}\text { MAXIMUM- } \\
\text { CARDINALITY }\end{array}$ & $\begin{array}{l}\text { Specifies the } \\
\text { maximum number } \\
\text { of values asserted } \\
\text { for a slot }\end{array}$ & COLLECTION-TYPE & $\begin{array}{l}\text { Specifies whether } \\
\text { multiple values of a slot } \\
\text { are to be treated as set/ } \\
\text { list/bag. }\end{array}$ \\
\hline
\end{tabular}

Table 3.

The facet of $X O L$.

\subsection{Acceptable facet}

Different with slots on slots, facet is a restriction on the value of slot of individual. For instance, facet VALUE-TYPE and facet NUMERIC-MINIMUM describe the type of value of a slot and the minimum of value of a slot. Table 3 is the acceptable facet defined in OKBC-Lite.

Facet can also divide into two parts:

- Own Facet (only state on current class or current KB)

- Template Facet (inherit from another class) 


\section{XOL example}

Every XOL file must start with the following XML tab in the beginning. $<$ ? xml version="1.0"? ><!DOCTYPE module SYSTEM "module.dtd">

As the whole KB's first description, 'module section' will illuminate some information (name, type of DB, in which package, etc.) about this KB.

$<$ Module $>/{ }^{*}$ Every XOL file will start with a module mark ${ }^{*} /$

$<$ Name $>$ name of this $\mathrm{KB}</$ Name $>$

$<$ kb-type $>$ which existing kb type $</$ kb-type $>$

$<$ package $>$ self-defined package name $<$ /package $>$

The second section is 'class section.' In this section, we will introduce all these KB's classes or inherit from other class by the tag 'subclass-of' or 'instance-of.'

$<$ Class $>$

$<$ Name $>$ name of class $<$ /Name $>$

$<$ Documentation $>$ String-type description $</$ Documentation $>$

[Other-option-slot] (Subclass-of $\mid$ instance-of $\mid$ etc.)

$</$ Class $>$

The third section is 'slot section,' which declares all slots in the class and slots existing slot value in an individual, such as the class-name, class-documentation, and other-option slot.

$<$ Slot $>$.

$<$ Name $>$ name of own slot or template slot $</$ name $>$

$<$ Documentation $>$ description $</$ documentation $>$

$<$ Domain (or other slots on slots in Table 2) $>.<$ /Domain $>$

$<$ /Slot $>$

The last section generally is 'individual section.' It contains all instances and their values in each slot. Also, it declares restriction of value of slot and slot-values.

$<$ Individual $>$

$<$ Name $><$ /name $>$

$<$ instance-of $><$ /instance-of $>$

$<$ Slot-values $>$

$<$ Name $>\ldots<$ /name $>$

$<$ Values $>\ldots<$ /values $>$

$<$ value-type (or facet in Table 3 ) $>\ldots</$ value-type $>$

$</$ slot-values $>$

$<$ individual>

At last, remember that XOL file must use $<$ /model $>$ to note the ending.

\section{Future developments: OIL}

Framework representation is to express the concepts, instances, classes, and relationships used in ontology in the form of framework. XOL is such a framework method-based ontology representation language. Unlike the rich expressions in logic-based approach, XOL leads to the deficiency in reasoning ability. The main differences stem from the fact that frames generally provide quite a rich set of primitives but impose very restrictive syntactic constraints on how primitives can be combined and on how they can be used to define a class.

Due to the deficiency of XOL in grammatical reasoning and the continuous development of DL notation, another new ontology interactive language OIL is defined [9]. It is not only an ontology description language but also a frame-based web language and an XML and RDF compatible ontology language. Its appearance unifies the characteristics of traditional ontology language and endows the new object into the inference layer. 


\title{
6. Conclusion
}

The emergence of XOL was inspired by existing ontology and protocol, for example, SHOE, KIF, GFP, OKBC, and so on. XOL is a bridge language, which let the ontology using frame-based approach can be expressed in a simplifier way during the XML file. By the human-readable XML and unified Label limitation, it can use as an ontology exchange language during the cross application, which allows to obey the use of the XOL (in fact is OKBC-Lite) restriction. However, lacking of inferential capability and more logical expression, XOL was replaced by the subsequent ontology language OIL considering the multilanguage and more logic restriction that enable to validate ontology.

This chapter gives an overlook of XOL from the historical development across the different ontology languages. Note that $\mathrm{XOL}$ is not the first language in defining the ontology language for exchange date. It merely complements XML syntax and uses a simple frame-based OKBC protocol. However, it still lacks more compatible with multiple ontology protocols and different syntax. Without the more consideration into inference, ontology quickly would replace by stronger ontology system.

We also found that while designing a better widely used ontology language, we should keep a right balance between generality and specificity or between compatibility and limitation. We will focus more on the result comparison between different ontology methods and the humanity background within different languages.

\author{
Author details \\ Jinta Weng, Jing Qiu* and Ying Gao \\ Guangzhou University, China \\ *Address all correspondence to: qiujing.ch@gmail.com
}

IntechOpen

(C) 2020 The Author(s). Licensee IntechOpen. This chapter is distributed under the terms of the Creative Commons Attribution License (http://creativecommons.org/licenses/ by/3.0), which permits unrestricted use, distribution, and reproduction in any medium, provided the original work is properly cited. (cc) BY 


\section{References}

[1] Hendler J, Berners-Lee T. From the semantic web to social machines: A research challenge for AI on the world wide web. Artificial Intelligence. 2010;174(2):156-161. DOI: 10.1016/j. artint.2009.11.010

[2] Karp R, Chaudhri V, Thomere J. XOL: An XML-based ontology exchange language. Version 0.4. 2002. Available from: http://www.ai.sri.com/ pkarp/xol [Accessed: 18 May 2019]

[3] Chaudhri VK, Farquhar A, Fikes R, Karp PD, Rice JP. OKBC: A programmatic foundation for knowledge base interoperability. In: Proceedings of the National Conference on Artificial Intelligence. 1998

[4] Gruber TR. Ontolingua: A mechanism to support portable ontologies. Knowledge Systems Laboratory. Stanford, CA: Computer Science Department, Stanford University; 1992:94305

[5] Lenat DB, Guha RV. Building Large Knowledge-Based Systems; Representation and Inference in the Cyc Project. 1st ed. USA: Addison-Wesley Longman Publishing Co.; 1989. DOI: $10.5555 / 575523$

[6] Michael R. Genesereth. Knowledge interchange format [Internet]. 1998. Available from: http://logic.stanford.edu/ kif/dpans.html [Accessed: 18 May 2019]

[7] Kent RE. Conceptual knowledge markup language: An introduction. NETNOMICS. 2000;2(2):139-169. DOI: 10.1023/a:1019186729572

[8] Vinay K. Chaudhri, et al. Generic Frame Protocol (GFP). 1997. Available from: http://www.ai.sri.com/ gfp/ [Accessed: 18 May 2019]

[9] van Harmelen F, Horrocks I. FAQs on OIL: The ontology inference layer. IEEE Intelligent Systems. 2000;15(6):69-72 



\title{
An Ontology-Based Approach to Diagnosis and Classification for an Expert System in Health and Food
}

\author{
Friska Natalia, Dea Cheria and Santi Surya
}

\begin{abstract}
In this chapter, we will discuss how to make an ontology-based expert system easy to use and apply to community sustainability issues without pay. Ontology itself plays an essential role in the diversity of knowledge and management methods that can simplify communication between expert domains and users. The scope of this study is health and food, which is expected to help people realize the disturbances they experience. In this chapter, we will discuss two cases: (i) determine the depressive disorder a person has based on their health condition and (ii) determine the type and variant of rice according to needs. Ontology is a method used in research that can be structured and systematic real-world representation that is equal and provides a reference model. The results of this study are an expert system model and mobile applications to help users overcome the problems in the health and food fields with the ontology method. The objective of this study is to develop the application based on the ontology method to make it easy for people to find information on expert systems.
\end{abstract}

Keywords: expert system, modelling, ontology, depressive disorder, variant of rice

\section{Introduction}

Ontology matching is a field of research that is in high demand today, where information exchange and reuse of knowledge are essential topics in the development of the ontology; this is one solution to the problem of semantic heterogeneity. Matching ontology aims to find correspondence between entities in the semantic ontology. In this study, we will discuss how to make an ontology-based expert system to be easily used by the community without pay. Ontology itself plays a vital role in the diversity of knowledge and the way it is regulated. Ontology is a structured and systematic equivalent real-world representation. Moreover, ontology also provides a referral model that can simplify communication between expert domains and improve understanding and information sharing. There are several previous research related to ontology. The first paper describes a knowledge system for improving RFID recognition by using fuzzy ontology [1]. The second paper shows the fuzzy ontology with fuzzy concepts is an extension of the domain ontology with crisp concepts [2]. The third paper concerns to integrate ontologies from food, health, and nutrition domains to help the personalized information systems to 
retrieve food and health recommendations based on the user's health conditions and food preferences [3]. The fourth paper provides advancement to the research of diabetes diagnosis using CBR algorithm [4]. The fifth paper proposes a search based on multiple ontologies to make information retrieval efficient. It rewrites the user query by adding semantic information, after consulting multiple ontologies [5]. The other paper is to integrate ontologies from food, health, and nutrition domains to help the personalized information systems to retrieve food and health recommendations based on the user's fitness conditions and food preferences [6].

In this study, the object of research to be used is a ubiquitous object in the community, namely, health and food. Ontology itself plays an essential role in the diversity of science and how to regulate it. Cytology is a real structured and systematic representation of the world. Moreover, ontology also provides a reference model that can simplify communication between domain experts and improve understanding and information sharing.

Many similar studies have used the decision tree so that its use is more familiar than ontology. However, in this study, classification ontology does not require elimination or precise calculations to be able to take a conclusion, as did the decision tree, because the results are taken from predetermined criteria. Although ontology cannot be flexible in choosing criteria for different outcomes, the classification ontology method is more directed at relationships within each entity rather than elimination based decision-making.

(i) Health is a very complicated problem, which is the result of various natural and human-made environmental problems. The coming of a disease is something that cannot be rejected, although sometimes it can be prevented or rejected. The concept of health or sickness is truly undivided and universal because other factors influence greatly, especially sociocultural factors. Therefore, it is crucial to have thoughts about the concepts of health and illness. If the idea of the concept is based on the correct concept, then the community will also find the right alternative to solve their health problems. The type of disease in this world is comprehensive. Some include common diseases, but some include diseases that are quite difficult to avoid. One of them is an allergic disease. (ii) Food will be discussed for the selection of rice to be consumed in fulfilling daily intake depending on consumer tastes. Rice is one of the essential cereals needed by humans for consumption. Even though some countries in the world have basic needs to switch to wheat products, the Indonesian people still rely heavily on rice as a basic necessity to meet their daily needs.

The results of this study are an expert system model and application in the form of a mobile application using ontology created by Protégé ontology web language (OWL) to help users. This research is also expected to help users know the types of depressive disorders and recognize the criteria of someone experiencing the depressive disorder in the health sector and help to sort and select the variants and types of rice that best suit the needs of users with the ontology method. The method that will be used is ontology because ontology provides potential compatibility and chooses the results that are most suitable according to the needs and criteria given. Also, ontology is a way to describe the meaning and relationship of a term. The description can help computer systems to understand the terms used more efficiently. Thus the needs can be sorted and chosen appropriately without just perception.

Based on the above problems, these are the objectives of this study:

1. Know the application of the ontology method in making expert systems.

2. Make it easy for people to find information on expert systems. 


\section{Problem statement and research methodology}

In this study, there are two scenarios to be discussed: health and food.

i. Health: The Indonesian people still lie about mental disorder, so what usually happens is that they isolate patients. Activities, social life, the rhythm of work, and relationships with families are disrupted due to symptoms of anxiety, depression, and psychosis. Someone with any mental disorder must get treatment immediately. Delay in treatment will be more detrimental to sufferers, families, and society. In this study, mental health ontology (for cases of depressive disorder) will be made based on the types and provide any information that characterizes a person suffering from a depressive disorder. The design will use Protégé, with the latest data made by the American Psychiatric Association and implemented into an Android-based mobile application, which is expected to help people realize the disturbances they experience and get immediate treatment.

ii. Food: In this scenario in the selection of rice, there is no specific reference for making choices. Difficulties are also felt when there are no specific benchmarks based on experts or the right knowledge to determine the compatibility between processed and rice types. Besides, the application of ontology can also be applied in the world of food and medicine. This makes it interesting to apply the ontology to one aspect of food, in this study, rice, intending to create an Android-based model and expert system application to sort and select the variants and types of rice that best suit the needs of users with the ontology method. Because ontology provides potential compatibility and choices, the results that are most suitable according to the needs and criteria are given. Thus the needs can be sorted and chosen appropriately, not only with perception.

The method used in this study is the classification ontology method; besides because it is the method most often used in similar studies, this method shows more the real identity and relationship in each entity compared to the decision tree which leads to eliminating unnecessary calculations. Ontology consists of basic terms, the relationship between those terms, and rules that incorporate them. The ontology can become knowledge that can be shared and used in multiple applications. The reason for using this method is because it is the most suitable way to perform data groupings and interclass entity relationships. Considering the object of this study, then the method used in this study is the classification ontology method because this study does not require the elimination of particular calculations and the classification ontology method is more directed at relationships in each entity rather than elimination-based decision-making.

In this research, the rapid application development (RAD) system development method will be used to develop mobile applications because this method is commonly used for making relatively short systems. The stages in the rapid application development method are [7-9]:

\section{Stage requirement planning}

At this stage, a plan is carried out to determine what data is needed for system requirements.

\section{Stage design}

At this stage, a temporary design is made that focuses on presentation. 


\section{Implementation phase}

At this stage, the results will be translated into the appropriate programming language and then be tested before being disseminated.

\section{Design and implementation}

\subsection{First scenario}

Requirement planning, in the system requirement: the system can select for user data search purposes, and the system can do searching or filtering to find the right data. In data requirement: All data were from the Diagnostic and Statistical Manual of Mental Disorders Fifth Edition, which was created by a selection of selected psychiatrists from the American Psychiatric Association. In this study, only a limited part of the depressive disorder and diagnostic criteria in it.

Depressive disorder is the presence of sad, empty, or irritable mood, accompanied by physical and cognitive changes that impact function [10]. Based on that, we can categorize it into eight types: Someone who has disruptive mood dysregulation disorder does not experience depressed mood, but becomes more irritable and more sensitive, often has problems with his mood, has experienced symptoms for more than 12 months, is not affected by drugs, has never had medical treatment, is not coming month (for women), and does not have other psychological diagnoses. Also, the person exhibits persistent irritability and frequent episodes of extreme verbal and behavioral dyscontrol toward people or property out of proportion to the situation and is inconsistent with developmental level occurring on average three or more times per week. Someone who has major depressive disorder experiences depressed mood, does not become more irritable and more sensitive, often has problems with his mood, has experienced symptoms for more than 2 weeks, is not under the influence of drugs, has never had medical treatment, is not coming month (for women), and does not have other psychological diagnoses. Someone who has persistent depressive disorder experiences depressed mood, does not become more irritable or more sensitive, often has problems with his mood, has experienced symptoms for more than 2 years, is not under the influence of drugs, has never had medical treatment, is not coming month (for women), and does not have other psychological diagnoses. Someone who has premenstrual dysphoric disorder experiences depressed mood, becomes more irritable or more sensitive, often has problems with his mood, does not know when to start feeling the problem, is not under the influence of drugs, has never had medical treatment, is on the moon (for women), and does not have other psychological diagnoses. In all of the cycles, symptoms present in the final week before menses, start to improve within a few days after onset, and become minimal or absent in the week postmenses. Someone who has substance-/medication-induced depressive disorder experiences depressed mood, does not become more irritable or more sensitive, often has problems in his mood, has experienced symptoms for more than 1 month, is in the influence of drugs, is undergoing medical treatment, does not have moderate menstruation (for women), and does not have other psychological diagnoses. Someone who has depressive disorder due to another medical condition experiences depressed mood, does not become more irritable and more sensitive, often has problems with his mood, does not know when to start feeling the problem, is not influenced by drugs, has undergone medical treatment, is not having menstruation (for women), and does not have other psychological diagnoses. Someone who has other specified disorder and unspecified depressive disorder experiences depressed mood, becomes 
more irritable or more sensitive, rarely has problems with his mood, has experienced symptoms within a period of time but not daily, is not influenced by drugs, has never had medical treatment, is not having a period (for women), and maybe having another psychological diagnosis.

Table 1 shows the choice of whether the user experiences a depressed mood. Depressed mood includes poor appetite or overeating, insomnia or hypersomnia, low energy or fatigue, low self-esteem, difficulty in concentrating or difficulty in making decisions, despair, and anxiety.

In Table 2, the more the user is accessible to anger means the user is more accessible to emotion than he should. It is easier to get angry because he is more sensitive to ordinary things.

Table 3 shows the problem in the mood is divided into three, such as, all the time which means the problem in the mood is experienced at any time; often which means the problem in the mood is experienced almost every day, but not every time; while rarely can mean the problem in the mood is only experienced occasionally and not necessarily every day, or only at certain times.

Table 4 describes the effect of narcotics on users. If a user is a drug user when feeling symptoms of depressive disorder, then the user may be included in substance-/medication-induced depressive disorder or even not included in any depressive disorder.

Medical treatment can be seen in Table 5. If the user is undergoing treatment, there is a possibility that the user will enter into substance-/medication-induced depressive disorder, or if the user has undergone treatment, there is a possibility

\begin{tabular}{lll}
\hline Number & Disorder name & Depressed mood \\
\hline 1 & Disruptive mood dysregulation disorder & No \\
\hline 2 & Major depressive disorder & Yes \\
\hline 3 & Persistent depressive disorder (dysthymia) & Yes \\
\hline 4 & Premenstrual dysphoric disorder & Yes \\
\hline 5 & Substance-/medication-induced depressive disorder & Yes \\
\hline 6 & Depressive disorder due to another medical condition & Yes \\
\hline 7 & Other specified depressive disorder & Yes \\
\hline 8 & Unspecified depressive disorder & Yes \\
\hline
\end{tabular}

Table 1.

Depressed mood.

\begin{tabular}{lll}
\hline Number & Disorder name & Easy to get angry \\
\hline 1 & Disruptive mood dysregulation disorder & Yes \\
\hline 2 & Major depressive disorder & No \\
\hline 3 & Persistent depressive disorder (dysthymia) & No \\
\hline 4 & Premenstrual dysphoric disorder & Yes \\
\hline 5 & Substance-/medication-induced depressive disorder & No \\
\hline 6 & Depressive disorder due to another medical condition & No \\
\hline 7 & Other specified depressive disorder & Yes \\
\hline 8 & Unspecified depressive disorder & Yes \\
\hline
\end{tabular}

Table 2.

Easy to get angry. 


\begin{tabular}{lll}
\hline Number & Disorder name & Mood problem \\
\hline 1 & Disruptive mood dysregulation disorder & Often \\
\hline 2 & Major depressive disorder & All the time \\
\hline 3 & Persistent depressive disorder (dysthymia) & All the time \\
\hline 4 & Premenstrual dysphoric disorder & Often \\
\hline 5 & Substance-/medication-induced depressive disorder & Often \\
\hline 6 & Depressive disorder due to another medical condition & Often \\
\hline 7 & Other specified depressive disorder & Rarely \\
\hline 8 & Unspecified depressive disorder & Rarely \\
\hline
\end{tabular}

Table 3 .

Mood problem.

\begin{tabular}{lll}
\hline Number & Disorder name & In the influence of drugs \\
\hline 1 & Disruptive mood dysregulation disorder & No \\
\hline 2 & Major depressive disorder & No \\
\hline 3 & Persistent depressive disorder (dysthymia) & No \\
\hline 4 & Premenstrual dysphoric disorder & No \\
\hline 5 & Substance-/medication-induced depressive disorder & Yes \\
\hline 6 & Depressive disorder due to another medical condition & No \\
\hline 7 & Other specified depressive disorder & No \\
\hline 8 & Unspecified depressive disorder & No \\
\hline
\end{tabular}

Table 4.

The influence of drugs.

\begin{tabular}{lll}
\hline Number & Disorder name & In medical treatment \\
\hline 1 & Disruptive mood dysregulation disorder & No \\
\hline 2 & Major depressive disorder & No \\
\hline 3 & Persistent depressive disorder (dysthymia) & No \\
\hline 4 & Premenstrual dysphoric disorder & No \\
\hline 5 & Substance-/medication-induced depressive disorder & Is undergoing \\
\hline 6 & Depressive disorder due to another medical condition & Ever undergoing \\
\hline 8 & Other specified depressive disorder & No \\
\hline
\end{tabular}

Table 5.

Medical treatment.

that the user will enter into depressive disorder due to another medical condition, or even depressive disorder.

Table 6 is only for women, and if it meets the criteria, there is a possibility that the user includes premenstrual dysphoric disorder, but it may not be included in any depressive disorder.

Table 7 has shown the length of time for how long a person experiences symptoms of depressive disorder, starting from 2 weeks, 1 month, 12 months, 2 years, not 
An Ontology-Based Approach to Diagnosis and Classification for an Expert System in Health... DOI: http://dx.doi.org/10.5772/intechopen.88180

long ago for those who experience infrequently, or do not know, because they feel they have enough old but only certain moments.

Table 8 describes that one of the main requirements in depressive disorder is not having another psychological diagnosis. Several other mental disorders have characteristics similar to depressive disorder; if the user feels that he has another psychological diagnosis, then maybe the user is included in other specified

\begin{tabular}{lll}
\hline Number & Disorder name & In the menstruation time \\
\hline 1 & Disruptive mood dysregulation disorder & No \\
\hline 2 & Major depressive disorder & No \\
\hline 3 & Persistent depressive disorder (dysthymia) & No \\
\hline 4 & Premenstrual dysphoric disorder & Yes \\
\hline 5 & Substance-/medication-induced depressive disorder & No \\
\hline 6 & Depressive disorder due to another medical condition & No \\
\hline 7 & Other specified depressive disorder & No \\
\hline 8 & Unspecified depressive disorder & No \\
\hline
\end{tabular}

Table 6.

In the menstruation time.

\begin{tabular}{lll}
\hline Number & Disorder name & How long \\
\hline 1 & Disruptive mood dysregulation disorder & 12 months \\
\hline 2 & Major depressive disorder & 2 weeks \\
\hline 3 & Persistent depressive disorder (dysthymia) & 2 years \\
\hline 4 & Premenstrual dysphoric disorder & Do not know \\
\hline 5 & Substance-/medication-induced depressive disorder & 1 month \\
\hline 6 & Depressive disorder due to another medical condition & Do not know \\
\hline 7 & Other specified depressive disorder & Not long ago \\
\hline 8 & Unspecified depressive disorder & Not long ago \\
\hline
\end{tabular}

Table 7.

How long.

\begin{tabular}{lll}
\hline Number & Disorder name & Have other diagnoses \\
\hline 1 & Disruptive mood dysregulation disorder & No \\
\hline 2 & Major depressive disorder & No \\
\hline 3 & Persistent depressive disorder (dysthymia) & No \\
\hline 4 & Premenstrual dysphoric disorder & No \\
\hline 5 & Substance-/medication-induced depressive disorder & No \\
\hline 6 & Depressive disorder due to another medical condition & No \\
\hline 8 & Other specified depressive disorder & Could have \\
\hline
\end{tabular}

Table 8.

Have other psychological diagnosis. 


\begin{tabular}{lll}
\hline Number & Rice type & Description \\
\hline 1 & White rice & White rice is commonly consumed \\
\hline 2 & Dark Brown Rice & Similar to white rice but slightly brownish \\
\hline 3 & Brown rice & Has a reddish outer layer \\
\hline 4 & Black rice & Rice that is rather blackish \\
\hline 5 & White glutinous rice & Glutinous rice that is thick and white \\
\hline 7 & Black glutinous rice & Blackish sticky rice \\
\hline 9 & Parboiled rice & Rice that is soaked in warm water first \\
\hline 10 & Mixed rice & A mixture of several types of rice \\
\hline 11 & Basmati rice & Middle Eastern rice \\
\hline
\end{tabular}

Table 9.

Brief description of rice types.

depressive disorder or unspecified depressive disorder or even not including depressive disorder.

\subsection{Second scenario}

In order to create a system, a thorough analysis of system requirements is needed to make a great system. The analysis was carried out on interview data that had been conducted so far to produce a proper application which ran smoothly according to the initial needs. In this scenario, interviews were conducted to obtain the data needed. From the results of interviews conducted with the three speakers, one of the resource persons summarized the knowledge of the three experts, including himself, to make a rule in determining the type of rice that matches the processed food, along with the brand according to the specified criteria. Criteria are determined based on information from experts regarding the type of rice according to processing. Table 9 shows the types of rice found in Indonesia based on the interviews.

The following are brands of rice that sell these types:
a. Myrice
b. Parakeet
c. Basket
d. Louhan
e. Goldrice Red
f. Goldrice Green
g. King rice
h. Swallow 
An Ontology-Based Approach to Diagnosis and Classification for an Expert System in Health... DOI: http://dx.doi.org/10.5772/intechopen.88180

i. VIP

j. Panda

k. Three guava

1. Cap

m. Penguin

n. BMW

o. Guci Mas

p. Flower Stamp

The following are processed rice commonly made by consumers in the rice stores according to the seller's knowledge:
a. Bakcang
b. Porridge
c. Black glutinous porridge
d. Gyudon
e. Egg-crust
f. Lemper
g. Lontong/ketupat
h. Rice

i. Baked rice

j. Fried rice

k. Gudeg rice

1. Corn rice

m. Yellow rice

n. Liwet rice

o. Team rice

p. Uduk rice

q. Sushi 
Table 10 shows the criteria for rice used:

The following is a table of matches between the types, criteria, and brands of rice with processed rice; the original table sent by the guest speaker is in the Appendix.

Explanation of Table 11:

$\mathrm{G}=$ Glutinousness. $(+)$ means it is more sticky or contains water. (-) means it is slightly watery or sticky.

$\mathrm{L}=$ Length. $(+)$ means more oblong. (=) means more rounded.

$\mathrm{A}=$ Aroma. $(+)$ means more fragrant. $(-)$ means less fragrant.

$\mathrm{R}=$ Taste. $(+)$ means sweeter. $(-)$ means more acidic.

$=$ means having these criteria within normal limits.

\begin{tabular}{lll}
\hline Number & Criteria & Description \\
\hline 1 & Glutinousness & Rice stickiness level \\
\hline 2 & Taste & The resulting taste (more acidic or sweet) \\
\hline 3 & Aroma & The fragrance level of rice \\
\hline 4 & Length & The length and shape of rice (somewhat oval or rather round) \\
\hline
\end{tabular}

Table 10.

Brief description of rice criteria.

\begin{tabular}{|c|c|c|c|c|c|c|c|}
\hline \multirow{2}{*}{$\begin{array}{l}\text { Number } \\
1\end{array}$} & \multirow[t]{2}{*}{ Processed } & \multirow[t]{2}{*}{ Types of rice } & \multicolumn{4}{|c|}{ Criteria } & \multirow[t]{2}{*}{ Brand of rice } \\
\hline & & & G & $\mathbf{L} \quad$ & A 1 & $\mathbf{R}$ & \\
\hline 2 & Bakcang & White rice, glutinous rice & + & & & $\sqrt{ }$ & Myrice, Penguin \\
\hline 3 & Porridge & White rice & & + & & $\sqrt{ }$ & Flower Stamp \\
\hline 4 & $\begin{array}{l}\text { Black sticky rice } \\
\text { porridge }\end{array}$ & Black glutinous rice & & & & $\sqrt{ }$ & Parakeet, Bakul \\
\hline 5 & Gyudon & Mixed rice, Japan & + & & & $\sqrt{ }$ & Louhan, Goldrice Green \\
\hline 6 & Egg-crust & & - & & - & & Swallow \\
\hline 7 & Lemper & White glutinous rice & + & & - & & $\begin{array}{l}\text { Swallow, King rice, } \\
\text { Goldrice Green }\end{array}$ \\
\hline 8 & Lontong/ketupat & White rice & + & & & $\sqrt{ }$ & Myrice, King rice \\
\hline 9 & Rice & (All) & $\sqrt{ }$ & $\sqrt{ }$ & $\sqrt{ }$ & $\sqrt{ }$ & (All) \\
\hline 10 & Roasted rice & $\begin{array}{l}\text { White rice, parboiled rice, } \\
\text { brown rice }\end{array}$ & & & + & $\sqrt{ }$ & Goldrice Red, VIP \\
\hline 11 & Fried rice & White rice, red rice & - & & & $\sqrt{ }$ & Panda, BMW, Penguin \\
\hline 12 & Gudeg rice & White rice & + & & & $\sqrt{ }$ & Three Guava, Capit \\
\hline 13 & Corn rice & Basmati rice & + & & + & $\sqrt{ }$ & Three Guava, Capit \\
\hline 14 & Yellow rice & White rice & - & & - & $\sqrt{ }$ & Panda, BMW, Penguin \\
\hline 15 & Liwet rice & White rice & + & & & $\sqrt{ }$ & Three Guava, Capit \\
\hline 16 & Team rice & White rice & + & & & $\sqrt{ }$ & Three Guava, Capit \\
\hline 17 & Uduk rice & White rice & - & & & $\sqrt{ }$ & Panda, BMW, Penguin \\
\hline 18 & Sushi & Japanese rice, mixed rice & + & & & $\sqrt{ }$ & Louhan, Goldrice Green \\
\hline
\end{tabular}

Table 11.

Processed compliance tables with types, criteria, and brand of rice. 


\section{Results}

Figure 1 describes a flowchart design used in the process of making this system or application. First, the collected data will be analyzed and will be used as a reference in making classes and subclasses on ontology, and the process of creating classes and subclasses will involve the use of Protégé tools. Then the next step is to determine the property. At this stage, we will determine the property object and data property, which will be needed as attributes and relations of each data. The first property object is created based on the class and subclass that were created before, and each class will have its data property.

After that create a data property; this time the creation will be affected by property objects, and this data property will be useful to name the class and the data to be included in this ontology because each data will have its name used for identifying it. Then classify all data entered into ontology. Each incoming data must have at least one relationship with other data so that it can be used based on the relationship they have. All data will be given a relationship with each other; after that the data is ready and stored in the form of an OWL file, which will be used later in the application. Next is creating a SPARQL query that will be used to retrieve data from the OWL file. To be able to make the query, PREFIX must first be specified, which is the name of the place of the data [11]. Furthermore, the WHERE is determined, to give a limit on the data to be taken, by determining the conditions or conditions that must be met to retrieve the data. Inside the WHERE, there is a FILTER, which is useful for classifying data retrieval as needed. The next step is to do the making of an application, starting from the design of how the application will look up to the functions in the application. Besides that, it also makes a connection between the OWL file containing the ontology data and the application.

Ontology graphics, or commonly referred to as OntoGraf, is one of the features found in Protégé tools. This feature was introduced to the user, starting from version 4.3 [12]. The Protégé software adapts the Java programming language which can be customized according to user needs [13]. Usually, ontology research

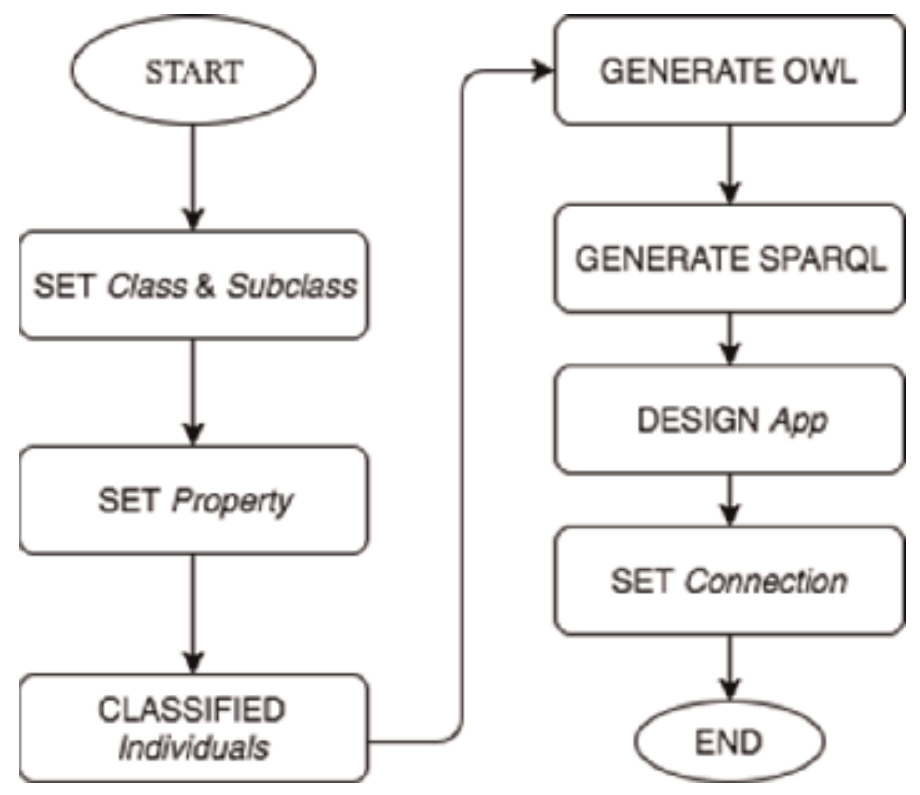

Figure 1.

Flowchart. 
and acquired knowledge using Protégé software. The function of OntoGraf itself is to provide an interface so that users can manage navigation from relationships between classes, properties, and individuals contained in OWL files [14]. By using this menu, the user can see the display of relations between class, property, and individuals in the OWL file. There is an OntoGraf feature that provides interactive support for navigating the OWL ontology relationship. Various layouts are supported to regulate the structure of the ontology [15]. The types of data relationships supported include subclass, individual, domain or range object properties, and equivalence.

Figure $\mathbf{2}$ is an ontograph wherein the Protégé application; there are seven depressive disorders and eight subclasses of depressive disorder. Other specified depressive disorder and unspecified disorder is being one part because they have the same characteristics. The picture shows the depressed mood subclass has two individuals, namely, not experiencing and experiencing. The subclass of psychological diagnosis has two individuals, namely, none and possible; subclass how long describes how long the user has experienced problems such as depressed mood or more irritable, that is, more than 12 months, more than 2 weeks, do not know, more than 1 month, not long ago, and more than 2 years.

Figure 3 is the first-level display of the ontograph. The conclusion is that rice has members, namely, the type of rice that exists. Then as a subclass of rice, there are descriptions and dishes. The description has a brand and variable, while the dishes contain rice dishes. Next level 2 of the ontograph is a member of each subclass. Rice dishes consist of names of processed foods, and brands have members, namely, existing rice brands, while variables have members, namely, the criteria for rice used.

The OWL file is the output generated from the design that has been done before, namely, the design of classes, subclasses, property objects, and datatype properties. The following are the results of the design. The class in the class design is depressive order. Figure 4 shows that the depressive order class has subclasses, which are how long, depressed food, psychological diagnosis, food problems, problems with tempers, menstruation, drugs, and medicinal medicine, while Figure 5 shows the contents of the class and subclass of OWL in the second scenario.

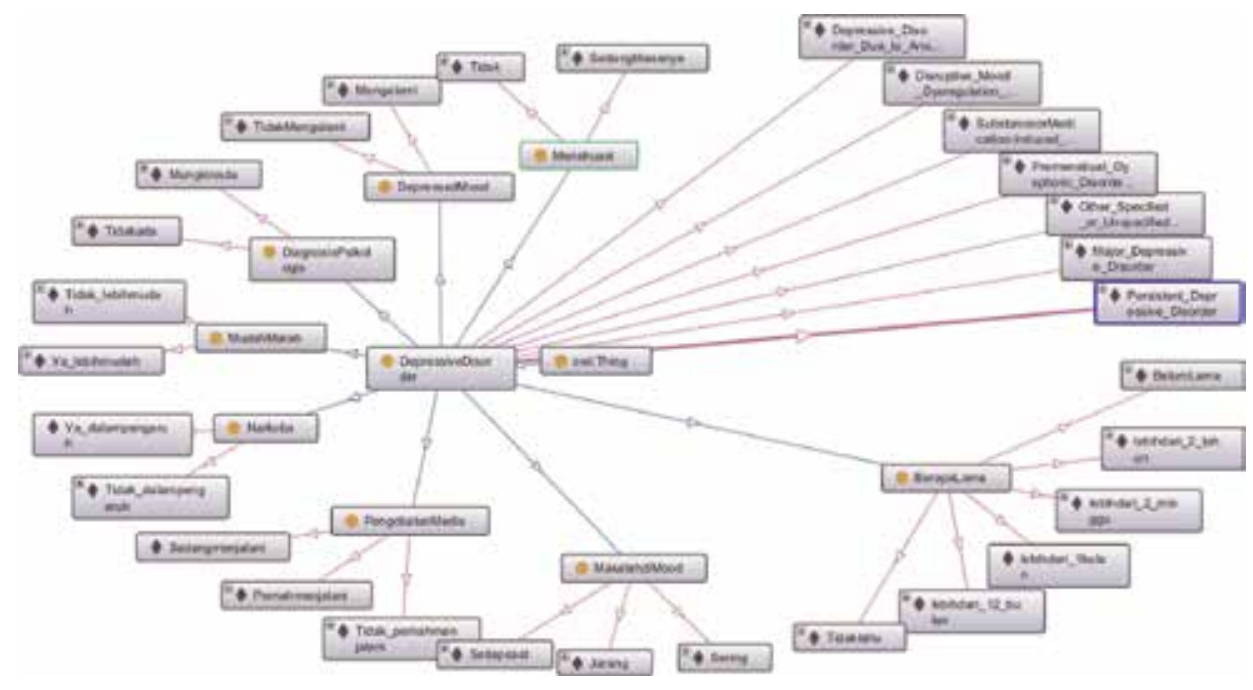

Figure 2.

Ontograph of the first scenario. 
An Ontology-Based Approach to Diagnosis and Classification for an Expert System in Health... DOI: http://dx.doi.org/10.5772/intechopen.88180

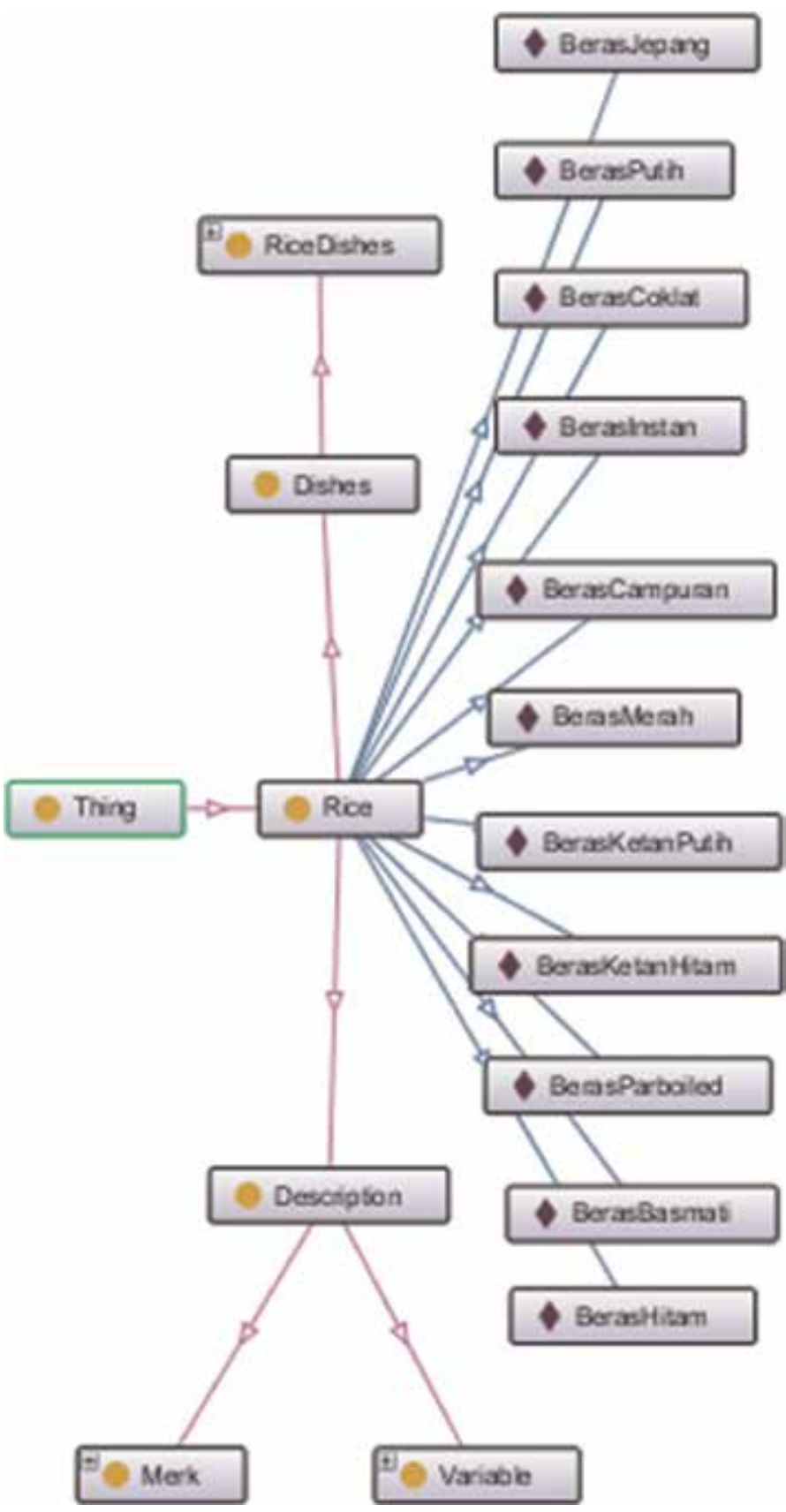

Figure 3.

Ontograph of the second scenario.

The input of the first scenario:

1. How long the user has experienced problems such as depressed mood or more irritable has six individuals, that is, more than 12 months, more than 2 weeks, do not know, more than 1 month, not long ago, and more than 2 years.

2. Depressed mood has two individuals: not experiencing and experiencing. 


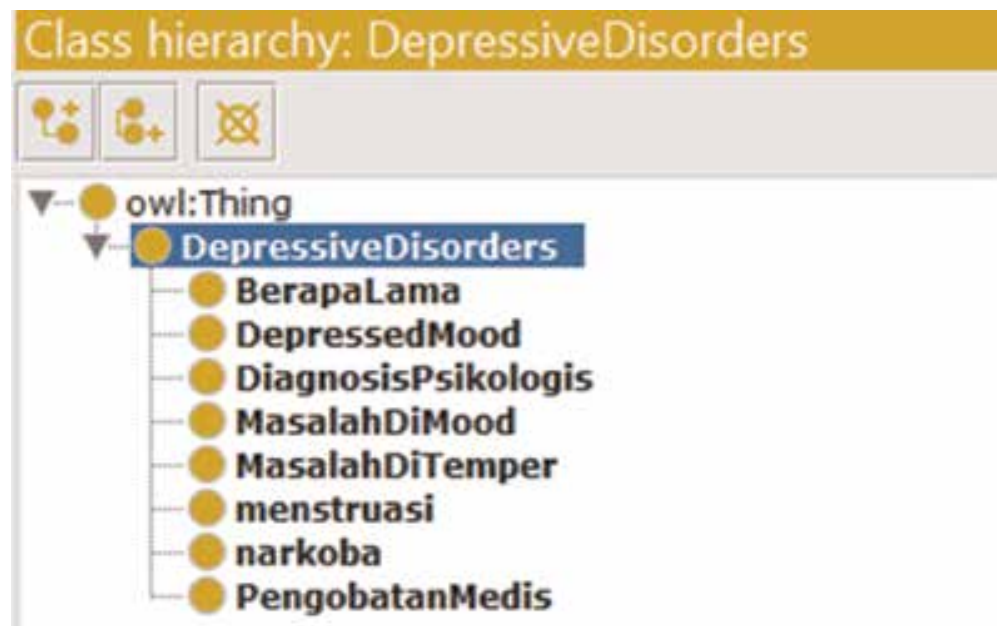

Figure 4 .

Design class of the first scenario.

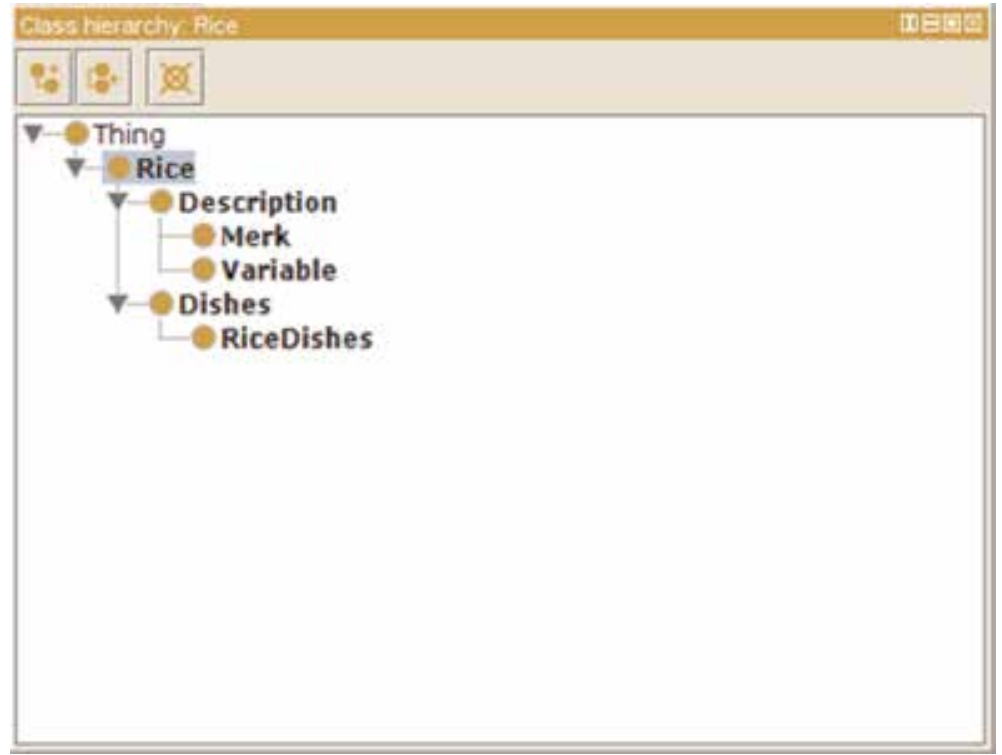

Figure 5 .

Design class of the second scenario.

3. Diagnosis psychology has two individuals: none and may be present.

4. Mood problems have three individuals: all the time, rarely, and often.

5. Easy to get angry has two individuals: yes, it is easier, and no, it is not easier. 
6. Menstruation has two individuals: no and moderate period. This menstruation is only experienced by women, as an initial indication of premenstrual dysphoric disorder.

7. Narcotics have two individuals: users and nonusers.

8. Medical treatment has three individuals: ever undergoing, undergoing, and never undergoing.

The output of the first scenario is the application which can find information about signs of depressive disorder. Users will choose the type of depressive disorder they want to know the information, and then the system will process. Then the desired data will appear; after getting the desired data, the user can try again to find the other data, or if there is nothing to look for, the application is complete.

The input of the second scenario is class rice has property objects and datatype properties that vary according to the characteristics of each rice. Rice class has members, namely, the type of rice. Each type of rice has different attributes. Then brands have types of rice and rice variables. Each subclass rice dish contains rice. These preparations also have object properties and datatype properties that differ according to the needs of each processed rice. Members or identifiers in the rice dish class are types of rice. Subclass brand is a subclass of the brands of rice sold in Indonesia. Each brand has an object property such as compatibility with the type of rice and the characteristic determinant of rice. Subclass variable contains variables that are used to classify the types of rice, and aroma has attributes such as the type of rice and the brand of rice that has these characteristics.

The output of the second scenario is to build an application to make it easy for users to find the most suitable type of rice so that the desired rice processing is appropriate and to find out the application of the ontology method in making expert system applications based on Android.

The next step is to convert the results from Protégé to the database. In the first scenario, we will use a CSV file where the results of Protégé are then exported to a CSV format file with entities containing individuals from depressive disorder and values of properties containing object properties. This step is shown in Figure 6.

Whereas in the second scenario directly using PHP, where the SPARQL query is used to retrieve data from OWL files that have been created using PHP. In this process function filters one and two function as complex character removers so that the results of the OWL can be read clearly [16-18]. This function is essential, so

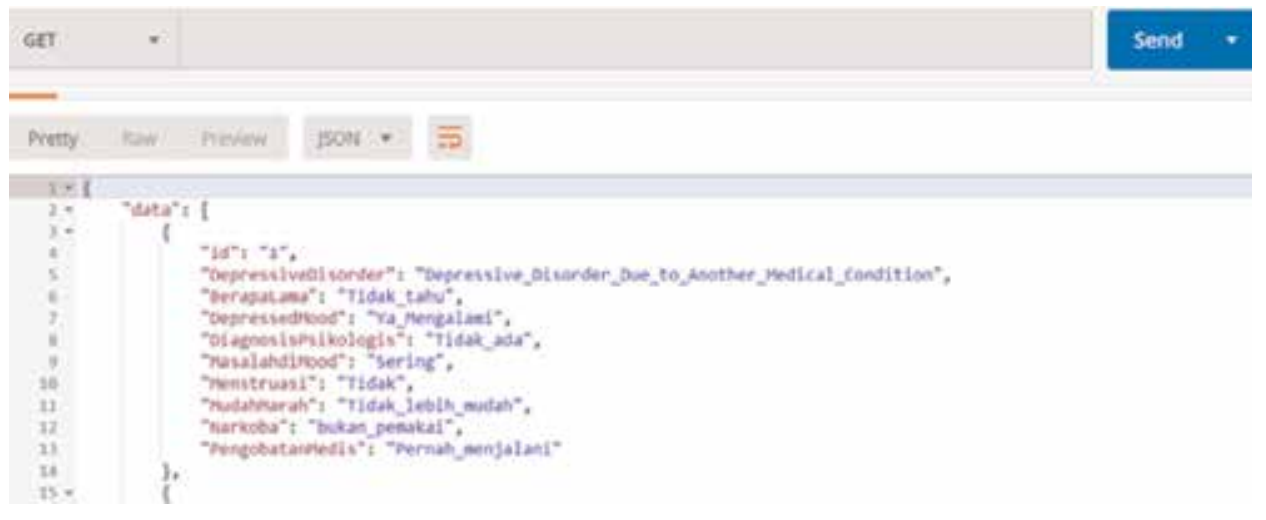

Figure 6.

SPARQL query for the first scenario. 
there is no error in retrieving data from OWL. Also, this function is useful for calling data from the OWL while matching data that has been previously made according to the right results. The match function is given results for the function to

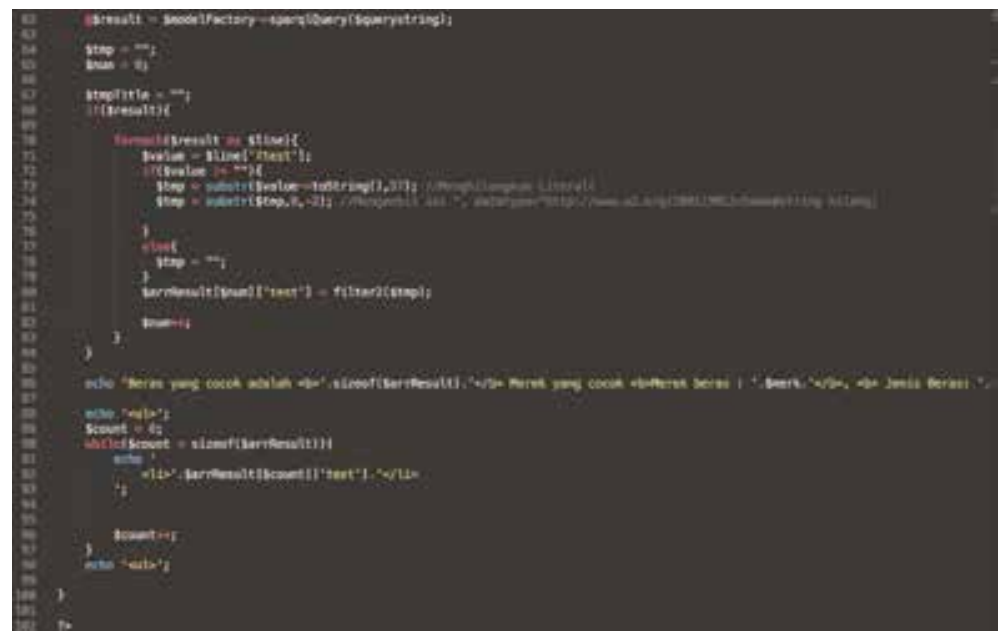

Figure 7.

SPARQL query for the second scenario.
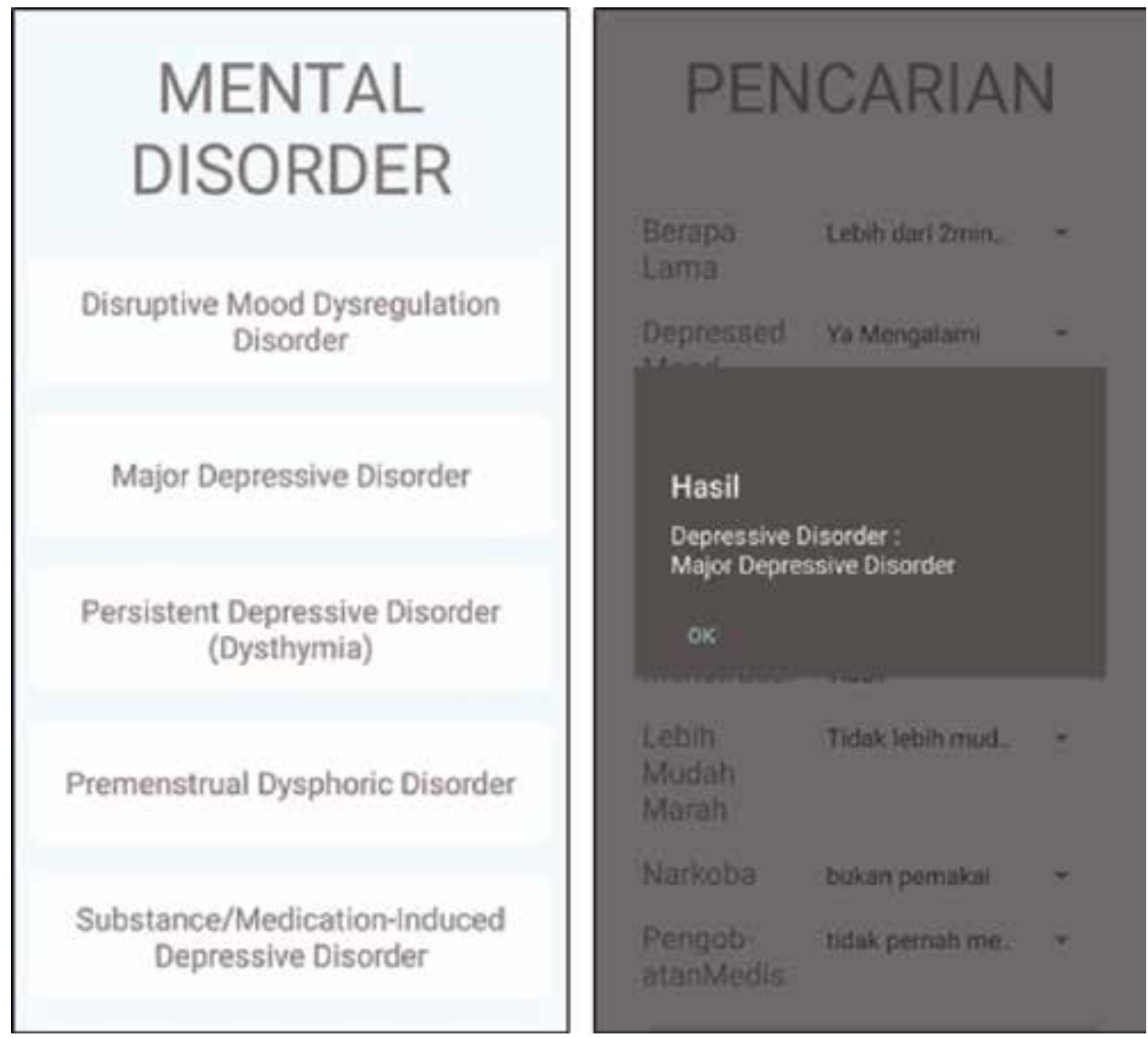

Figure 8.

Result of the first scenario. 
be displayed on the application. Moreover, thus it can be concluded that the query used to retrieve data is SELECT DISTINCT * to retrieve all data using the conditions specified in the WHERE where there are conditions that must be met to choose the right results $[19,20]$. This step is shown in Figure 7.

The following are some of the pseudocodes contained in the design:

\section{Begin}

foreach dishes

if criteria dish_rice $=$ criteria rice

then

rice $=$ rice_variance

end if

After doing the development and the prototype is declared complete, the implementation is done. Implementation is done when publishing the application in Play Store. After that is monitoring the users who are interested in using this application. The application created can be seen on the Google Play Store with the name MentalHelp application for the first scenario and RicheApp for the second scenario.

Figure 8 describes the Disorder search menu, wherein the user can choose the answer that is perceived by the user and then look for what kind of depressive disorder might be suitable, but the user may not find the answer sought. This image is part of the search menu where the results will appear in the selection which matches the existing data and can show the display for depressive info where there is a choice of each type of depressive disorder containing information about each type.

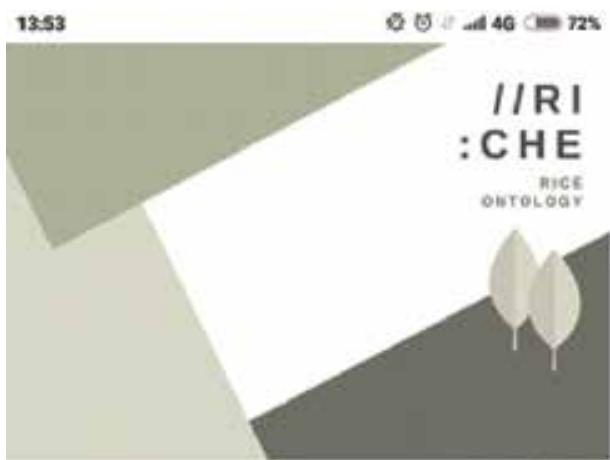

What kind of dish do you want to made? (Choose one of the dishes below that you want to made)

Bakcang

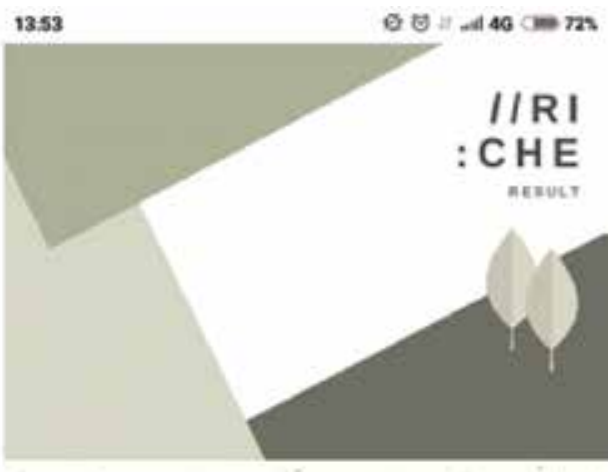

The suitable rice for you to make Kerak Telor is White Rice with example(s) of brand(s) Walet. The seller in Tangerang that sell that rice are Toko Beras. Surya, Toko Beras Raja Ban, dan Toko Beras Wijaya

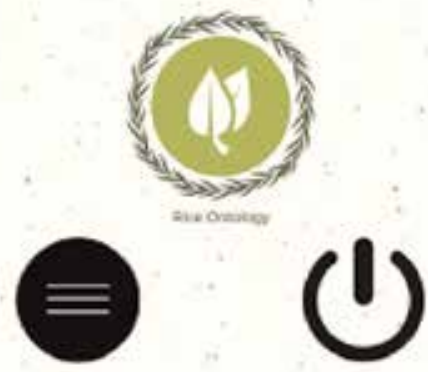

Figure 9.

Result of the second scenario. 
Figure 9 is the main menu display of the application when the application starts. The main menu includes Rice Ontology, Rice Info, Rice Dishes, and Rice Seller. The Rice Selection feature makes it easy to find types of rice processed foods that are common in Indonesia. After the type of processing is selected, the user can click the "Calculate" button. After clicking the "Calculation" button, it will display the type of rice and the brand that sells the rice in Indonesia. There is a menu to return to the selection of processed types, to return to the main menu, or to exit the application.

Based on the results, the information presented in the application is complete and valid following the results of interviews from experts. In the first scenario, several developments exist after the system is created which can make it easier for people to know information about the depressive disorder. Previously to find out the type of depressive disorder, users cannot know quickly, while after this application, users can know that depressive disorder has several types. In the process of estimating the type of depressive disorder, previously it was difficult for the user to estimate whether he had a specific depressive disorder, while after this application, the user could estimate the depressive disorder that he had or whether he did not have it at all.

In the second scenario, previously in the rice selection process, the user used perceptions without specific guidelines and benchmarks, while after the application of the expert system, there could be a proper and correct reference for selecting each rice selection. In the delivery of information, the user previously conveyed information about the suitability of rice with processing based on perceptions and little knowledge of others, while after this application, information about the suitability of rice can be obtained quickly and surely whenever and wherever.

\section{Conclusions}

Based on the results of the research that has been carried out, these are the several conclusions for health and food problems using the ontology method, which produces the following Android-based applications:

In the first scenario: In each type of depressive disorder, it can be concluded that the characteristics of a person experiencing depressive disorder can be seen from how long someone feels it. Besides, it can be seen whether someone becomes more irritable, whether someone always feels sad, whether someone is coming for a month, whether someone has had medical treatment, whether someone is a drug user, and whether someone has another psychological diagnosis. The results of all that can be a feature of a person having a depressive disorder.

In the second scenario: Users have the convenience of being able to choose the type of rice that matches the desired rice processing and recommendations of the right type and brand through an Android-based smartphone application that can be accessed anytime and anywhere. By using this expert system, the process of selecting rice types for beginners becomes more appropriate according to expert recommendations. 
An Ontology-Based Approach to Diagnosis and Classification for an Expert System in Health... DOI: http://dx.doi.org/10.5772/intechopen.88180

\section{Author details}

Friska Natalia*, Dea Cheria and Santi Surya

Universitas Multimedia Nusantara, Tangerang, Indonesia

*Address all correspondence to: friska.natalia@umn.ac.id

\section{IntechOpen}

(C) 2019 The Author(s). Licensee IntechOpen. This chapter is distributed under the terms of the Creative Commons Attribution License (http://creativecommons.org/licenses/ by/3.0), which permits unrestricted use, distribution, and reproduction in any medium, provided the original work is properly cited. (c) BY 


\section{References}

[1] Lee HK, Ko CS, Kim T, Hwang T. Fuzzy ontological knowledge system for improving RFID recognition.

International Journal of Industrial Engineering. 2013;20(1-2):60-71

[2] Chandra D, Ferdinand FN. Ontology system design for herbal plants. International Journal of Latest Trends in Engineering and Technology (IJLTET). 2018;10(3):13-19

[3] Halim FN, Ferdinand CSK. Ontology-based decision support system for hypersensitivity disorder allergy. ICIC Express Letters. 2018;12(8): 847-854

[4] El-sappagh S, Elmogy M. Fuzzy ontology modeling for case base knowledge in diabetes mellitus domain. Engineering Science and Technology an International Journal. 2017;20(3): 1025-1040

[5] Rajendran VV, Swamynathan S. MOSS-IR: Multi-ontology based search system for information retrieval in e-health domain. Procedia Computer Science. 2015;47:179-187

[6] Helmy T, Al-Nazer A, Al-Bukhitan S, Iqbal A. Health, food and user's role ontologies for personalized information retrieval. Procedia Computer Science. 2015;52:1071-1076

[7] Dennis A. System Analysis and Design. 6th ed. America: Wiley Inc; 2014

[8] O’Brien, James A. dan George M. Marakas. Management Information Systems, 10th ed. McGraw-Hill/Irwin, New York, 2011

[9] Kelly Rainer R. Brad Prince. Introduction to Information System. 7th ed. America: Wiley Inc; 2017

[10] Association AP. Diagnostic and Statistical Manual of Mental Disorders, Fifth Edition (DSM-5). America: American Psychiatric Association; 2013
[11] Horridge MA. Practical guide to building OWL ontologies using protege 4 and CO-ODE tools Edition 1.3. 2011

[12] Lee HK, Ferdinand FN, Kim T. Fuzzy ontology-based supply partner matching. ICIC Express Letters: International Journal of Research and Surveys. 2011;5(9B):3329-3334

[13] Xiolong L. Software Engineering and Information Technology. World Scientific; 2015

[14] Upward A, Jones PH. An ontology for strongly sustainable business models: Defining an enterprise framework compatible with natural and social science. Organization \& Environment, Special Issue: Business Models for Sustainability: Entrepreneurship, Innovation, and Transformation (On-Line First). 2015:1-27

[15] Neji H, Bouallegue R. Ontology for mobile phone operating. 2012

[16] Workman M. Semantic Web: Implications for Technologies and Business Practices 1st ed. Springer; 2016

[17] Lee B, James H, Lassila O. The semantic web. Scientic American. 2001: 29-37

[18] Pollock TJ. Semantic Web for Dummies. Indiana: Wiley Publishing Inc.; 2009

[19] Connoly T, Begg C. Database System: A Practical Approach to Design, Implementation, and Management.

America: Publisher Pearson; 2010

[20] Elve AT, Preisig HA. From ontology to executable program code. Computers and Chemical Engineering. 2019;122: 383-394 


\title{
Taxonomy and Ontology Management Tools: A General Explanation
}

\author{
Sukumar Mandal
}

\begin{abstract}
The World Wide Web is the result of a radical new way of thinking about sharing information. This idea seems familiar now, as the web itself has become pervasive. But this radical new way of thinking has even more profound ramifications when it is applied to a web of data like the semantic web. These ramifications have driven many of the design decisions for the semantic web standards and have a strong influence on the craft of producing quality semantic web applications. Until several years ago, the semantic web was primarily in a research phase. The new implementations of it were mainly to demonstrate the potential of the idea. While much of the activity related to semantic technology still takes place within the academic community, there are now real-world examples of the technology to use as a model. This paper has selected the matured level open-source tools for management of taxonomy and ontology in semantic web environment. Apart from this it also presents some important snapshot available in an online environment for designing and developing the taxonomy and ontology. This is very helpful to the users in using the concept of science and technology in cloud computing and online and offline environment.
\end{abstract}

Keywords: ontology, semantic web, TemaTres, visual vocabulary, and taxonomy

\section{Introduction}

This is the age of technology where digital information resources are increasing. It is a good concept for creating both the present and next-level automated and digital library system because it can highly be performed in the semantic web environment for executing the functional activities of libraries and any information resource centers. It helps the researchers for reviewing their literature. Here users can easily access and understand the terms and their relation from these interfaces for the better management of library and information services. It manages the big data in semantic web-linked data environment. It is also possible to manage the bibliographic link data in the visual vocabulary format, so the users can easily download the content as well as information. This is a web-based architecture in the Internet. Semantic web is one of the important aspects in cloud computing [1]. A lot of linking of web resources are available in an online environment [2]. The idea of a web of information was once a technical idea accessible only to a highly trained elite of information professionals: IT administrators, librarians, information architects, and other resources [3]. It is known as big data concept in semantic web level. It is based on open-source standards and formats including SPARQL, RDF, JSON, HTML, 
XHTML, DTD, METS, MODS, etc. for managing the different web resources in any institutions or in any library [4]. These formats help to draw the visual graph of each uniform resource identifier for maintaining and managing the big data in the Internet [5]. Visual vocabulary is also an important concept in semantic web because it stores the large number of data and their linking also [6]. It is possible to manage the relations of different subjects by their specific identities, properties, and entities [7]. Big data is easily managed by using the open-source software and open standard formats in an online environment [8]. Users can easily access their necessary information by using this semantic web software in an Internet environment because it can manage the controlled vocabularies and their relationships of each element of any subject fields [9]. So, obviously it can save the time of reader and library professionals also [10]. It can manage the N-Triples, a format for storing and transmitting data, and Terse RDF Triple Language (Turtle), and XML provides an elemental syntax for content structure within documents yet associates no semantics with the meaning of the content contained within [11]. XML is not at present a necessary component of semantic web technologies in most cases, as alternative syntaxes exist, such as Turtle [12]. The World Wide Web contains many billions of pages. The SNOMED CT medical terminology ontology alone contains 370,000 class names, and existing technology has not yet been able to eliminate all semantically duplicated terms [13]. Any automated reasoning system will have to deal with truly huge inputs [14]. The objective of this chapter is very simple to highlight some popular taxonomy, ontology, and visual vocabulary through open-source tools for the users as well as library professionals. The important tools have been discussed in the next section. It also shows how it works in the web environment.

\section{Objectives}

The essential objectives of this study are explained as below:

i. To explore the modern tools and techniques those are available in an online digital environment for easy generation of taxonomy and ontology in different aspects.

ii. To highlights and visualize some terms by using these open source tool.

iii. To show the taxonomy concepts in an academic and research environment of different relations both technically and graphically.

iv. To design an integrated framework in an offline and online environment by using the open source tool and technique for each an every concept and their relations for the management of information resources.

\section{Methodology}

The process and methods of this study are one of the important aspects for designing and creating taxonomy and ontology. In this original research paper, the matured level open-source tools and techniques have been selected for the creation of visual vocabulary. Apart from this it also explores how to construct a visual thesaurus in an offline and online environment on Ubuntu operating system for easy constructing of the different terms and relations such as narrower term, broader term, related term, used for, scope note, and so on. So, this paper consists of two parts such as online and offline for term relations. 


\section{Visuwords}

Visuwords is an online visual graphical dictionary as well as controlled vocabulary tool. Actually it provides the visual interface of each word and its respective meaning on the basis of English grammar. This is very user-friendly for definition of any words from any subjects can be represented in a separate color dashboard. Wheel and zoom are possible by using the mouse. It explores the word synonym, derivation, and antonym to extend the meaning of any facets available in an web-based environment (https://visuwords.com/library). Figure 1 represents the Visuwords interface in an online environment for visual graphic dictionary. It is possible to easily identify the noun, verb, adjective, and adverb for construction the thesaurus against in a word.

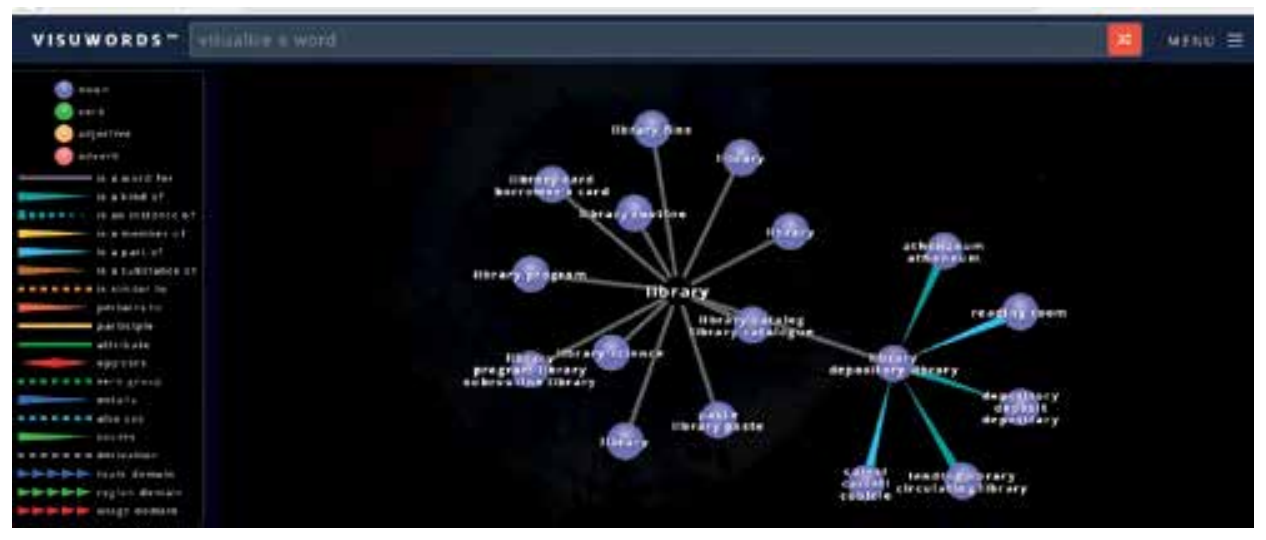

Figure 1.

Visuwords interface for visual graphic dictionary (https://visuwords.com/library).

\section{Linked open vocabularies}

Linked Open Vocabularies is a new concept in semantic web ontology. It consists of large amount of dataset around 660 vocabularies as on March 10, 2019. It fully supports the OWL and RDF framework for better management and identification of the right link available in an online database. SPARQL endpoint is easily accessed by using this interface. Properties and classes can be done on the basis of ontology concept that is subject, predicate, and object (https://lov.linkeddata.es/dataset/ lov/). Here all the dataset belongs to the vocabulary content or element type. Figure 2 represents the Linked Open Vocabularies interface in semantic web for different elements and their relationship.

\section{WebVOWL}

WebVOWL is a web-based ontology visualization open-source tool and is very interactive. This is written by high-level programming language named as Java which implements the visual notation and graphical interface of different nodal points in web ontology language. It explores the creation of ontology and combined forced graph in different formats such as JSON, RDF, and XML and other relevant file formats in an integrated semantic web environment (http://visualdataweb.de/ webvowl/). Figure 3 represents the WebVOWL interface in semantic web for linking a custom ontology based on URL and URI. 


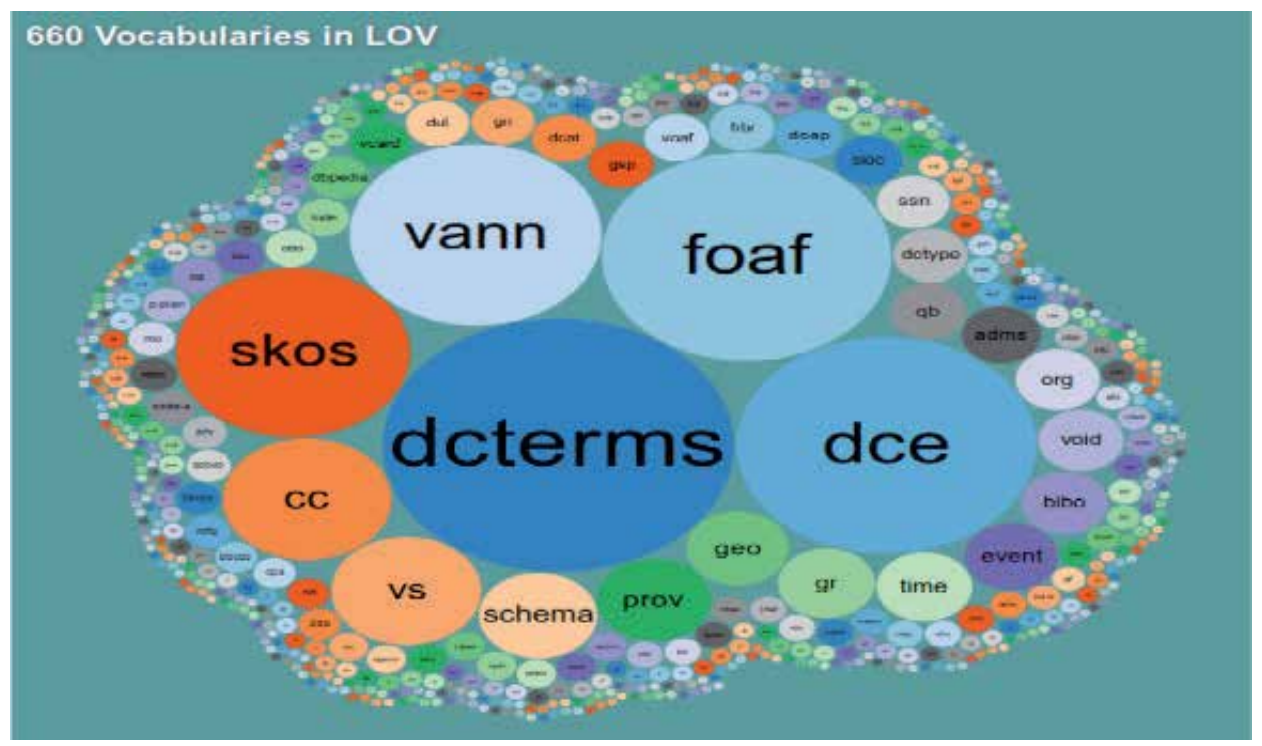

Figure 2.

Linked open vocabularies interface in semantic web ontology (https://lov.linkeddata.es/dataset/lov/).

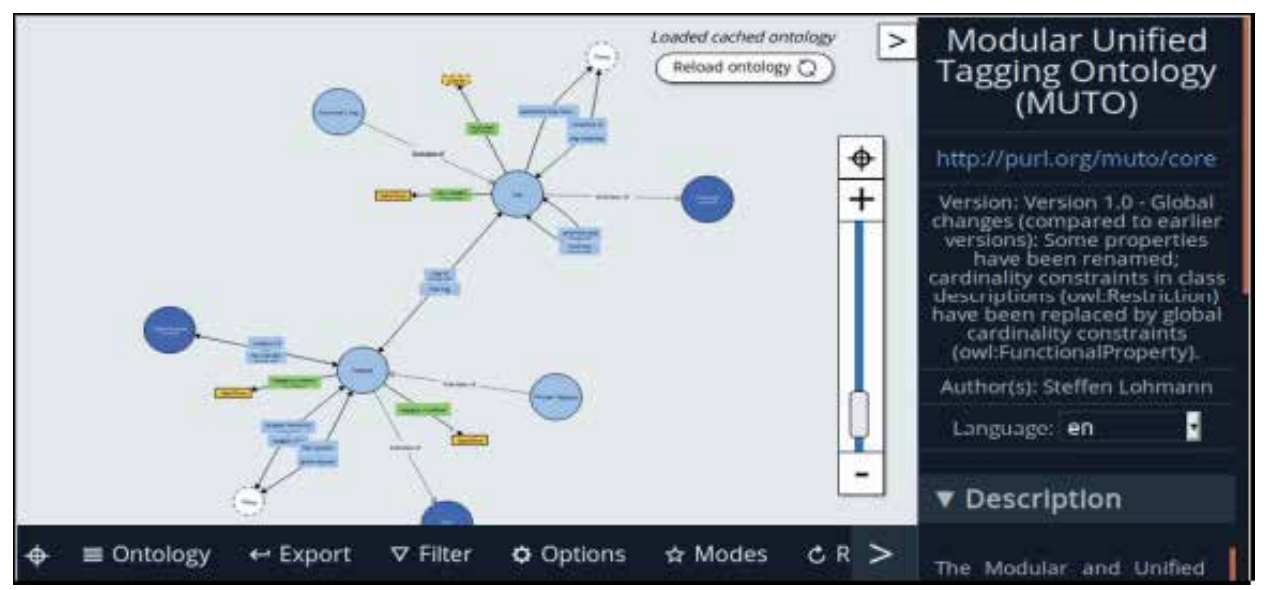

Figure 3.

WebVOWL interface in semantic environment (http://visualdataweb.de/webvowl/).

\section{NavigOWL}

NavigOWL is a graph visualization open-source Java-based tool in web ontology environment. This tool is a very interactive high-performance graph layout in semantic web environment. Here all ontologies are structured based which facilitate the patrons in thinking the mental map in an ontology environment. Protégé can be graphically represented by using this plug-in for better management of link and their elements (http://home.deib.polimi.it/hussain/navigowl/index. html). Figure 4 represents the NavigOWL interface in web ontology environment. It loads and uploads the RDF/OWL ontology file for creation of graph and taxonomy in different nodes and edges. 


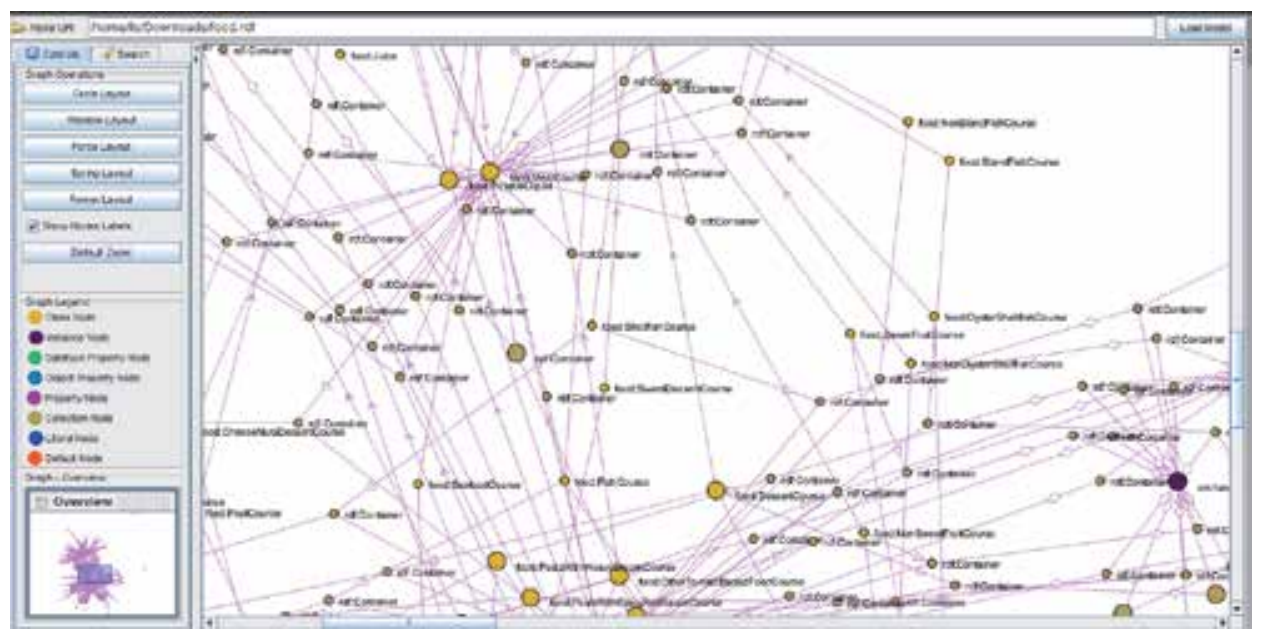

Figure 4.

NavigOWL interface in web ontology.

\section{Unilexicon vocabulary}

Unilexicon is a visual online controlled vocabulary and thesaurus construction server. This is a high-level open-source software for designing taxonomy and classification. It fully supports the faceted navigation and tagging for document and knowledge classification on the basis of simple knowledge organization system (SKOS). Institutional digital repository can easily be designed and developed by using the metadata and semantic search tool. This is also known as record management tool (https://unilexicon.com/). Figure 5 represents the Unilexicon vocabulary interface. This is independent of content management system by using tag and facet count concept.
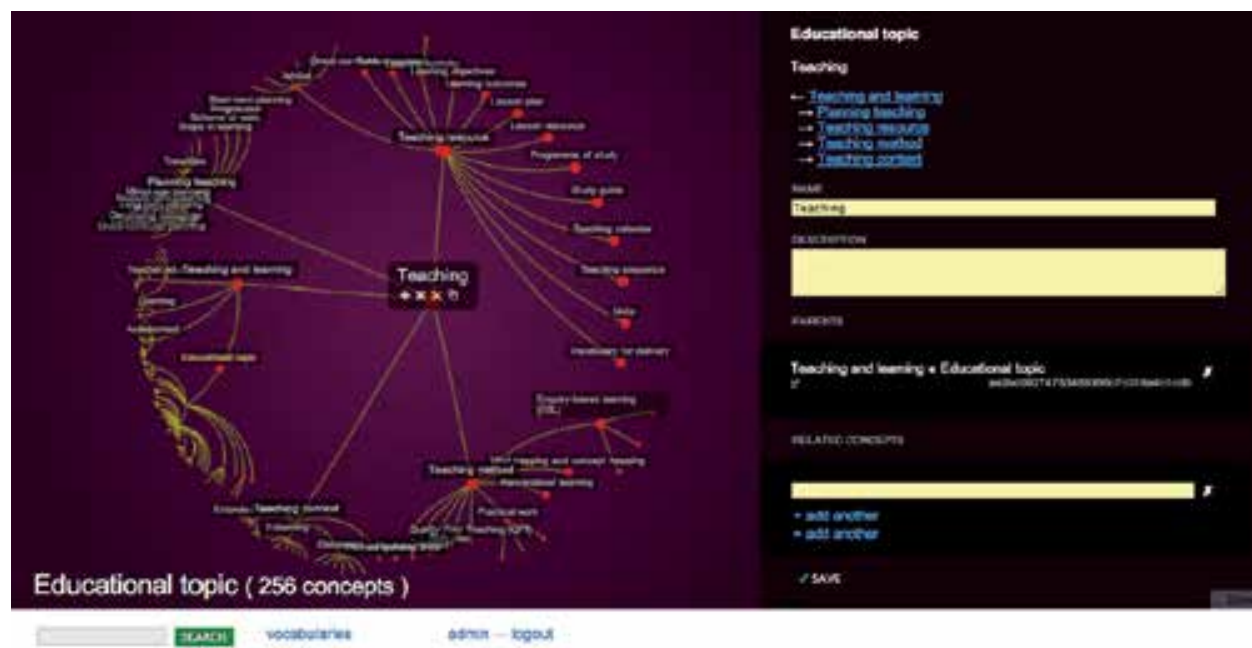

asmen - vegost

Figure 5.

Unilexicon vocabulary interface in taxonomy (https://unilexicon.com/). 


\section{Visual vocabulary TemaTres}

TemaTres is a web-enabled open-source thesaurus construction software and written in PHP programming language. It is also known as vocabulary server for management formal representations of knowledge, thesauri, taxonomies, and multilingual vocabularies [15]. It has many features including SPARQL Protocol and RDF Query Language, Meta-terms (define facets, collections or arrays of terms), support for multilingual thesaurus, expose vocabularies with powerful web services, search terms suggestion, display terms in multiple deep levels in the same screen, search expansion, vocabulary harmonization, relationship between terms (BT/NT, USE/UF, RT), no limits to number of terms, alternative labels, levels of hierarchy, etc., systematic or alphabetic navigation, complete export in XML format (Zthes, TopicMaps, MADS, Dublin Core,VDEX, BS 8723, SiteMap, SQL), complete export in RDF format, complete export in txt, scope notes, historical and bibliographical notes, user management, terms and user supervision, duplicates terms control, free terms control, quality assurance functions (Duplicates and free terms, ilegal relations), multilingual interface, easy install, utility to import thesauri from tabulated textfiles, unique code for each term, terminology mapping with multilingual, term reports for editors, workflow like candidate, accepted and rejected terms, allow to create user-defined relationships, allow to define published and hidden labels, relationships between terms and web entities, export to WXP (WordPress XML) and import and export data in Skos-core (Figure 6).

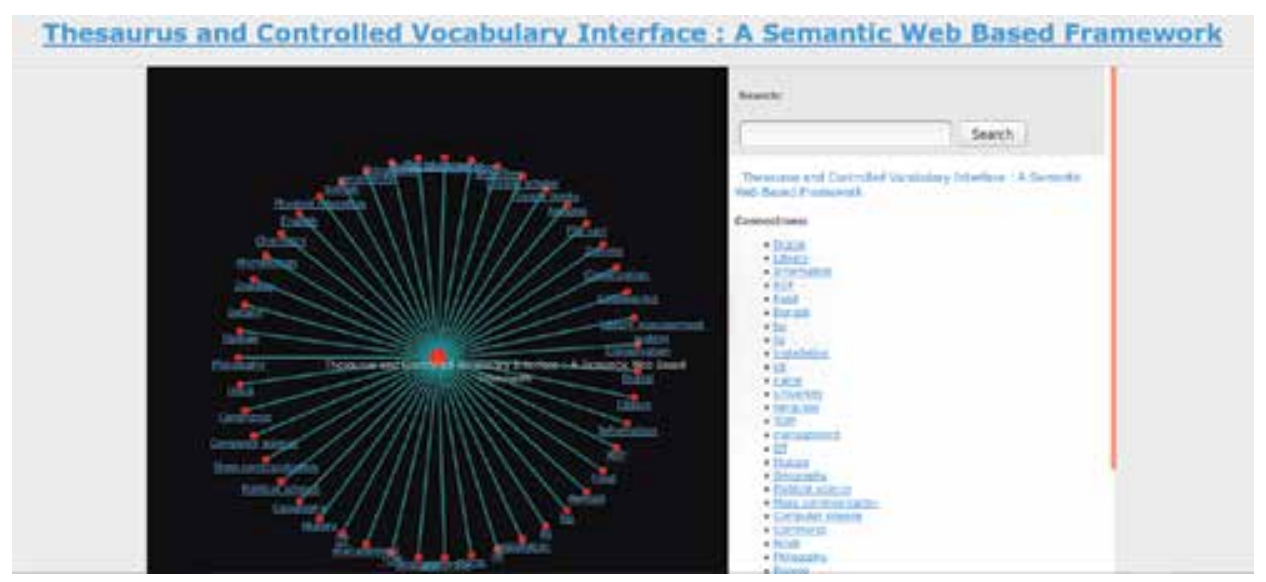

Figure 6.

Visual vocabulary interface in TemaTres.

\section{Conclusion}

Vocabulary creation is important for the users in any library including academic, public, and research libraries. It can manage and maintain the narrower terms and broader terms in respect to one term of a specific subject like philosophy, Bengali, history, chemistry, etc. Search interfaces of visual vocabulary are very sophisticated, and here quick search facilities are available also. The alphabetical index part contains each and every term, including synonyms, quasi-synonyms, and antonyms, occurring in the systematic part, along with its narrower terms. It controls the different terms used in indexing, providing a means of translating the natural language authors, indexers, and enquirers into a more constrained language used for indexing and retrieval. Visual vocabulary is one of the important concepts 
of formulation of different words and their associated terms. All the terms and correlated terms against one specific subject are to be appeared in a visual vocabulary interface. It can manage the different terms including broader terms, narrower terms, and both preferred and non-preferred terms. In this section overview, the concept of a visual vocabulary is a strategy that draws inspiration from the text retrieval community and enables efficient indexing for different terms of a specific item types. Since the occurrence of a given word tends to be sparse across different documents, an index that maps words to the files in which they occur can take a keyword query and immediately produce relevant content. Obviously, it can conclude that the above tools highly overview the taxonomy and ontology in semantic web environment for easy access and download of metadata as well as full-text resources and their measures by graph. Thesaurus, taxonomy, and ontology are properly managed by using these tools for better management and retrieval of linked and resources in the web environment.

\section{Author details}

Sukumar Mandal

Department of Library and Information Science, The University of Burdwan, Golapbag, West Bengal, India

*Address all correspondence to: sukumar.mandal5@gmail.com

\section{IntechOpen}

(C) 2019 The Author(s). Licensee IntechOpen. This chapter is distributed under the terms of the Creative Commons Attribution License (http://creativecommons.org/licenses/ by/3.0), which permits unrestricted use, distribution, and reproduction in any medium, provided the original work is properly cited. (cc) BY 


\section{References}

[1] Alfaries A, Bell D, Lycett M. Motivating service re-use with a web service ontology learning. International Journal of Web Information Systems. 2013;9(3):219-241. DOI: 10.1108/IJWIS-12-2012-0035

[2] Benslimane SM, Malki M, Bouchiha D. Maintaining web application: An ontology-based reverse engineering approach. International Journal of Web Information Systems. 2009;5(4):495-517. DOI: $10.1108 / 17440080911006225$

[3] Bygstad B, Ghinea G, Klæboe G-T. Organisational challenges of the semantic web in digital libraries: A Norwegian case study. Online Information Review. 2009;33(5):973985. DOI: $10.1108 / 14684520911001945$

[4] Calaresu M, Shiri A. Understanding semantic web: A conceptual model. Library Review. 2015;64(1/2):82-100. DOI: 10.1108/LR-09-2014-0097

[5] Chi Y-L, Chen H-C. Ontology and semantic rules in document dispatching. The Electronic Library. 2009;27(4):694707. DOI: $10.1108 / 02640470910979633$

[6] Esserhrouchni OEI, Frikh B, Ouhbi B, Ibrahim IK. Learning domain taxonomies: The TaxoLine approach. International Journal of Web Information Systems. 2017;13(3):281301. DOI: 10.1108/IJWIS-04-2017-0024

[7] Khan SA, Bhatti R. Semantic web and ontology-based applications for digital libraries: An investigation from LIS professionals in Pakistan. The Electronic Library. 2018;36(5):826-841. DOI: 10.1108/EL-08-2017-0168

[8] Ko YM, Song MS, Lee SJ. Construction of the structural definition-based terminology ontology system and semantic search evaluation. Library Hi Tech. 2016;34(4):705-732. DOI: 10.1108/LHT-08-2016-0090
[9] Lausen H, Ding Y, Stollberg M, Fensel D, Hernández RL, Han S-K. Semantic web portals: State-ofthe-art survey. Journal of Knowledge Management. 2005;9(5):40-49. DOI: $10.1108 / 13673270510622447$

[10] Llanes-Padrón D, Pastor-Sánchez J-A. Records in contexts: The road of archives to semantic interoperability. Program. 2017;51(4):387-405. DOI: 10.1108/PROG-03-2017-0021

[11] Navarro-Galindo JL, Samos J. The FLERSA tool: Adding semantics to a web content management system. International Journal of Web Information Systems. 2012;8(1):73-126. DOI: $10.1108 / 17440081211222609$

[12] Nguyen H-M, Nguyen H-Q, Tran K-N, Vo X-V. GeTFIRST: Ontologybased keyword search towards semantic disambiguation. International Journal of Web Information Systems. 2015;11(4):442-467. DOI: 10.1108/ IJWIS-06-2015-0019

[13] Nguyen Q-M, Cao T-D. A novel approach for automatic extraction of semantic data about football transfer in sport news. International Journal of Pervasive Computing and Communications. 2015;11(2):233-252. DOI: 10.1108/IJPCC-03-2015-0018

[14] Salvadori IL, Huf A, Oliveira BCN, Mello RS, Siqueira F. Improving entity linking with ontology alignment for semantic microservices composition. International Journal of Web Information Systems. 2017;13(3): 302-323. DOI: 10.1108/

IJWIS-04-2017-0029

[15] Mandal S. Developing thesaurus construction through Tematres for the college libraries under the University of Burdwan. International Journal of English Language, Literature in Humanities. 2016;4(6):302-316 



\section{Edited by Andino Maseleno and Marini Othman}

Advance exact science of nowadays inherited the original ontology's its efforts to systemize and conceptualize. In this respect the concept of ontology is now a nonspeculative methodology for both studying reality objects and its used tools, which both instruments are important for our orientation in the space of physical, technical, mental and societal world. So, within the exactly considered ontology, we deal with the process

of studying, as well as with the outcome of such studying of the objects observed or created by man and their respective made by man concepts, relations between them and relations between their systems in the fields of the given scientific branches.

This book Ontological Analyses in Science, Technology and Informatics - the second volume released within the framework of the IntechOpen project in this field - is the illustration of application of the concept of ontology approach understood in the modern and exact way, it is the presentation of the Ontology science. This book covers the examples of the modern ontology approach, especially the approach in the branch of the Information Science dealing with the Inference and Proof, Knowledge patterns, CrossApplication Communication, Diagnosis and Expert systems in Health and Food and the Taxonomy problems. The intended readers of this book are researchers, students and all the practitioners in the field.

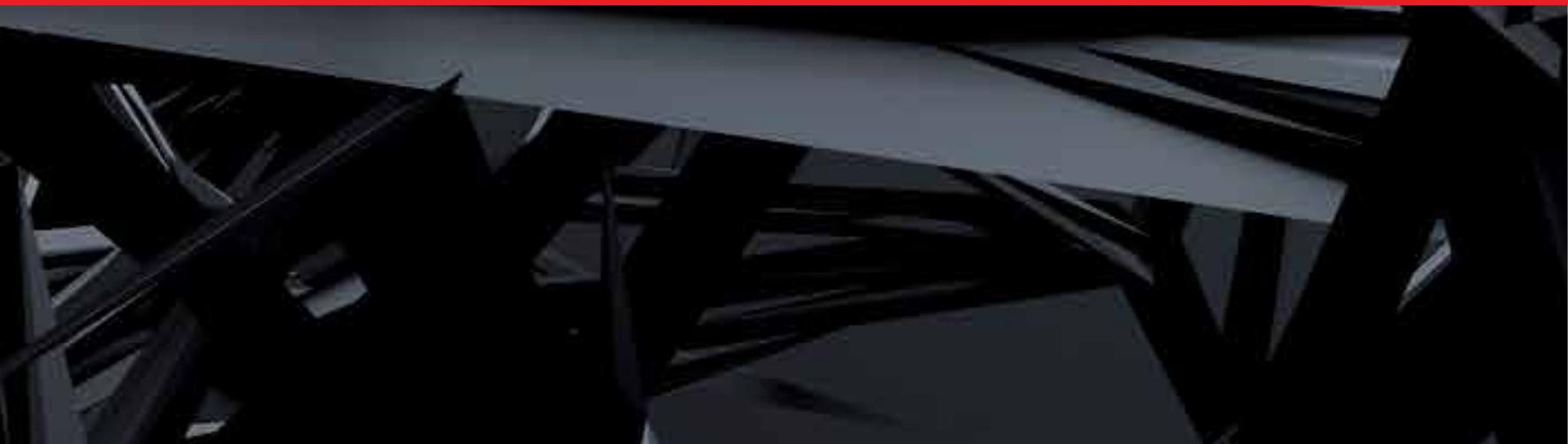

